\title{
A!
}

This is an electronic reprint of the original article.

This reprint may differ from the original in pagination and typographic detail.

Puska, Martti; Nieminen, Risto

\section{Theory of Positrons in Solids and on Solid Surfaces}

Published in:

Reviews of Modern Physics

DOI:

10.1103/RevModPhys.66.841

Published: 01/07/1994

Document Version

Publisher's PDF, also known as Version of record

Please cite the original version:

Puska, M., \& Nieminen, R. (1994). Theory of Positrons in Solids and on Solid Surfaces. Reviews of Modern Physics, 66(3), 841-897. https://doi.org/10.1103/RevModPhys.66.841

This material is protected by copyright and other intellectual property rights, and duplication or sale of all or part of any of the repository collections is not permitted, except that material may be duplicated by you for your research use or educational purposes in electronic or print form. You must obtain permission for any other use. Electronic or print copies may not be offered, whether for sale or otherwise to anyone who is not an authorised user. 


\title{
Theory of positrons in solids and on solid surfaces
}

\author{
M. J. Puska and R. M. Nieminen \\ Laboratory of Physics, Helsinki University of Technology, 02150 Espoo, Finland
}

Various experimental methods based on positron annihilation have evolved into important tools for researching the structure and properties of condensed matter. In particular, positron techniques are useful for the investigation of defects in solids and for the investigation of solid surfaces. Experimental methods need a comprehensive theory for a deep, quantitative understanding of the results. In the case of positron annihilation, the relevant theory includes models needed to describe the positron states as well as the different interaction processes in matter. In this review the present status of the theory of positrons in solids and on solid surfaces is given. The review consists of three main parts describing (a) the interaction processes, (b) the theory and methods for calculating positron states, and (c) selected recent results of positron studies of condensed matter.

\section{CONTENTS}

I. Introduction

II. Positron-Solid Interaction

A. Before thermal equilibrium

1. High-energy region

2. Low-energy region

B. During thermal equilibrium

1. Positron diffusion equation

2. Positron diffusion coefficient

C. Positron trapping into defects

1. Positron trapping model

2. Positron trapping coefficient

3. Resonance trapping

4. Positron trapping in semiconductors

5. Positron trapping at voids in metals

6. Positron trapping at surfaces

III. Positron States in Solids: Models

A. Two-component density-functional theory

1. Generalized Kohn-Sham method

2. Local-density approximation

3. Delocalized positron states

4. Localized positron states

B. Positron states in semiconductors and insulators

C. Positron states on solid surfaces

D. Methods for positron states in solids

E. The momentum distribution of electron-positron pairs

IV. Positron States in Solids: Results and Discussion

A. Delocalized positron states in perfect solids

1. Positron and electron energy levels in solids

2. Positron deformation potential

3. Positron bulk lifetimes

B. Localized positron states at vacancy-type defects in solids

1. Results of two-component density-functional theory: vacancies in metals

2. Beyond the local-density approximation: Clean vacancies and vacancy clusters

3. Vacancies and vacancy clusters decorated with impurities

4. Rare-gas bubbles in metals

5. Defects in semiconductors: electronic and ionic structures

6. Defects in semiconductors: positron states

C. Positron states on solid surfaces

1. Properties of the positron surface state

2. Positron-annihilation-induced Auger spectroscopy
D. Exotic systems

1. High- $T_{c}$ superconductors

2. Fullerenes and related materials $\quad 889$

V. Final Remarks

Acknowledgments

892

893

References

893

\section{INTRODUCTION}

During the last two decades experimental techniques based on positron annihilation were established among the important methods for probing the electronic and atomic structure of solids (see Hautojärvi, 1979; Brandt and Dupasquier, 1983; Schultz and Lynn, 1988; Ishii, 1992; Dupasquier, 1993; ICPA85, ICPA88, ICPA91, SLO90, SLO92). Much as in the case of other methods, the theory underlying positron annihilation has developed from simple models describing the positronsolid interaction to "first-principles" methods predicting the annihilation characteristics for different environments and conditions. This development has paralleled the development of electronic structure calculations, which in turn has leaned heavily on the progress in computational techniques. The conceptual basis of electronic structure calculations lies in density-functional theory (for reviews, see Lundqvist and March, 1983 and Jones and Gunnarsson, 1989), and this theory can be generalized to include the positron states (Nieminen et al., 1985; Boroński and Nieminen, 1986). The density-functional theory itself has been the subject of many investigations. Essential is the approximation of the so-called exchangecorrelation energy. The most important practical approximation is the local-density approximation (LDA). It constitutes the basis of most positron-state calculations as well.

A brief history of an annihilating positron is as follows. After its introduction into the solid, the energetic positron loses energy in the interactions with the material. With decreasing energy, the interactions scan various ionization processes, creation of electron-hole pairs, and positron-phonon interactions. Thereafter the positron lives in thermal equilibrium with the environment, and 
its state develops in real space as a diffusion process, in which the positron interactions with phonons are quasielastic; i.e., the positron momentum distribution is conserved. During diffusion the positron interacts with defects in the solid, and trapping of the positron into a localized state may happen. Eventually, the positron annihilates an electron, resulting in the emission of gamma rays, which convey the experimental information.

The thermalization and diffusion processes can be described as the evolution of the positron distribution function in the momentum and real spaces. The distribution function can be obtained in principle, by solving the Boltzmann equation, which includes the drift effects due to the distribution gradients and possible external fields as well as the effects due to various types of scattering. The Monte Carlo methods suit the simulation of the early stages of the slowing-down process. In this region the approach using the Boltzmann equation is hindered by the narrowness of the distribution functions. The evolution of the real-space positron distribution after thermali- zation is conventionally described by the diffusion equation, for which the source term, the implantation profile, is generated by, for example, the Monte Carlo method. The character of the trapping process depends on the competition between transport and capture. Its theoretical analysis requires the solving of the diffusion problem and/or the calculation of the transition rates, using, for example, Fermi's golden rule.

The description of the state of a thermalized positron in a perfect bulk crystal or that of a positron trapped at a defect requires the solution of the Schrödinger equation. As a matter of fact, the first requirement for a valid theory is that it give a realistic description of the positron distribution and energetics in the solid. Apart from its depletion near the repulsive ion cores, a nearly uniform density can be a starting approximation for the positron in a perfect metal lattice. However, the crystal structures of materials with covalent bonds usually contain an alternation of open and atom-bond regions, leading to a very nonuniform positron distribution. This can be clearly

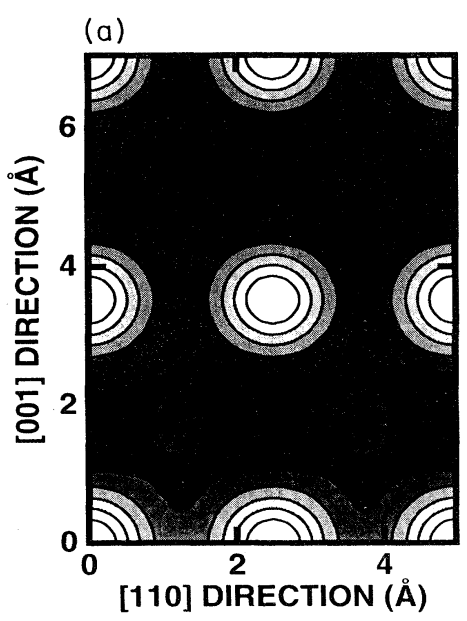

(c) GRAPHITE

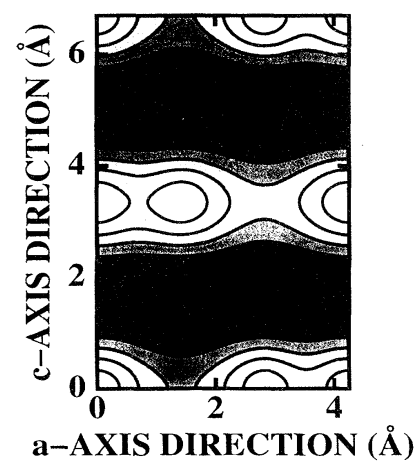

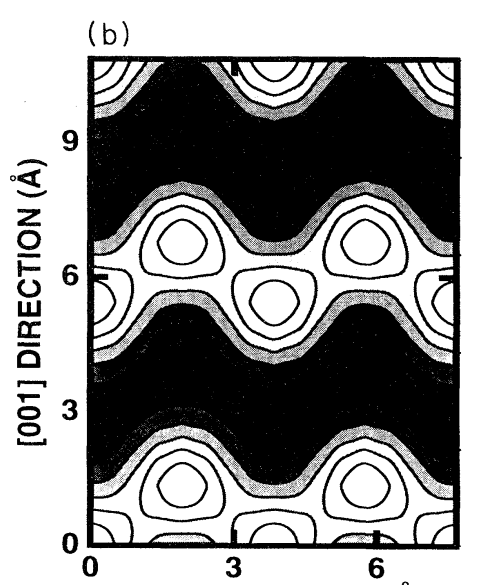

[110] DIRECTION (Å)

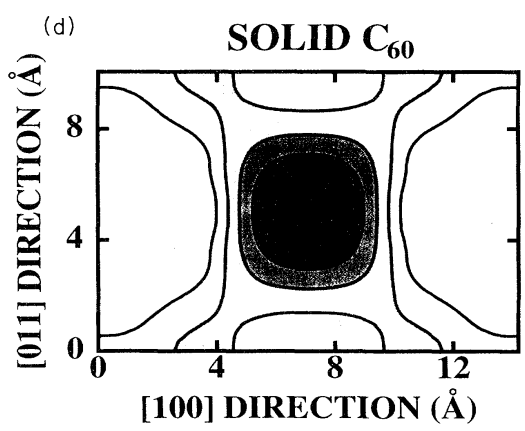

FIG. 1. Positron wave functions in perfect solids: (a) fcc Ni; (b) Si with diamond structure; (c) graphite; (d) solid $\mathrm{C}_{60}$. The calculations were performed using the superimposed-atom method (Puska and Nieminen, 1983a). The contour spacing is one-sixth of the maximum value. The wave function is vanishingly small inside the ion-core regions and inside the $\mathrm{C}_{60}$ molecules, which show in white in the figure. The darker shading indicates larger values of the wave function. 


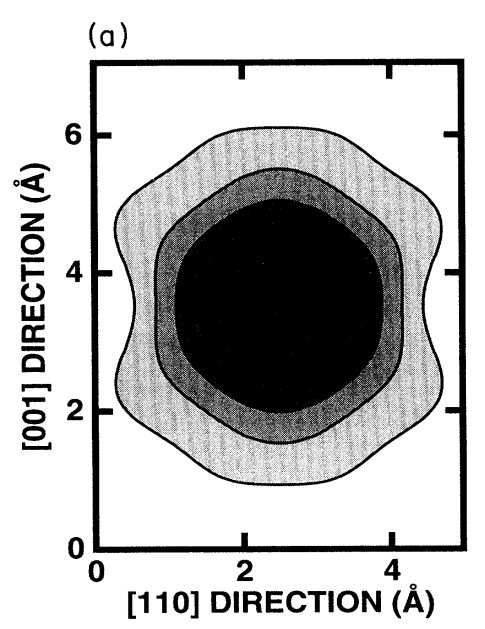

(b)
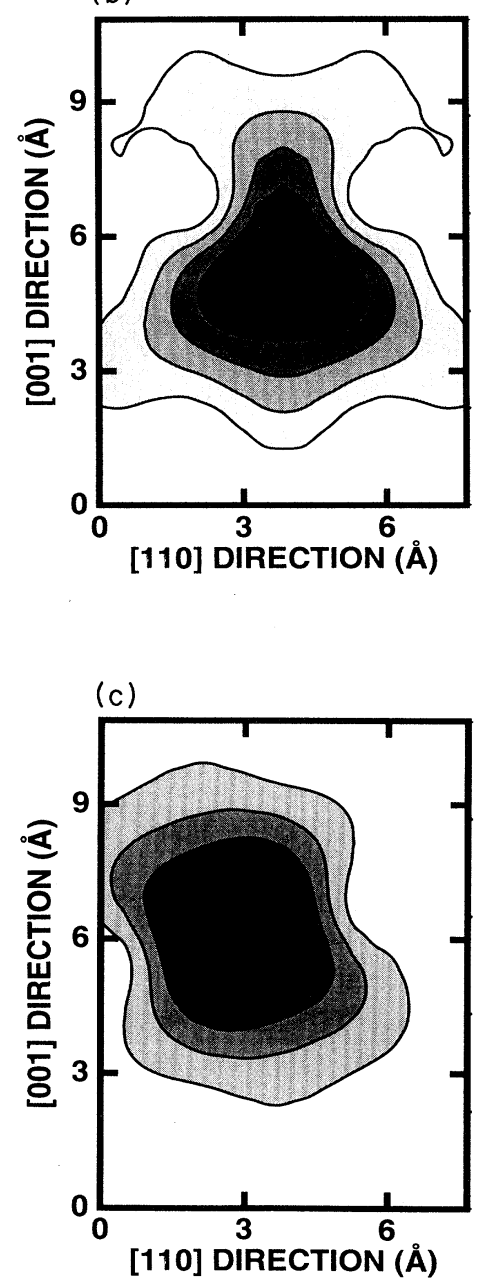

FIG. 2. Positron wave functions for vacancy-type defects in solids: (a) vacancy in fcc $\mathrm{Ni}$; (b) vacancy in $\mathrm{Si}$ with diamond structure; (c) divacancy in $\mathrm{Si}$. The calculations were performed using the superimposed-atom method (Puska and Nieminen, 1983a). See the caption of Fig. 1. The figure planes correspond to those for the perfect crystals in Figs. 1(a) and 1(b). seen from Fig. 1, which shows the calculated positron wave functions in a few representative cases for perfect lattices. At open-volume defects in solids, there exist localized bound states for positrons. As shown in Fig. 2, the degree of localization and isotropy vary from case to case.

The second important requirement is that the theory provide predictions for the positron annihilation characteristics. These include the positron lifetimes, momentum distributions of the annihilating electron-positron pairs, the energy levels of free and trapped positrons, and the trapping rates. The goal is that these characteristics be calculated from first principles without any adjustable parameters. This can be done, for example, on the basis of density-functional formalism. Therefore the results are real predictions independent of any empirical input and are therefore valuable in interpreting experimental results.

The aim of this review is to update the series of earlier general summaries of positron theory (Nieminen and Manninen, 1979; Nieminen, 1983), which are now about ten years old. There have been important developments since then in both bulk and surface applications of the positron techniques. We use a chronological order from the positron perspective and discuss first the models and results of detailed calculations for positron slowing down, diffusion, and trapping in Sec. II. The theoretical basis for the calculation of positron states in solids and on surfaces is presented in Sec. III. Finally, in Sec. IV, the results and ideas emerging from selected recent positron studies of condensed matter are reviewed.

\section{POSITRON-SOLID INTERACTION}

Positrons emanating from a radioactive source have a continuous energy spectrum characteristic of a beta decay, with end-point energies of the order of MeV's. There are now techniques available (see, e.g., Schultz and Lynn, 1988) where high-energy positrons from radioactive sources or pair production can be moderated to a monochromatic beam with controllable kinetic energies from a few $\mathrm{eV}$ up to the $\mathrm{keV}$ region. In either case, positrons rapidly lose their energy in condensed matter. At highest positron energies the most important process is ionization; i.e., the positron excites core electrons in collisions with the host atoms (Perkins and Carbotte, 1970). In the case of metals, the excitations of conduction electrons dominate at lower energies (Perkins and Carbotte, 1970). Above the plasmon threshold collective excitations can occur (Oliva, 1980). At lower energies the electron-hole excitations take over. Finally, when the positron energy has degraded to a fraction of an $\mathrm{eV}$, scattering off phonons dominates (Perkins and Carbotte, 1970). Eventually positrons reach thermal equilibrium with the host, with phonon emission and absorption maintaining the equilibrium. 
In the high-energy region the rate of energy transfer is very high. As a matter of fact, most of the time before thermalization is spent in the energy region just above thermal energies. Anyway, the thermalization process takes usually a rather short time compared to the positron lifetime in solids, which is of the order of a few hundred picoseconds. For example, it is estimated that a 1$\mathrm{keV}$ positron entering aluminum will thermalize within $\sim 3$ ps at $600 \mathrm{~K}$ and within $\sim 60$ ps at $10 \mathrm{~K}$ (Nieminen and Oliva, 1980). The rapidity of the thermalization is proven experimentally by angular correlation of annihilation radiation (ACAR) measurements (Kubica and Stewart, 1983; Hyodo et al., 1986), which show that the distribution of the annihilating positrons is thermal even at the temperature of $10 \mathrm{~K}$. Incomplete thermalization is, however, an important possibility if the positron can escape through a surface prior to equilibration. This is the case in low-energy positron beam experiments (Huomo et al., 1987).

When the positron has reached thermal equilibrium with the medium, its scattering is overwhelmingly dominated by phonons. This scattering is usually quasielastic and does not affect the average positron momentum distribution. Scattering by electrons is much less important, and impurity scattering starts to dominate only at very low temperatures (Bergersen et al., 1974). In a classical picture the movement of the positron is a nearly isotropic random walk (Nieminen and Oliva, 1980).

A practical way of describing the positron's slowing down and diffusing in solids is to regard the position $\mathbf{r}$ and the momentum $\mathbf{p}$ of the positron as stochastic variables and to calculate their probability density or distribution function $f(\mathbf{r}, \mathbf{p}, t)$ as a function of time $t$. The equation determining the distribution function is the famous Boltzmann equation, which can be written for positrons as

$$
\begin{aligned}
\frac{\partial f(\mathbf{r}, \mathbf{p}, t)}{\partial t} & +\mathbf{v}(\mathbf{p}) \cdot \nabla_{\mathbf{r}} f(\mathbf{r}, \mathbf{p}, t)+\mathbf{F} \cdot \nabla_{\mathbf{p}} f(\mathbf{r}, \mathbf{p}, t) \\
= & \left(\frac{\partial f(\mathbf{r}, \mathbf{p}, t)}{\partial t}\right]_{s}-\left(\lambda_{b}+\kappa\right) f(\mathbf{r}, \mathbf{p}, t)+f_{i}(\mathbf{r}, \mathbf{p}, t) .
\end{aligned}
$$

Above, $\mathbf{v}(\mathbf{p})$ is the positron velocity; $\mathbf{F}$, the force acting on the positron due to external fields; $\lambda_{b}$, the positron annihilation rate in the delocalized (bulk) state; $\kappa$, the positron trapping rate at defects; and $f_{i}(\mathbf{r}, \mathbf{p}, t)$, the source term. $[\partial f(\mathbf{r}, \mathbf{p}, t) / \partial t]_{s}$ denotes the total positronscattering rate and can be written as

$$
\begin{aligned}
{\left[\frac{\partial f(\mathbf{r}, \mathbf{p}, t)}{\partial t}\right]_{s}=\int d \mathbf{q}[} & R(\mathbf{q}, \mathbf{p}) f(\mathbf{r}, \mathbf{q}, t) \\
& -R(\mathbf{p}, \mathbf{q}) f(\mathbf{r}, \mathbf{p}, t)],
\end{aligned}
$$

where the first term in the integral describes the scattering from all states $q$ to the state $p$, and the second term describes the scattering from the state $\mathbf{p}$ to all other states q. Here, it is assumed that there is only one positron in the sample at a given time; so the Pauli exclusion principle need not be obeyed, and the distribution function $f(\mathbf{r}, \mathbf{p}, t)$ denotes merely the probability of finding the positron in the given state. The transition rate $R(\mathbf{p}, \mathbf{q})$ has to be determined for the different processes by calculating the relevant matrix elements of the interaction and taking the conservation laws into account. We illustrate this, in the following, with equations for the transition rates in a few important cases.

The transition rate for the conduction-electron excitations can be calculated using the positron-electron interaction screened in the random-phase approximation (RPA) in the low-momentum limit as (Woll and Carbotte, 1967; Jensen and Walker, 1990)

$R_{\mathrm{el}}(\mathbf{p}, \mathbf{q})=\frac{1}{\hbar \pi}\left(\frac{e^{2} a_{0}}{4 \pi k_{F}}\right)^{2} \int d \mathbf{k} \delta\left(\frac{\hbar^{2}(\mathbf{k}+\mathbf{p}-\mathbf{q})^{2}}{2 m_{e}}+\frac{\hbar^{2} \mathbf{q}^{2}}{2 m^{*}}-\frac{\hbar^{2} \mathbf{k}^{2}}{2 m_{e}}-\frac{\hbar^{2} \mathbf{p}^{2}}{2 m^{*}}\right]\left[1-f_{F}^{0}\left(\frac{\hbar^{2}(\mathbf{k}+\mathbf{p}-\mathbf{q})^{2}}{2 m_{e}}, T\right]\right] f_{F}^{0}\left[\frac{\hbar^{2} \mathbf{k}^{2}}{2 m_{e}}, T\right)$,

where $a_{0}$ is the Bohr radius; $k_{F}$, the Fermi wave vector; $m_{e}$, the free-electron mass; and $m^{*}$, the positron effective mass. $f_{F}^{0}(E, T)=\left\{\exp \left[\left(E-E_{F}\right) / k_{B} T\right]+1\right\}^{-1}$ is the equilibrium Fermi distribution with the Fermi energy $E_{F}$ and temperature $T$. The use of this approximation for the positron-electron scattering limits the applications to below the core ionization energies, i.e., to the energy region typically below $\sim 10 \mathrm{eV}$. The high-energy excitations are discussed in Sec. II.A.1.

Longitudinal-acoustic phonons usually dominate the scattering rate near thermal energies. In the Debye approximation (Perkins and Carbotte, 1970; Nieminen and Oliva, 1980; Jensen and Walker, 1990),

$$
\begin{aligned}
R_{\mathbf{p h}}(\mathbf{p}, \mathbf{q})= & \frac{\gamma^{2}}{4 \pi^{2}}|\mathbf{q}-\mathbf{p}|\left\{\left[f_{B}^{0}(\hbar s|\mathbf{q}-\mathbf{p}|, T)+1\right] \delta\left[\frac{\hbar^{2} \mathbf{q}^{2}}{2 m^{*}}-\frac{\hbar^{2} \mathbf{p}^{2}}{2 m^{*}}-\hbar s|\mathbf{q}-\mathbf{p}|\right]\right. \\
& \left.+f_{B}^{0}(\hbar s|\mathbf{q}-\mathbf{p}|, T) \delta\left[\frac{\hbar^{2} \mathbf{q}^{2}}{2 m^{*}}-\frac{\hbar^{2} \mathbf{p}^{2}}{2 m^{*}}-\hbar s|\mathbf{q}+\mathbf{p}|\right]\right\}\left(\omega_{D}-s|\mathbf{q}-\mathbf{p}|\right)
\end{aligned}
$$


where $s$ is the sound velocity, $\omega_{D}$ is the Debye frequency, $f_{B}^{0}(E, T)=\left[\exp \left(E / k_{B} T\right)-1\right]^{-1}$ is the equilibrium Bose distribution, and $\Theta$ is the step function. The positronphonon coupling constant $\gamma$ can be calculated for the longitudinal-acoustic phonons in the deformationpotential approximation as

$$
\gamma=\frac{E_{d}}{(2 \rho s)^{1 / 2}}=\frac{E_{d} s^{1 / 2}}{\left(2 \Omega\left\langle c_{i i}\right\rangle\right)^{1 / 2}},
$$

where $\rho$ is the mass density and $\left\langle c_{i i}\right\rangle$ is the elastic constant associated with longitudinal waves and averaged over the directions of sound propagation. $E_{d}$ is the de- formation potential defined as

$$
E_{d}=V \frac{\partial E_{+}}{\partial V}
$$

where $E_{+}$is the total energy of the crystal with the positron in its lowest state (Bloch state at $\mathbf{k}=0$ ), and $V$ is the crystal volume. In practice the deformation potential can be calculated from the volume derivatives of the electron and positron chemical potentials (Sec. IV.A).

The scattering rate due to neutral substitutional impurities can be calculated from the matrix element of the potential difference $V_{I}(\mathbf{r})-V_{H}(\mathbf{r})$ between the impurity and the host atoms (Bergersen et al., 1974),

$$
R_{\text {imp }}(\mathbf{p}, \mathbf{q})=\frac{2 \pi}{\hbar}\left|\int d \mathbf{r} \Psi_{\mathbf{q}}^{+*}(\mathbf{r})\left[V_{I}(\mathbf{r})-V_{H}(\mathbf{r})\right] \psi_{\mathbf{p}}^{+}(\mathbf{r})\right|^{2} \delta\left[\frac{\hbar^{2} \mathbf{q}^{2}}{2 m^{*}}-\frac{\hbar^{2} \mathbf{p}^{2}}{2 m^{*}}\right]
$$

Above, $\psi_{\mathrm{p}}^{+}(\mathbf{r})$ is the positron Bloch-state wave function, and $\Psi_{q}^{+}(\mathbf{r})$ is the positron wave function in the presence of the impurity.

In semiconductors, the scattering of electrons and holes by charged impurities is an important effect at low temperatures. The scattering of positrons by ionized impurities has been studied in the case of phosphorus-doped silicon (Soininen et al., 1992). In this scattering process, a charged impurity causes a long-range Coulombic perturbation. This leads to long-range distortion of positron wave function, and the low-energy scattering cross section can become very large. In reality, there are effects, such as screening or uncertainty broadening, that lower the scattering cross section for charged impurities (see, for example, Ridley, 1988).

In semiconductors and insulators, electron-hole excitations with an energy less than the energy of the band gap are impossible. However, in the case of semiconductors with a narrow gap, this does not hinder thermalization appreciably; i.e., the thermalization time is of the same order as in metals (Jorch et al., 1984). In the case of wide-band-gap insulators, electron-hole excitations are no longer possible when the positron energy reaches the region of a few eV's. One-phonon processes degrade the energy inefficiently, and the probability of multiphonon scattering is very small. Positrons in insulators may therefore not have enough time to reach thermal equilibrium before annihilation, trapping into defects, or reemission into vacuum (Mills and Crane, 1985; Gullikson and Mills, 1986; Lynn and Nielsen, 1987). In a wide-gap insulator a positron with kinetic energy less than the band gap can also lose energy in positronium (Ps) formation. In this process, it is energetically possible to excite an electron from the valence band because, in the final state, the Ps binding energy (6.8 eV in vacuum) is gained. Thus the positron energy region where Ps formation is most likely to occur is the so-called Ore gap (Ore, 1949),

$$
E_{g}-E_{P s}<E<E_{g}
$$

where $E_{g}$ and $E_{\mathrm{Ps}}$ are the band gap and the Ps binding energy, respectively.

The various scattering channels, including those mentioned above, are discussed in more detail below. We shall describe the different stages of the positron-solid interaction and the methods for modeling them quantitatively. The thermalization and diffusion stages are separated and, finally, the trapping into defects is dealt with.

\section{A. Before thermal equilibrium}

The penetration of high-energy positrons emitted from a radioactive source into a solid can be described by an empirical law first established for electrons (Gleason et al., 1951) and later shown to be valid also for positrons (Brandt and Paulin, 1977; Paulin, 1979). It states that the positron intensity $I(z)$ decays exponentially with the depth $z$ as

$$
I(z)=I_{0} \exp \left(-\alpha_{+} z\right), \quad \alpha_{+} \approx 17 \frac{\rho\left(\mathrm{g} / \mathrm{cm}^{3}\right)}{E_{\max }^{1.43}(\mathrm{MeV})}\left(\mathrm{cm}^{-1}\right),
$$

where $E_{\max }$ is the maximum energy of the emitted positrons. Thus positrons from a nuclear $\beta$ emitter annihilate in the solid within a depth of the order of a millimeter, and therefore they probe bulk properties.

The above approximation for the penetration depth is an important planning parameter for the samples in positron lifetime spectroscopy. In investigations of nearsurface properties of solids using monoenergetic positron beams, a more quantitative description of positron penetration is needed for the starting point of the data analysis - namely, the positron implantation profile; i.e., the positron spatial distribution after thermalization but before the diffusion stage is needed for different materials and for different incident positron energies. These im- 
plantation profiles have been studied both theoretically and experimentally.

\section{High-energy region}

In the Monte Carlo (MC) methods the slowing-down histories of a large number of individual positrons are simulated. The initial conditions and the scattering events for each positron are generated from the relevant cross sections. Collecting the results of all the simulated positron histories generates the distribution functions desired. The individual positrons are usually followed down to energies of about $10-20 \mathrm{eV}$, where the core ionization processes stop. The resulting real-space distribution is then the positron implantation profile. Recently, the inelastic-scattering processes with valence electrons have also been taken into account (Coleman et al., 1992; Massoumi et al., 1992, 1993). This is done using dielectric formalism, the benefit of which is that it accounts for scattering off both core and valence electrons, including electron-hole-pair and plasmon excitations.

Valkealahti and Nieminen $(1983,1984)$ have made a comprehensive set of MC simulations for positrons. They (1984) treated the elastic scattering from the atoms by calculating exact cross sections for effective potentials of the atom in the crystalline environment. They treated the ionization part of inelastic scattering using Gryziński's (1965) semiempirical expression, in which each bound-electron level is treated separately. Furthermore, Valkealahti, and Nieminen (1984) approximated those excitation processes that do not lead to ionization. The threshold energy for excitation was used as a parameter, which was fitted to give the Bethe formula (Bethe
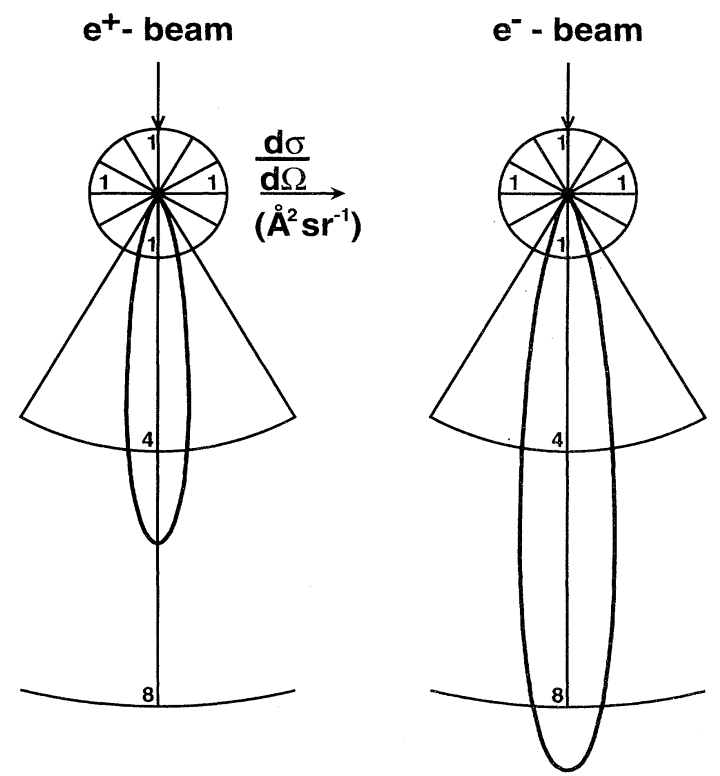

FIG. 3. Polar plots of the differential elastic-scattering cross section of $1-\mathrm{keV}$ positrons and electrons off a copper atom in a crystalline environment (from Valkealahti and Nieminen, 1984). and Ashkin, 1953) for the stopping cross section at high energies. The computer code by Valkealahti and Nieminen is available in a software server (SOFTWARE, 1993).

Figure 3 shows the differential elastic-scattering cross sections obtained by Valkealahti and Nieminen (1984) for positrons and electrons in $\mathrm{Cu}$ when the kinetic energy of the particle is $1 \mathrm{keV}$. The important difference between positrons and electrons is that the cross section for electrons is much larger than that for positrons. Moreover, especially at low particle energies, electrons have a higher probability of scattering to large angles $\left(>30^{\circ}\right)$ than have positrons. These differences are due to the fact that the electron feels an attractive interaction with the atom, whereas the interaction between the positron and the atom is repulsive. The interaction for electrons also contains an exchange part, whereas for positrons it does not.

The MC simulations (Valkealahti and Nieminen, 1984) predict quantities that can be directly compared with experiments. The agreement between simulations and experiments is usually very good. For example, the simulated and measured transmission probabilities for positrons and electrons through thin aluminum films at different incident energies are shown in Fig. 4 as a function of film thickness. Figure 5 gives the backscattering probabilities from a semi-infinite aluminum as a function of particle energy. The transmission probabilities for positrons are higher than those for electrons. Correspondingly, backscattering for positrons is lower than that for electrons. These differences originate from the larger differential elastic-scattering cross section for electrons and from the fact that electrons have a higher probability of scattering to large angles.

The positron backscattering probabilities from solid surfaces have been measured and simulated recently by

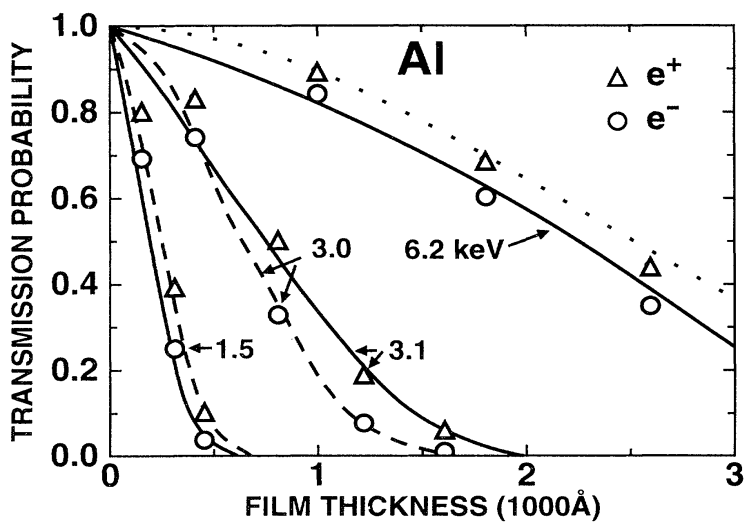

FIG. 4. Transmission probabilities for positrons and electrons with different kinetic energies through thin aluminum films as a function of film thickness. The circles and triangles are simulated results for electrons and positrons, respectively. The solid and dashed lines are the experimental probabilities for positrons and electrons, respectively (from Valkealahti and Nieminen, 1984). 


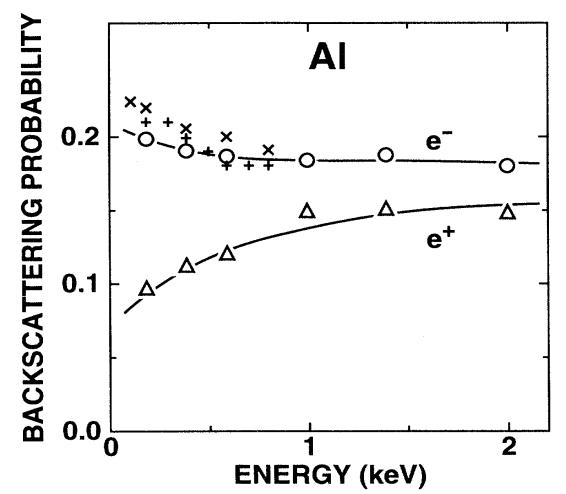

FIG. 5. Backscattering probabilities for positrons and electrons from semi-infinite aluminum as a function of the projectile energy. The crosses and pluses are experimental results for electrons, whereas the circles and triangles are simulated results for electrons and positrons, respectively. The lines are guides for the eye (from Valkealahti and Nieminen, 1984).

several groups (Massoumi et al., 1991, 1992, 1993; Coleman et al., 1992; Mäkinen, Palko, et al., 1992). Massoumi et al. (1991, 1992, 1993) have measured the energy and angle-resolved probabilities for several elemental solids. Massoumi et al. $(1992,1993)$ and Coleman et al. (1992) have performed Monte Carlo simulations that differ from those by Valkealahti and Nieminen (1984) in the treatment of the inelastic electron scattering. In their simulations the inelastic electron-scattering probability is proportional to $\operatorname{Im}[1 / \epsilon(\mathbf{q}, \omega)]$, where $\hbar \mathbf{q}$ and $\hbar \omega$ are the momentum and energy transfers, respectively. $\epsilon(\mathbf{q}, \omega)$ is the dielectric function for which the form by Penn (1987) is used. Penn's dielectric function is a weighted average of Lindhard functions for different electron-gas densities. The weights are derived from experimental optical data, which include excitations from low energies of the order of $0.1 \mathrm{eV}$ up to high energies of the order of several $\mathrm{keV}$ 's. Thus the formalism includes both the core and valence-electron processes and both the one-particle excitations and collective plasmon modes. The method is described in detail in the recent article by Jensen and Walker (1993).

Mäkinen, Palko, et al. (1992) compared the measured backscattering probabilities with Monte Carlo results obtained using the original approach by Valkealahti and Nieminen (1984). Figure 6 shows their results for the energy dependence for $\mathrm{C}, \mathrm{Si}, \mathrm{Ge}$, and $\mathrm{Au}$. The agreement between experiment and theory is fairly good for both the light Si and the heavy Au targets.

Among the useful results from the MC simulations are the positron implantation profiles. Valkealahti and Nieminen (1984) determined them for various solid hosts and incident positron energies by simulating the slowing of about 2000 positrons down to the energy of $20 \mathrm{eV}$ and then recording their trajectory end points. The profiles are not very sensitive to moderate changes in the final energy (e.g., the energy of $100 \mathrm{eV}$ gives essentially the same

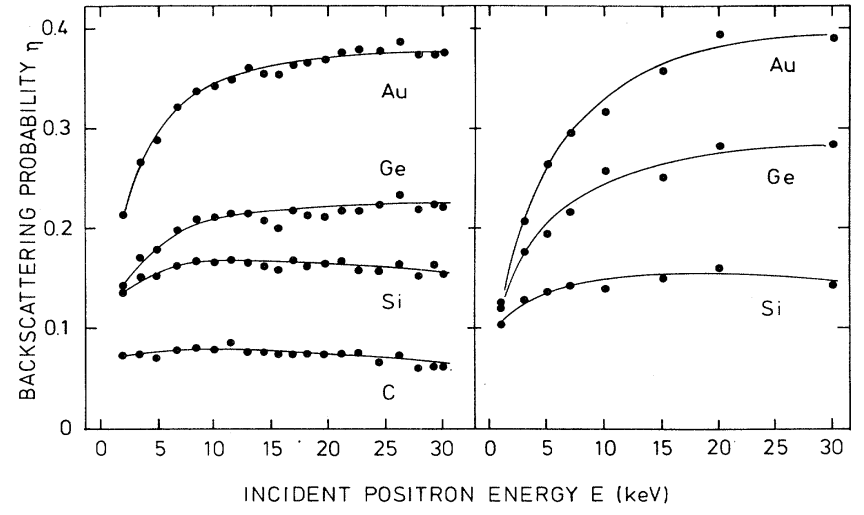

FIG. 6. Backscattering probabilities for positrons from graphite, $\mathrm{Si}, \mathrm{Ge}$, and $\mathrm{Au}$ : left, measured results; right, Monte Carlo simulations (from Mäkinen, Palko, et al., 1992).

results as $20 \mathrm{eV}$; Valkealahti and Nieminen, 1984). As an example of the distribution of the trajectory end points, Fig. 7 shows the case of $5-\mathrm{keV}$ positrons hitting perpendicularly an aluminum surface. The distribution has cylindrical symmetry and resembles deeper in bulk a hemisphere. Figure 8 shows the corresponding stopping or implantation profiles as a function of the distance from the surface. In the figure the simulated curves are also compared with experiments (Mills and Wilson, 1982). The experimental values are above the simulated ones near the surface, and because the areas below the curves are the same by normalization, the maxima of the simulated profiles are higher than the experimental ones. The discrepancy arises because the experimental results are deduced by differentiating the transmission probabilities through thin films, whereas the simulation uses the correct semi-finite geometry. The experimental results are affected by the increase of the backscattering with increasing film thickness and by the fact that the positrons that have penetrated through the film cannot return to it,

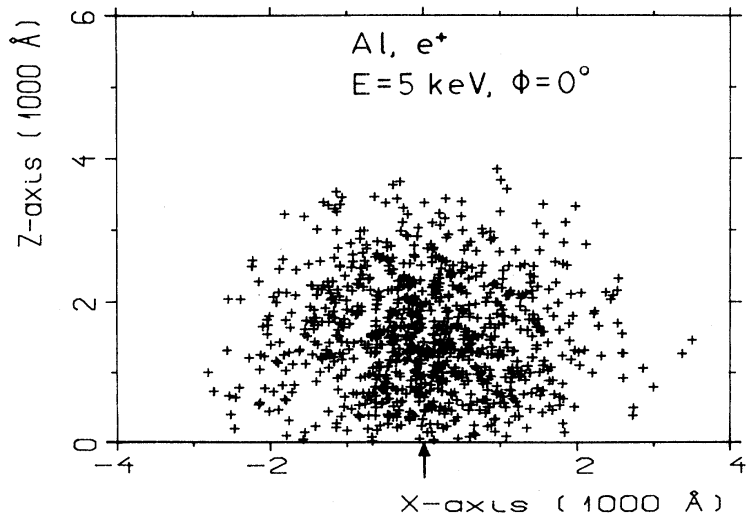

FIG. 7. Distribution of simulated trajectory end points for 5$\mathrm{keV}$ positrons hitting the Al surface perpendicularly. The arrow indicates the entrance point of positrons (from Valkealahti and Nieminen, 1984). 


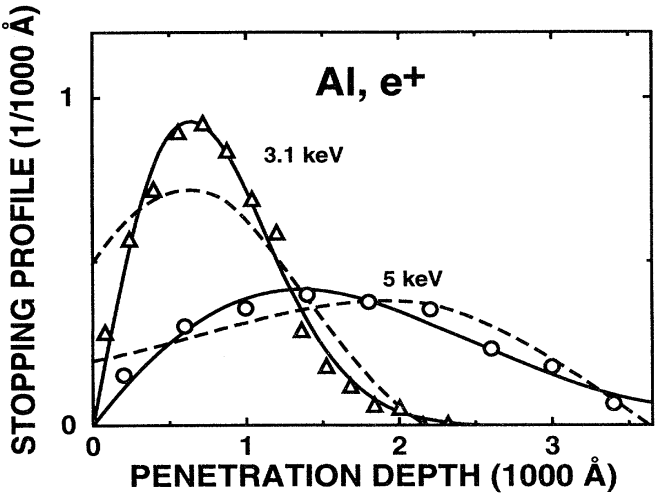

FIG. 8. Simulated stopping profiles for 3.1- (triangles) and 5$\mathrm{keV}$ (circles) positrons in semi-infinite aluminum. These profiles were obtained by reducing two-dimensional data similar to Fig. 7 to one dimension. The solid lines are the corresponding fits to the Makhovian function [Eq. (11)]. The dashed lines were obtained as derivatives of the experimental transmission probabilities for thin aluminum films. The area below each curve is the same constant by normalization (from Valkealahti and Nieminen, 1984).

whereas in the semi-infinite geometry positrons can always return to a given depth, which they have passed.

The simulated implantation profiles (Valkealahti and Nieminen, 1984) obey well the form originally suggested by Makhov (1961) for electrons. The transmission probability $\eta_{T}$ for the projectile particles as a function of the distance $z$ can be approximated as

$$
\eta_{T}(z) \simeq \exp \left[-\left(\frac{z}{z_{0}}\right)^{m}\right]
$$

and the implantation profile is then obtained by derivation as

$$
P(z)=\frac{m z^{m-1}}{z_{0}^{m}} \exp \left[-\left(\frac{z}{z_{0}}\right)^{m}\right] .
$$

Here $m$ is a dimensionless parameter and $z_{0}$ is related to the mean stopping depth $\bar{z}$ by

$$
z_{0}=\frac{\bar{z}}{\Gamma[(1 / m)+1]},
$$

where $\Gamma(x)$ is the gamma function. If one sets $m=2$, a derivative of a Gaussian is obtained, while $m=1$ gives an exponential profile.

The solid curves shown in Fig. 8 are just fits with the Makhov function (11). In the case of metals the fitted $m$ values are slightly below 2 . Moreover, the mean penetration depth depends on the incident positron energy rather accurately as the power law (suggested by Mills and Wilson, 1982)

$$
\bar{z}=A E^{n} \text {. }
$$

According to simulations, the $n$ values lie around 1.6 , but can depend somewhat on the material and the energy range.

A collection of the Makhovian fit parameters $A, n$, and $m$ accrued during the last decade is demonstrated in Table I. First, the values obtained by Valkealahti and Nieminen (1984) for gaseous $N_{2}$ and metals $\mathrm{Al}, \mathrm{Cu}$, and $\mathrm{Au}$ are given. The results by Soininen et al. (1992) for $\mathrm{Al}, \mathrm{Si}, \mathrm{Ge}$, and Au obtained using similar simulations are also shown in Table I. Soininen et al. (1992) found that the values of these parameters are independent of the incident energy in the range $3-30 \mathrm{keV}$. Finally, the parameters of the Makhovian fits calculated by Jensen and Walker (1993) from the data obtained by using the Penn dielectric loss function are shown for $\mathrm{Al}, \mathrm{Cu}, \mathrm{Ag}$, and Au. The parameters $A, n$, and $m$ determine the implantation profiles uniquely.

Mills and Wilson (1982) tried to estimate the stopping profiles directly with thin metal foils. They determined the mean penetration depths and obtained, corresponding to the positron energies between 1 and $6 \mathrm{keV}$, the values of $1.60_{-0.08}^{+0.15}$ and $1.43_{-0.11}^{+0.07}$ for $n$ in the case of Al and $\mathrm{Cu}$, respectively. Vehanen et al. (1987) measured the annihilation line-shape parameter in multilayer structures like $\mathrm{Al}_{2} \mathrm{O}_{3} / \mathrm{ZnS} / \mathrm{Al}_{2} \mathrm{O}_{3}$ as a function of positron implantation energy. The annihilation shape parameter has a different value for each different substrate layer, and it turned out that the measured effective parameter is very sensitive to the form of the implantation profile. Vehanen et al. (1987) concluded that the implantation profile is close to the derivative of a Gaussian $(m=2.0 \pm 0.1)$. The other parameters from their analysis, i.e., $A=4.0 \pm 0.3 \mu \mathrm{g} / \mathrm{cm}^{2}$ and $n=1.62 \pm 0.05$,

TABLE I. Parameters of the Makhovian function [Eq. (11)] fits to simulated implantation profiles.

\begin{tabular}{ccccc}
\hline \hline Material & $A\left(\mu \mathrm{g} / \mathrm{cm}^{2} \mathrm{keV}^{-n}\right)$ & $n$ & $m$ & Footnote \\
\hline $\mathrm{N}_{2}$ & 3.3 & 1.71 & 2.05 & $\mathrm{a}$ \\
$\mathrm{Al}$ & 3.7 & 1.67 & 1.92 & $\mathrm{a}$ \\
$\mathrm{Cu}$ & 5.0 & 1.54 & 1.83 & $\mathrm{a}$ \\
$\mathrm{Au}$ & 10.6 & 1.32 & 1.72 & $\mathrm{a}$ \\
$\mathrm{Al}$ & & & & \\
$\mathrm{Si}$ & 3.4 & 1.69 & 1.94 & $\mathrm{~b}$ \\
$\mathrm{Ge}$ & 3.3 & 1.69 & 1.91 & $\mathrm{~b}$ \\
$\mathrm{Au}$ & 4.3 & 1.60 & 1.78 & $\mathrm{~b}$ \\
& 7.4 & 1.48 & 1.70 & $\mathrm{~b}$ \\
$\mathrm{Al}$ & & & & \\
$\mathrm{Cu}$ & 2.64 & 1.74 & 1.97 & $\mathrm{c}$ \\
$\mathrm{Ag}$ & 3.78 & 1.61 & 1.78 & $\mathrm{c}$ \\
$\mathrm{Au}$ & 3.98 & 1.57 & 1.76 & $\mathrm{c}$ \\
& 6.58 & 1.49 & 1.71 & $\mathrm{c}$ \\
$\mathrm{Al}$ & & & & \\
$\mathrm{Cu}$ & 3.3 & 1.55 & & $\mathrm{~d}$ \\
$\mathrm{Au}$ & 5.6 & 1.52 & & $\mathrm{~d}$ \\
\hline \hline
\end{tabular}

${ }^{a}$ Valkealahti and Nieminen, 1984.

boininen et al., 1992.

'Jensen and Walker, 1993.

${ }^{\mathrm{d}}$ Ritley et al., 1993. 
are in fair agreement with the simulated ones given in Table I.

Baker et al. (1991a) determined the implantation profiles experimentally and by Monte Carlo simulations. They measured the Doppler broadening line shape for the annihilation radiation as a function of implantation energy and the thickness of an Al overlayer on a glass substrate. From the diffusion-corrected data, the implantation profile was directly obtained. Their Monte Carlo simulation differed from the Valkealahti-Nieminen work $(1983,1984)$ in that it utilized the conduction-electron cross section calculated in the dielectric random-phase approximation. Thus the simulation is based on theoretical first-principles data without any adjustable parameters. The simulated profiles are in good agreement with the measured ones, but Baker et al. found that the Makhov profiles do not accurately describe all the details of the profiles. Baker et al. (1991a) as well as Jensen and Walker (1993) and Ritley et al. (1993) later, suggested modifying the Makhovian fit. The adapted form is

$$
P(z)=-\frac{d}{d z} \exp \left\{-\left[\frac{z}{z_{0}^{*}}\left[1+\frac{z}{z_{0}^{*}}\right]^{2}\right]^{m^{*}}\right\}
$$

One of the benefits of this form is that it can describe finite positron densities at the surface (Ritley et al., 1993). Jensen and Walker (1993) found that for a given material the fitting parameters $m^{*}$ and $z_{0}^{*} / \bar{z}$ depend only slightly on the energy within the range $5-30 \mathrm{keV}$. Moreover, they found that the modified Makhovian provides a better fit for the light materials like Al than the original one, whereas for the heavier materials like noble metals, the opposite is true.

Baker et al. later made more measurements for $\mathrm{Al}$ (Baker, Chilton, and Coleman, 1991) and for $\mathrm{Au}$, with Monte Carlo simulations using the Penn model (Baker et al., 1991b). They concluded that the Makhov function gives reasonable fits provided that the power law (13) for the penetration depth is replaced by an energy dependence fitted to experiments or simulations. They found that $m=2$ gives a reasonable fit for $\mathrm{Al}$, whereas $\mathrm{Au}$ requires $m=1.7$. These numbers should be compared to those in Table I.

\section{Low-energy region}

Jensen and Walker (1990) have studied the late stages of the slowing down of positrons by applying the Boltzmann equation. They included electron scattering within the low-energy and low-momentum limit of the random-phase approximation [RPA; Eq. (3)], which limits the application to the energy region below $\sim 10 \mathrm{eV}$. They included acoustic-phonon scattering in the Debye model [Eq. (4)]. The model parameters were chosen to correspond to aluminum. The approximations made in the electron scattering were argued not to be severe, because most of the time during thermalization is spent at low energies, where phonon scattering dominates. Jensen and Walker applied the Boltzmann equation for a homogeneous medium; i.e., their positron distribution function depended on the positron momentum and time, only. Thus the second term in the Boltzmann equation (1) vanishes and, because they did not consider the effects of external fields, the third term in Eq. (1) equals zero.

Figure 9 shows the mean positron energy $\bar{E}$ as a function of time, calculated by Jensen and Walker (1990). The initial positron momentum distribution $\left[f_{i}(\mathbf{r}, \mathbf{p}, t)\right.$ in Eq. (1)] is a narrow Gaussian corresponding to a mean energy of $10 \mathrm{eV}$, and the time dependence has been replaced by a delta function. At first the slowing down is similar at each temperature shown, down to the mean energy of $\sim 0.3 \mathrm{eV}$. Thereafter the curves approach asymptotically the thermal energies $\frac{3}{2} k_{B} T$. It is interesting that the early results by Lee-Whiting (1955) are in good agreement with those shown in Fig. 9. Lee-Whiting (1955) calculated the time-dependent positron energy by considering only the electron-hole excitation processes. The energy-loss rate $(d \bar{E} / d t)(E=\bar{E})$ corresponding to the 300-K curve in Fig. 9 is shown in Fig. 10. This rate is compared with the energy-loss rate $(d E / d t)$ $\left(E=p^{2} / 2 m_{e}\right)$ obtained when the positron has a fixed momentum $p$. The rate $(d E / d t)\left(E=p^{2} / 2 m_{e}\right)$ assumes that the positron momentum distribution is a delta function at each time during the thermalization process, whereas in the case of $(d \bar{E} / d t)(E=\bar{E})$ the positron momentum distribution is calculated from the Boltzmann equation. Jensen and Walker pointed out that the latter is the correct physical quantity to be used in accurate calculations of positron thermalization effects. Note that rate $d \bar{E} / d t$ goes to zero at the correct thermal energy $\left(\frac{3}{2} k_{B} T\right)$, whereas $d E / d t$ vanishes at the higher energy of $2 k_{B} T$.

Steady-state momentum distributions from which positrons annihilate are shown in Fig. 11. Distributions obtained by Jensen and Walker (1990), with the abovementioned approximations, and by Hyodo et al. (1984) for the temperatures of 300 and $10 \mathrm{~K}$ are shown. The

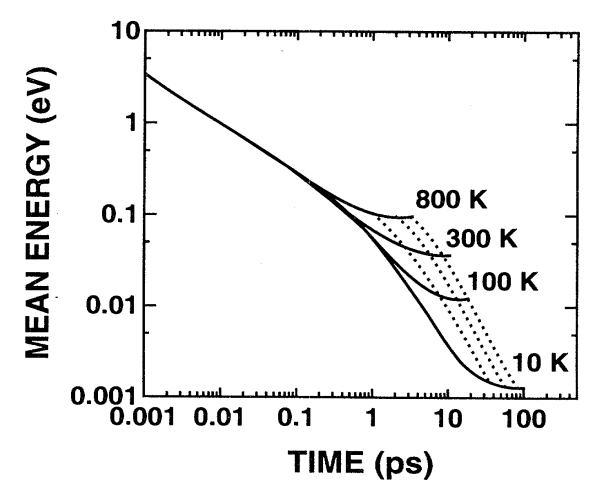

FIG. 9. Positron mean energy $\bar{E}$ as a function of time after implantation in $\mathrm{Al}$ at different temperatures. The dotted lines connect points corresponding to $\bar{E}=1.1 E_{\text {th }}, \bar{E}=1.01 E_{\text {th }}$, and $\bar{E}=1.001 E_{\text {th }}$, where $E_{\text {th }}$ is the thermal energy $\frac{3}{2} k_{B} T$ (from Jensen and Walker, 1990). 


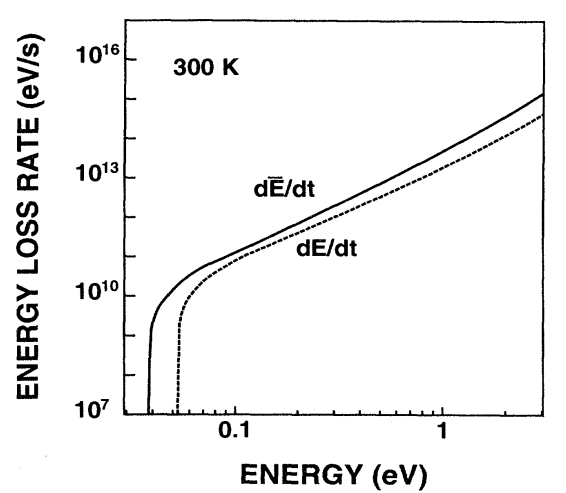

FIG. 10. Time derivative of the positron mean energy $d \bar{E} / d t$ as a function of the mean energy $\bar{E}$, and the average energy-loss rate $d E / d t$ for positrons with energy $E=p^{2} / 2 m_{e}$. The temperature is $300 \mathrm{~K}$ (from Jensen and Walker, 1990).

distributions consist of a Maxwell-Boltzmann-like part and a low intensity tail. In the calculation by Jensen and Walker (1990), the tail is due to the positrons that annihilate before thermalization, whereas in Hyodo et al. a $p^{-2}$ tail is due to the dressing of positrons by phonons, with nonthermal effects neglected altogether. It is seen that the nonthermal effects are more important at low temperatures, whereas at high temperatures the $p^{-2}$ tail dominates.

Recently, Ritley et al. (1993) made Monte Carlo simulations for the low-positron-energy region. They started from the stopping profiles obtained by using the positron end-point energy of $25 \mathrm{eV}$ (Asoka-Kumar and Lynn, 1990; Ritley et al., 1990; Ghosh et al., 1992, 1993). In the simulations, they took into account the inelastic scattering with valence electrons and with longitudinalacoustic phonons. The former were modeled by the Penn mean free path and RPA approach and the latter with the deformation-potential approach. Moreover, Ritley et al. (1993) included the energy step at the surface (the positron work function). As a matter of fact, these Monte Carlo simulations represent a direct and efficient way to integrate the Boltzmann equation discussed in the context of the work by Jensen and Walker (1990). Ritley et al. (1993) were able to distinguish the transition from the thermalization regime to the diffusion regime, and the diffusion coefficients determined are in reasonable agreement with experiments. They show that for low initial positron energies (less than approximately $10 \mathrm{keV}$ ), the positron distributions broaden remarkably before thermalization when the processes below $25 \mathrm{eV}$ are taken into account. The positive work function leads in this energy region to a nonvanishing positron density at the surface, when the thermalized positrons have no energy to escape into the vacuum. Ritley et al. showed that the modified Makhovian function [Eq. (14)] gives a good description of the positron distribution after full thermalization. Moreover, they showed that the power law of Eq. (13) is not valid for low incident positron energies, but energy dependence should be used for the $A$ parameter.

Huttunen et al. (1989) have extracted the positron mean free paths for elastic- and inelastic-scattering processes by analyzing the experimental data of Gidley and Frieze (1988). In the experiment, thermalized positrons from bulk $\mathrm{Ni}$ enter a $\mathrm{Cu}$ overlayer. Because of the difference in positron energy levels between the substrate and the overlayer (positron affinity difference, Sec. IV.A.1), the positrons entering the overlayer have a kinetic energy much larger than the thermal energy: they are "hot" positrons in the overlayer. These hot positrons can suffer both inelastic- and elastic-scattering processes before emission into vacuum. In the experiment (Gidley and Frieze, 1988), the fraction of the positrons transmitted elastically through the overlayer is monitored as a function of the overlayer thickness. Huttunen et al. (1989) made a two-flux approximation (positrons moving inwards or outwards in the overlayer) for the Boltzmann equation and obtained for the fraction of the elastically transmitted positrons a form, in which the most important parameters are the inelastic and elastic mean free paths. Fitting this form to the experimental data, Huttunen et al. (1989) obtained for the hot positrons with $0.5 \mathrm{eV}$ the values of $300 \pm 100 \AA$ and $20 \pm 5 \AA$ for the inelastic and elastic mean free paths, respectively. These values are in good agreement with the theoretical predictions (Nieminen and Oliva, 1980) of $350 \AA$ and 35 $\AA$ for the electron-hole-pair excitations and acousticphonon scattering, respectively.
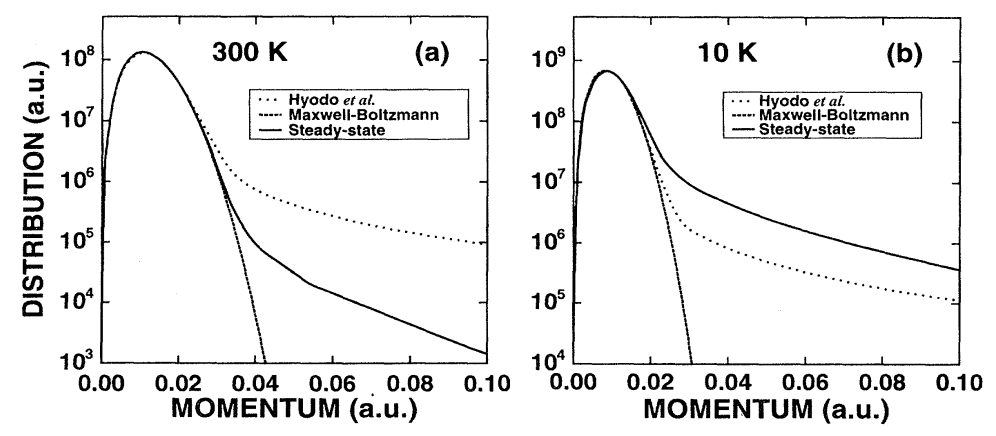

FIG. 11. Steady-state positron momentum distributions at $300 \mathrm{~K}$ (a) and $10 \mathrm{~K}$ (b). Results by Jensen and Walker (1990; solid line) and Hyodo et al. (1986; dotted line) are shown. The thermal Maxwell-Boltzmann distributions (dashed line) are shown for comparison. The curves in each panel are normalized to have the same total area (from Jensen and Walker, 1990). 


\section{B. During thermal equilibrium}

\section{Positron diffusion equation}

For positrons in thermal equilibrium with the host, the momentum distribution has reached the timeindependent form. The evolution of the positron spatial distribution is then conventionally described by the diffusion-annihilation equation

$$
\begin{aligned}
\frac{\partial f(\mathbf{r}, t)}{\partial t}= & D_{+} \nabla^{2} f(\mathbf{r}, t)-\left[\lambda_{b}+\kappa(\mathbf{r})\right] f(\mathbf{r}, t) \\
& -\nabla \cdot\left[\mathbf{v}_{d}(\mathbf{r}) f(\mathbf{r}, t)\right]+f_{i}(\mathbf{r}, t),
\end{aligned}
$$

where $D_{+}$is the diffusion coefficient and $\mathbf{v}_{d}$ is the positron drift velocity due to external fields. The trapping rate is now generalized to have a possible spatial dependence. The diffusion equation can be obtained from the Boltzmann equation (1) by integrating over the positron momentum and replacing the microscopic positron current density $\mathbf{j}$ with the expression for Fick's law, i.e.,

$$
\begin{aligned}
\nabla_{\mathbf{r}} \cdot \int d \mathbf{p} \frac{\mathbf{p}}{m^{*}} & f(\mathbf{r}, \mathbf{p}, t) \\
& =\nabla_{\mathbf{r}} \cdot \mathbf{j} \\
& =\nabla_{\mathbf{r}} \cdot\left[-D_{+} \nabla_{\mathbf{r}} f(\mathbf{r}, t)+\mathbf{v}_{d}(\mathbf{r}) f(\mathbf{r}, t)\right] .
\end{aligned}
$$

The diffusion coefficient can, in principle, be calculated from the microscopic quantum-mechanical theory when the scattering processes are known (Forster, 1975). The semiclassical random-walk theory gives, however, a more transparent equation,

$$
D_{+}=\frac{\left\langle v^{2}\right\rangle^{1 / 2} l}{3}=\frac{\left\langle v^{2}\right\rangle \tau}{3}
$$

where $\left\langle v^{2}\right\rangle$ is the average of the square of the positron thermal velocity $\left(\left\langle v^{2}\right\rangle=3 k_{B} T / m^{*}\right), l$ is the positron mean free path between scattering events, and $\tau$ is the corresponding relaxation time. The relaxation time should now be determined from the microscopic scattering rates, e.g., from Eqs. (3)-(5). Equation (17) thus assumes that the relaxation-time approximation in the Boltzmann equation is valid. This is true for elastic scattering, i.e., scattering off impurities; for quasielastic scattering off acoustic phonons; and for velocityrandomizing scattering off optical phonons and electrons (McMullen, 1985). Furthermore, the diffusion equation is usually solved at the time-independent steady-state limit, which reads

$$
\begin{aligned}
& D_{+} \nabla^{2} f(\mathbf{r})-\left[\lambda_{b}+\kappa(\mathbf{r})\right] f(\mathbf{r}) \\
&-\nabla \cdot\left[\mathbf{v}_{d}(\mathbf{r}) f(\mathbf{r})\right]+f_{i}(\mathbf{r})=0
\end{aligned}
$$

The time-independent diffusion equation (18) can be obtained in an alternative picture, which follows the fate of a single positron. At $t=0$ a single positron is introduced into the system and the positron spatial probability distribution is the implantation profile $f_{i}(\mathbf{r})$. The devel- opment of the distribution $f(r, t)$ obeys the diffusionannihilation equation (15) with source term $f_{i}(\mathbf{r}, t)=0$. Equation (18) is then obtained by integrating over time using the initial condition

$$
f(\mathbf{r}, t=0)=f_{i}(\mathbf{r})
$$

and denoting (the steady-state positron distribution)

$$
\int_{0}^{\infty} d t f(\mathbf{r}, t)=f(\mathbf{r}) .
$$

The sum of the positron annihilation rate $\lambda_{b}$ and the trapping rate $\kappa$ define the effective positron lifetime $\tau_{\text {eff }}(\mathbf{r})$ in the delocalized state. Thus

$$
1 / \tau_{\text {eff }}(\mathbf{r}) \equiv \lambda_{\text {eff }}(\mathbf{r})=\lambda_{b}+\kappa(\mathbf{r}) .
$$

The positron bulk lifetime $\tau_{b}=1 / \lambda_{b}$ refers to the defectfree lattice and is constant for the given material. The trapping rate $\kappa(\mathbf{r})$ depends on the types of defects in the sample and is proportional to the defect concentration. The defect concentration may vary over the sample.

The diffusion picture is classical and applies only if certain conditions have been fulfilled (Brandt and Arista, 1979). Firstly, it is assumed that the positron momentum distribution has to obey Maxwell-Boltzmann statistics. Secondly, the scattering of positrons has to be quasielastic and isotropic. Thirdly, the length scales, e.g., the thickness of a material layer or a defect zone (Huttunen et al., 1989), or the penetration depth for monoenergetic positrons, have to be large enough. The validity of the random-walk theory (relaxation-time approximation) requires that the sample length scales be longer than the mean free path for scattering. If these conditions are not fulfilled, one has to fall back to the solution of the Boltzmann equation, in the most stringent case without making the relaxation-time approximation by, for example, MC methods. At room temperature the positron mean free path in metals is typically of the order of $10^{2}$ $\AA$, whereas the total diffusion length before annihilation, $L_{+}=\sqrt{D_{+} /\left(\lambda_{b}+\kappa\right)}$, is of the order of $10^{3} \AA$. The positron wave character defines another length scale for localization. The positron thermal wavelength is

$$
\Lambda_{+}=h / \sqrt{3 m^{*} k_{B} T} \approx 50 \sqrt{300 \mathrm{~K} / T} \AA .
$$

In describing the slow-positron-beam experiments, the diffusion equation simplifies further. If the material properties, such as the defect density, depend only on the distance from the surface, one obtains a one-dimensional equation with the distance $z$ from the entrance surface as the variable. Moreover, in metals the macroscopic electric fields vanish due to a perfect screening, and therefore the drift term in Eqs. (15) and (18) is required only for insulators and semiconductors. In the case of slowpositron experiments, the time-independent source term $f_{i}(\mathbf{r})$ is equal to the implantation profile $P(z)$, which depends on the initial positron beam energy (cf. Sec. II.A.1).

The solutions $f(z)$ to the diffusion equation (18) have to obey certain boundary conditions. Firstly, the posi- 
tron density has to vanish in the material far away from the surface of incidence. Secondly, the positron current $-D_{+} \partial f(z) / \partial z+v_{d}(z) f(z)$ has to be continuous everywhere, especially through interfaces of different materials or through the sample surface. The positron current through an interface at $z=z_{0}$ and the positron density at that interface are related as

$$
-D_{+}\left(\frac{\partial f(z)}{\partial z}\right]_{\mid z=z_{0}}+v_{d}(z) f(z)=-v f\left(z_{0}\right),
$$

where $v$ is the escape rate through the interface. If the interface is totally absorbing, i.e., $v=\infty$, the boundary condition is that the positron density has to vanish at the surface: $f\left(z_{0}\right)=0$.

In order to model the time-resolved slow-positronbeam experiments (Lynn et al., 1984; Schödlbauer et al., 1988), the diffusion equation has to be solved in the time-dependent form. For a system with a homogeneous defect profile, an analytic solution can be found by the Green's-function method (Britton, 1991). It reads

$$
f(z, t)=\int_{0}^{\infty} G(z \mid x, t) P(x) d x,
$$

where $G(z \mid x, t)$ is the Green's function

$$
\begin{aligned}
G(z \mid x, t)=e^{-\lambda_{\mathrm{eff}} t}\left\{\frac{1}{\sqrt{4 \pi D_{+} t}}\left[e^{-(x-z)^{2} / 4 D_{+} t}+e^{-(x+z)^{2} / 4 D_{+} t}\right]\right. \\
\left.-\frac{v}{D_{+}} e^{\left(v / D_{+}\right)(x+z+v t)} \operatorname{erfc}\left[\frac{x+z}{\left(4 D_{+} t\right)^{1 / 2}}+v\left[\frac{t}{D_{+}}\right]^{1 / 2}\right]\right\} .
\end{aligned}
$$

Britton (1991) used the appropriate Green's function and solved for the time-dependent distribution $f(z, t)$. Then he determined the bulk positron fraction $f_{b}(t)$ by integration over $z$. Furthermore, the fraction $f_{s}(t)$ of positrons at a surface state and the parapositronium fraction $f_{p s}(t)$ were obtained using rate equations (similar to those for trapping into vacancies in bulk; see Sec. II.C.1) in which the different branching ratios and lifetimes were as parameters. Thereafter Britton calculated the annihilation rate as

$$
N(t)=\lambda_{b} f_{b}(t)+\lambda_{s} f_{s}(t)+\lambda_{p s} f_{p s}(t),
$$

where $\lambda_{b}, \lambda_{s}$, and $\lambda_{p s}$ are the annihilation rates for bulk, surface state, and parapositronium, respectively. Britton found that the resulting annihilation rates $N(t)$ could be described by a sum of two exponential components. The longer component corresponds to the absence of thermal desorption from the surface state (see Secs. II.C.6 and III.C), whereas the shorter one is due to the combined effects of positron annihilation in bulk and the fast parapositronium annihilation. Britton also studied the effects due to epithermal positrons and concluded that their role was not very important.

The diffusion-equation approach is frequently used in analyzing the results from the slow-positron-beam experiments (van Veen et al., 1990). The measured spectrum can be the fraction of reemitted positrons or Ps atoms. These fractions are proportional to the back-diffusion current on the surface. An alternative method is to measure the Doppler line-shape parameter $(S)$ of the positron annihilation radiation as a function of implantation energy. The $S$ parameter depends on the stationary positron distribution $f\left(z, E_{i}\right)$, on the defect profile, and on the annihilation rates in defect-free bulk, at defects, and at the surface. The annihilation rates reflect, in turn, the electronic structure at the annihilation site. In the analysis, one tries to find estimates for the actual defect profile. Simple functions such as a piecewise constant profile or a sum of a few Gaussian functions have often been used. The diffusion equation is solved, after which a theoretical spectrum is constructed. The parameters of the model defect profile are varied until the best fit with the measured spectrum is obtained. For this kind of analysis it is also important that one be able to determine independently the positron diffusion coefficient $D_{+}$.

\section{Positron diffusion coefficient}

The theoretical determination of the positron diffusion coefficient can be based on the relaxation-time approximation in Eq. (17). For the metals the acoustic longitudinal phonon scattering dominates, and its contribution can be calculated using the deformation-potential approximation (Bardeen and Shockley, 1950) in which the relaxation time is

$$
\tau_{p h}=\left(\frac{8 \pi}{9}\right)^{1 / 2} \frac{\hbar^{4} s}{\left(m^{*}\right)^{3 / 2}\left(k_{B} T\right)^{3 / 2} \gamma},
$$

and [cf. Eq. (4) for the positron-acoustic-phonon coupling constant]

$D_{+} \equiv \tau_{p h} \frac{k_{B} T}{m^{*}}=\left(\frac{8 \pi}{9}\right)^{1 / 2} \frac{\hbar^{4}\left\langle c_{i i}\right\rangle}{\left(m^{*}\right)^{5 / 2}\left(k_{B} T\right)^{1 / 2} E_{d}^{2}}$.

The theoretical $T^{-1 / 2}$ temperature dependence of the diffusion coefficient has also been observed with small deviations in slow-positron-beam experiments for several metals (Soininen et al., 1990). The reasons for these deviations are somewhat unclear. There may also be contributions from scattering mechanisms other than longitudinal-acoustic phonons, or the effective positron mass or the elastic constants may have a temperature 
dependence, which affects the results. For example, in the case of semiconductors and polar materials, one expects important contributions from optical phonons (Soininen et al., 1992).

The effective positron mass $m^{*}$ is usually larger than the free-positron mass. Typical estimates are of the order of 1.3-1.7m (see, e.g., Schultz and Lynn, 1988). Three phenomena affect the magnitude of the effective mass. The largest contribution is due to phonon scattering (Mikeska, 1967), which results in an asymmetric broadening of the positron momentum distribution. Secondly, the screening of positrons by electrons increases the effective mass. The third source is the normal effect due to the periodic lattice, i.e., the band mass. The effect of phonons on the positron diffusion coefficient thus comes through two routes. Firstly, they induce the scattering among different positron Bloch states, and, secondly, they increase the positron effective mass. The second point means that the unit, which performs scattering, is a quasiparticle in an interacting many-body system, rather than a single, bare positron.

\section{Positron trapping into defects}

At open-volume defects (such as monovacancies and larger vacancy clusters, possibly decorated by impurity atoms) in solids, the potential sensed by the positron is lowered due to the reduction in the repulsion by the positive-ion cores. As a result, a localized positron state at the defect can have a lower energy eigenvalue than a state delocalized over the lattice. The transition from the delocalized state to the localized one is called positron trapping. In this transition the energy difference between the initial and final positron states, the trapping energy, is transferred to the host solid. Besides the vacancy-type defects, other defects capable of trapping positrons include such open-volume defects as dislocations (Häkkinen et al., 1989), in the core regions of which the ion density is reduced, and negatively charged defects in semiconductors or insulators such as negative impurity ions (Saarinen et al., 1990). The binding energies of positrons to these latter kinds of defects, so-called shallow traps, are relatively small. Therefore these traps are important only at low temperatures in which the thermally activated escape, called detrapping, from the shallow traps is not possible. In experiments the trapping of positrons into defects is monitored through the changes in the annihilation parameters, such as the positron lifetime or the width of the annihilation radiation line. The theoretical estimation of the annihilation parameters is discussed in Sec. III. In this section we discuss the kinetic and thermodynamic aspects of positron trapping.

\section{Positron trapping model}

In the positron lifetime spectroscopy for defects in solids, the lifetime spectra are fitted, after source and background corrections, to a sum of exponentially decaying components. Due to difficulties of statistical origin, it is usually possible to use only two components, although the physical situation (number of different types of traps) would justify the use of more components. In the twocomponent fit, the model function is the derivative of the number of positrons with respect to the time the positrons have lived in the sample, i.e.,

$$
\frac{d N(t)}{d t}=-N_{0}\left[\frac{I_{1}}{\tau_{1}} e^{-t / \tau_{1}}+\frac{I_{2}}{\tau_{2}} e^{-t / \tau_{2}}\right]
$$

convoluted with a function describing the resolution of the spectrometer. Here, $N_{0}$ is the integral of the model function over time, i.e., the total number of counts in the source- and background-corrected spectrum, $\tau_{1}$ and $\tau_{2}$ are the apparent lifetimes, and $I_{1}$ and $I_{2}$ are the corresponding relative intensities.

The decomposition of the positron lifetime spectrum is explained by the trapping model (Bertolaccini et al., 1971; Brandt, 1974), which gives the rate equations for the positrons annihilating in delocalized states (as free positrons) and in localized states (as trapped positrons). If there is only one type of defect in the sample, the kinetic equations are written as

$$
\begin{aligned}
& \frac{d f_{b}}{d t}=-\lambda_{b} f_{b}-\kappa f_{b}+f_{i}, \\
& \frac{d f_{t}}{d t}=-\lambda_{t} f_{t}+\kappa f_{b},
\end{aligned}
$$

where $f_{b}\left(f_{t}\right)$ is the probability that the positron will be free (trapped) at time $t . \lambda_{b}$ and $\lambda_{t}$ are the annihilation rates for free and trapped positrons, respectively. $f_{i}$ is the source term, i.e., the number of positrons entering the system per unit time. In the kinetic trapping model, the positron trapping rate $\kappa$ is proportional to the defect concentration $c_{t}$ in the sample,

$$
\kappa=v c_{t},
$$

This defines the trapping coefficient $v$ (the trapping rate for a unit atomic concentration of defects; $\left.[v]=s^{-1}\right)$. It is the defect analog of the escape rate through a (planar) surface in Eq. (23). Equation (30) is directly obtained from the time-dependent diffusion equation (15) by omitting the spatial dependence of the positron distribution and assuming a constant source term. Equation (31) is then the corresponding equation for the trapped positrons.

The trapping rate $\kappa$, the positron bulk lifetime $\tau_{b}=1 / \lambda_{b}$, and the lifetime at the defect $\tau_{t}=1 / \lambda_{t}$ defined through the trapping model are related to the twocomponent fitting parameters in Eq. (29) as (West, 1979)

$$
\frac{1}{\tau_{1}}=\frac{1}{\tau_{b}}+\kappa,
$$$$
\tau_{2}=\tau_{t}
$$ 


$$
I_{2}=1-I_{1}=\frac{\kappa}{\kappa+\lambda_{b}-\lambda_{t}}
$$

It is easy to generalize the trapping model, Eqs. (30) and (31), to include several types of traps and detrapping.

The experimental determination of the trapping coefficient is difficult, because the absolute defect concentrations are usually not well known. The experimental determination is possible by the simultaneous measurement of the two-component positron lifetime spectrum and the vacancy concentration. An example of this would be the differential-dilatometric method as a function of temperature (Kluin and Hehenkamp, 1991; Hehenkamp et al., 1992).

It is generally accepted that the trapping coefficients for vacancies in metals are of the order of $10^{14}-10^{15} \mathrm{~s}^{-1}$ (Nieminen and Manninen, 1979; West, 1979). The magnitude of the trapping coefficient is about five orders of magnitude larger than the typical positron annihilation rates $\left(\lambda_{b} \approx 5 \times 10^{9} \mathrm{~s}^{-1}\right)$. This makes it possible to detect vacancy concentrations of the order of $10^{-7}-10^{-4}$. The upper limit is due to the saturation of the trapping: the lifetime spectrum is totally dominated by the defect component $\tau_{t}$, and it is not possible to determine the trapping fraction. The experimental and theoretical estimations of the values of the trapping coefficient are important because they are needed to extract defect formation energies and concentrations from the positron lifetime measurements. Moreover, in the case of defects in semiconductors, the temperature dependence of the trapping rate can be used to identify the charge state of the defect.

\section{Positron trapping coefficient}

An important goal of the theoretical work on the positron trapping process has been the estimation of the trapping coefficient. Of special interest has been the dependence of the trapping coefficient on the electronic and atomic structure of the defect, on the host temperature (which determines the energy distribution of the thermalized positrons in the initial state), and on the positron energy in the initial, delocalized (nonthermalized) state (resonance trapping). In the case of vacancies or small vacancy clusters, positron trapping is transition limited. This means, in a semiclassical picture, that the positron diffusion to the defects does not limit the trapping rate, but that the rate is determined by the quantummechanical probability of the transition from the delocalized state to the localized one. This is manifest in the kinetic trapping model of Eqs. (30) and (31). In the transition-limited regime, the positron trapping coefficient is directly the transition rate between the delocalized and localized states is given by Fermi's golden rule. The golden rule was first used in the context of positron trapping by Hodges (1970).

The transition rate depends not only on the initial- and final-state positron wave functions, but also on the nature of the process by which the trapping energy is transferred to the host. For vacancy-type defects in metals, electron-hole excitation is the most important. In this case the golden rule reads (Hodges, 1970)

$$
v(p)=\frac{4 \pi}{\hbar} \sum_{\mathbf{k}} \sum_{\mathbf{q}}|M(\mathbf{p}, \mathbf{q})|^{2} f_{F}^{0}\left(\frac{\hbar^{2} \mathbf{k}^{2}}{2 m_{e}}, 0\right]\left[1-f_{F}^{0}\left[\frac{\hbar^{2}(\mathbf{k}+\mathbf{q})^{2}}{2 m_{e}}, 0\right]\right] \delta\left(\epsilon_{i}-\epsilon_{f}\right)
$$

Above, $M(\mathbf{p}, \mathbf{q})$ is the matrix element between the initial $\left(\psi_{i}\right)$ and the final $\left(\psi_{f}\right)$ electron-positron states,

$M(\mathbf{p}, \mathbf{q})=\int d \mathbf{r} \int d \mathbf{r}^{\prime} \psi_{i}^{*}\left(\mathbf{r}, \mathbf{r}^{\prime}\right) V\left(\mathbf{r}-\mathbf{r}^{\prime}\right) \psi_{f}\left(\mathbf{r}, \mathbf{r}^{\prime}\right)$,

where $\mathbf{r}$ and $\mathbf{r}^{\prime}$ refer to the positron and electron coordinates, respectively. $V$ is the screened Coulomb interaction between the electron and positron. In Eq. (36) the terms on the right-hand side of the matrix element take care of the momentum and energy conservations in the process. More specifically, $\mathbf{p}$ is the momentum of the initial positron state, $\mathbf{k}$ is that of the electron before the excitation, and $\mathbf{q}$ is the momentum transfer. $\epsilon_{i}$ and $\epsilon_{f}$ refer to the initial- and final-state energies, respectively. If the initial and final electron states are approximated as plane waves, the matrix element reduces by a Fourier transform to

$$
M(\mathbf{p}, \mathbf{q})=\frac{1}{\Omega} V(\mathbf{q}) \int d \mathbf{r}\left(\psi_{i, \mathbf{p}}^{+}\right)^{*}(\mathbf{r}) \psi_{f}^{+}(\mathbf{r}) e^{i \mathbf{q} \cdot \mathbf{r}}
$$

where $\psi_{i, \mathrm{p}}^{+}$and $\psi_{f}^{+}$are the initial and final positron states, respectively, and $\Omega$ is the normalization volume for the electron states (plane waves). This equation for the trapping rate corresponds to Eq. (3) for the positron scattering off conduction electrons. Because the momentum and energy transfers may be large in the trapping process, the low-momentum limit cannot be taken. Therefore the screened Coulomb interaction must be calculated, for example, in the Thomas-Fermi approximation

$$
V(\mathbf{q})=\frac{4 \pi e^{2}}{q^{2}+q_{T F}^{2}}
$$

where $q_{T F}$ is the Thomas-Fermi screening length. On the other hand, the zero-temperature Fermi distribution can be used.

If the positron binding energy $E_{b}$ to the defect is small, of the order of $0.1 \mathrm{eV}$ or less, a trapping process in which the energy is transferred to a single phonon becomes important. For higher energies, one-phonon processes are not possible, due to the Debye cutoff, and the probabilities for multiphonon processes are very small. In the case of longitudinal-acoustic phonons, the formula for the trapping coefficient is 


$$
v(p)=\left.\frac{4 \pi}{\hbar} \sum_{\mathbf{q}}|| M(\mathbf{p}, \mathbf{q})\right|^{2} \delta\left(\frac{p^{2}}{2 m^{*}}+E_{b}-\hbar s q\right)\left[f_{B}^{0}(\hbar s q, T)+1\right],
$$

with

$$
M(\mathbf{p}, \mathbf{q})=\frac{\gamma(\hbar q / \Omega)^{1 / 2}}{1+q^{2} / q_{T F}^{2}} \int d \mathbf{r} \psi_{i, \mathbf{p}}^{+*}(\mathbf{r}) \psi_{f}^{+}(\mathbf{r}) e^{i \mathbf{q} \cdot \mathbf{r}}, \quad \gamma=\frac{E_{d}}{(2 \rho s)^{1 / 2}} .
$$

Here Thomas-Fermi screening and the deformationpotential approximation are used, and the positronphonon coupling constant $\gamma$ is as in Eq. (5). The above equation corresponds to Eq. (4) for positron scattering off phonons, but here the low momentum limit is not taken.

The temperature dependence of the positron trapping coefficient is, especially in the case of semiconductors, an important issue in experimental defect studies. The temperature dependence gives information about the character of the defects, such as the charge state of the defect. The temperature dependence of the trapping coefficient arises mainly from the temperature dependence of the energy distribution of the thermalized positrons before trapping, because in many cases the transition rate depends strongly on the positron energy in the initial state. In the case of phonon excitations, the transition rate also depends on temperature through the thermal distribution of the phonons. In order to obtain the temperature dependence of the trapping coefficient, the initial positron momentum- (or energy-) dependent coefficient in Eqs. (36) and (40) has to be averaged over the energy distribution of the thermalized positrons. The MaxwellBoltzmann distribution gives the trapping coefficient for temperature $T$,

$$
\begin{gathered}
v(T)=\frac{2}{\pi^{1 / 2}\left(k_{B} T\right)^{3 / 2}} \int_{0}^{\infty} d E v\left(p=\sqrt{2 m^{*} E}\right) \\
\times e^{-E / k_{B} T} \sqrt{E} .
\end{gathered}
$$

In the calculations for the positron trapping coefficients, the initial and final electron-positron states have been described as products of independent electron and positron wave functions. Moreover, it has been necessary to approximate the electron and positron states using rather simple models. As assumed also in Eq. (41), plane waves have usually been used for the initial and final electron states in the electron-hole excitation processes. The positron wave functions used have also been approximate. For example, Nieminen and Laakkonen (1979) described the trapped state by a spherically symmetric Gaussian function, and the delocalized state by a plane wave orthogonalized against the trapped state. Recently, McMullen and Stott (1986) used a model in which they solved the exact initial- and final-state positron wave functions for a spherical square-well potential describing a vacancy in metal. The delocalized positron wave functions in the spherical symmetry are scattering states

$$
\psi_{i, \mathbf{p}}^{+}(\mathbf{r})=\frac{4 \pi}{\sqrt{\Omega}} \sum_{l, m} i^{l} e^{i \delta_{l}} Y_{l}^{m *}(\hat{\mathbf{p}}) Y_{l}^{m}(\widehat{\mathbf{r}}) R_{l p}(r),
$$

where $Y_{l}^{m}$ are spherical harmonics and $R_{l p}$ are radial wave functions, which are solved for the trapping potential in question. The crucial property of the true scattering states is the appearance of the resonances for nonzero $l$ components of $\psi_{i, \mathrm{p}}^{+}$at certain energies. At the resonance the delocalized positron wave function is strongly enhanced at the defect, which can increase the positron trapping coefficient by orders of magnitude.

According to calculations by Nieminen and Laakkonen (1979), the positron trapping coefficient for small vacancy clusters in $\mathrm{Al}$ are of the order of $10^{14}-10^{15} \mathrm{~s}^{-1}$. This is in accordance with experimental estimations (for reviews, see Nieminen and Manninen, 1979 or West, 1979). The trapping coefficient increases with the size of the vacancy cluster until the electron phase space (simultaneous conservation of the momentum and energy in the electron-hole process) sets an upper limit. According to the model by Nieminen and Laakkonen (1979), the trapping coefficient for the vacancies and small vacancy clusters is temperature independent. In the case of large vacancy clusters, the trapping coefficient decreases with increasing temperature. This is because the positron thermal wavelength $\Lambda_{+}$becomes similar in size to the spatial extent of the defect. Mathematically, this means that the function in the integral for the matrix element [Eq. (41)] becomes oscillating for typical thermal energies.

\section{Resonance trapping}

The picture arising from the model of McMullen and Stott (1986) for positron trapping into small vacancy clusters is due to scattering resonances more complicated than those by Nieminen and Laakkonen (1979). Figure 12 shows the positron trapping coefficient calculated as a function of the energy of the delocalized positron. The radius of the square well corresponds to the Wigner-Seitz radius of $\mathrm{Al}$, and the depth of the well is varied. A very strong $p$-type resonance is pushed to lower energies when the depth of the well is increased. Puska and Manninen (1987) studied (among other things) the temperature dependence of the trapping coefficient in this model. They showed that, although the temperature dependence is usually weak, a resonance close to thermal energies may cause a very strong increase in the trapping 


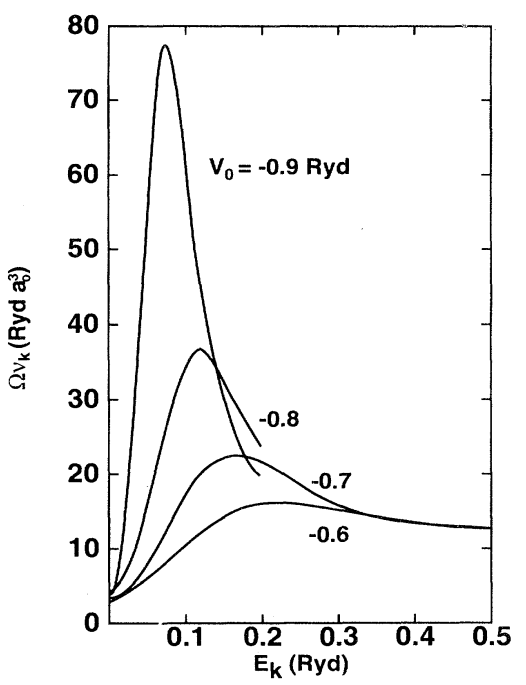

FIG. 12. Positron trapping coefficient for a vacancy as a function of the initial positron energy. The potential sensed by the positron at the vacancy is modeled by a spherical square well, the radius of which, $2.99 a_{0}$, corresponds to Al. The depth of the well is varied from 0.6 to $0.9 \mathrm{Ry}$ (from McMullen and Stott, 1986).

coefficient as a function of temperature. The relevance of this so-called resonance trapping to the measurable parameters has been discussed in several works (for more references, see Jensen and Walker, 1990). The model was used to explain the slow-positron-beam experiments by Nielsen et al. (1986), who reported an intensity depletion of epithermal (energy less than $1 \mathrm{eV}$ ) positrons reemitted from an Al sample containing defects. The calculations by Lynn et al. (1987) argue that resonance trapping can even explain quantitatively the experimental results. On the other hand, Jensen and Walker (1990) studied the effects of the prethermal trapping by solving the Boltzmann equation for the positron momentum during the thermalization process. They concluded that trapping at resonance energies is only a small fraction of the total trapping which occurs mainly at thermal energies. This is because a positron slowing down through the resonance region takes a short time compared to the time that a positron spends at thermal energies before trapping. Jensen and Walker (1990) argued further that prethermal trapping has then only a minor effect on the intensities of the different components in positron lifetime measurements.

\section{Positron trapping in semiconductors}

Electron-hole excitation in insulators or in semiconductors is essentially hindered by the forbidden energy gap between the valence and conduction bands. Howev$\mathrm{er}$, if the positron binding energy to the defect is larger than the band gap, or if there are defect-induced localized deep states lying in the band gap, electron-hole excitation is possible. The trapping coefficient may in these cases be of the same order as in metals, as was shown in the model calculations by Puska et al. (1990). In the following we shall discuss in detail the results and the models for positron trapping in semiconductors arising from these calculations.

Puska et al. (1990) used positron wave functions calculated for a spherical well potential mimicking a vacancy in $\mathrm{Si}$. The charged states were described by adding to the defect potential well a long-range Coulomb tail $Q / \epsilon r$, where $\epsilon$ is the zero-frequency dielectric constant and $Q$ is the charge of the defect (see Fig. 13). The Coulomb tail has to be cut off when it approaches the vacancy in order to mimic the weak localization of the extra charge. This results in a potential shift of $Q \times(0.1 \mathrm{eV})$ near the vacancy. For neutral vacancies in semiconductors, the results are also similar to those for metals in the sense that the trapping coefficient is nearly independent of temperature. The behavior of the trapping coefficient for the charged vacancies is, however, quite different. This is because (a) the initial positron states far away from the defect are now Coulomb waves instead of spherical waves, and because (b) the long-range Coulomb potential induces (in principle an infinite number of) bound Rydberg states. The square of the amplitude of the Coulomb wave has a maximum at the center of the vacancy, and it behaves as (Mott and Massey, 1987)

$$
\left|\psi_{i, \mathrm{p}}^{+}(0)\right|^{2} \propto \frac{\alpha}{e^{2 \pi \alpha}-1},
$$

where
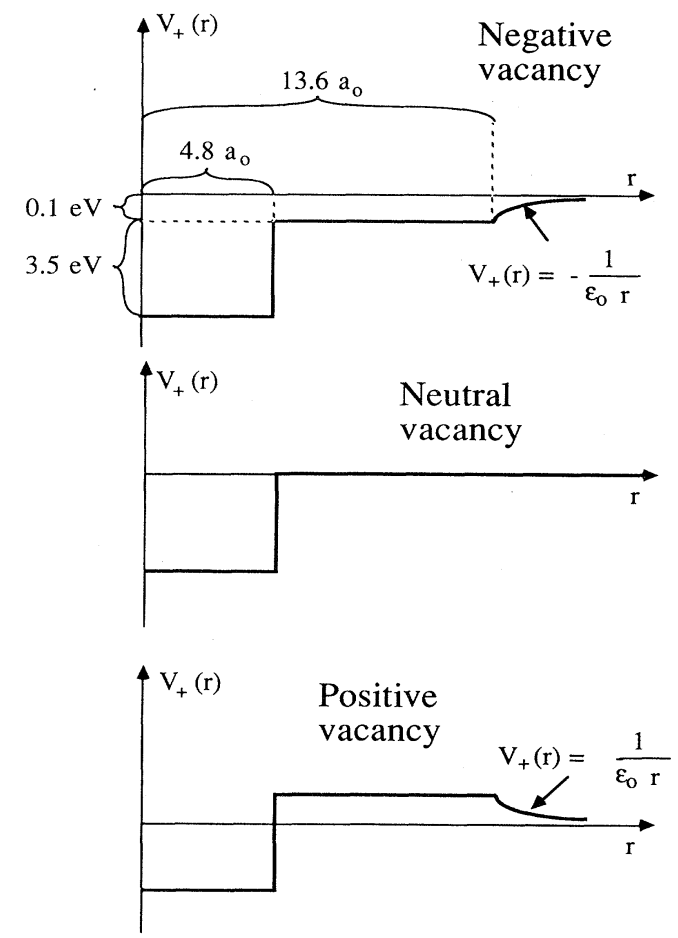

FIG. 13. Positron-model potentials for singly negative, neutral, and singly positive Si vacancy (from Puska et al., 1990). 


$$
\alpha=\frac{m_{+} Q}{\epsilon p},
$$

and $p$ is the positron momentum. For a negative charge state $Q<0$, the square of the matrix element [Eq. (37) or (41)] and the ensuing positron trapping coefficient are inversely proportional to the square root of the positron energy. When the average over the positron initial energies is calculated, the integral in Eq. (42) becomes proportional to temperature and, consequently, due to the prefactor proportional at $T^{-3 / 2}$, the resulting trapping coefficient depends on temperature as $T^{-1 / 2}$. Equations (44) and (45) explain the behavior of the trapping coefficient in the case of neutral and positively charged vacancies, too. Neutral vacancies do not produce a trapping coefficient diverging at low temperatures because, for $\alpha$ values close to zero, the amplitude in Eq. (44) approaches a constant value and the wave function becomes a plane wave. For a positively charged vacancy, the square of the initial positron wave function is due to the exponential function's being vanishingly small, and therefore the trapping coefficient becomes orders of magnitude smaller than for neutral or negatively charged vacancies.

The picture based on Eq. (44) is reproduced in the results of numerical model calculations (Puska et al., 1990). The positron trapping rates obtained for singly positive, for neutral, and for singly and doubly negative vacancies are shown in Fig. 14 as a function of temperature. In these results, the process liberating the positron binding energy is the excitation of an electron from a deep level to the conduction band. The trapping coefficient for the positively charged vacancy increases as temperature arises, because the probability of tunneling through the Coulomb barrier increases. However, even for the highest temperatures shown, the ensuing trapping rate is small compared to the positron annihilation rate, and trapping to positive vacancies is not important. According to Fig. 14 the trapping coefficient for a singly

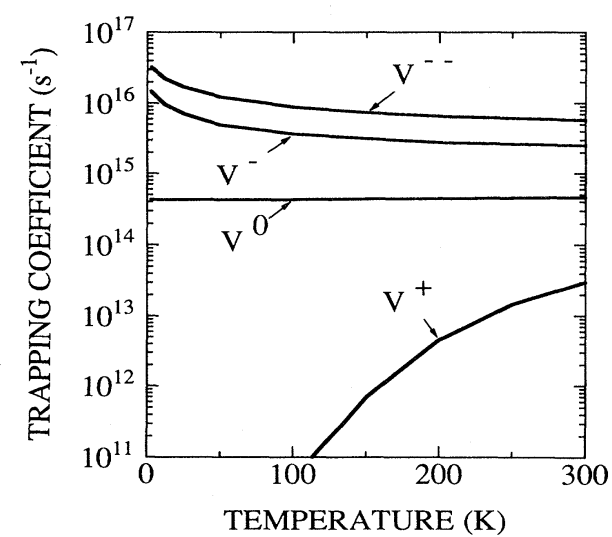

FIG. 14. Temperature dependence of the positron trapping coefficient for singly positive $\left(V^{+}\right)$, neutral $\left(V^{0}\right)$, singly negative $\left(V^{-}\right)$, and doubly negative $\left(V^{--}\right)$vacancies. The process of electron excitation from a deep level to the conduction band was used (from Puska et al., 1990). negative vacancy is, at room temperature, about an order of magnitude larger than that for the neutral vacancy. The difference between the results for the singly and doubly negative vacancies can be explained by the chargestate $(Q)$ dependence in Eq. (44) and by the number of electrons in the initial localized state.

The predicted $T^{-1 / 2}$ dependence of the positron trapping coefficient $v$ was recently seen in experiments. The trapping rate $\kappa$ to vacancy-type defects obtained by analyzing the positron lifetime spectra from protonirradiated $\mathrm{Si}$ obeyed this power law over a wide temperature region from 15 to $150 \mathrm{~K}$ (S. Mäkinen et al., 1990). Electron-irradiated pure $\mathrm{Si}$ and $\mathbf{P}$-doped $\mathrm{Si}$ also showed this dependence below the temperature of $50 \mathrm{~K}$ (Mäkinen, Hautojärvi, and Corbel, 1992). Very recently, the capture of holes at acceptors in Ge was explained with a cross section that is inversely proportional to temperature (Darken, 1992). This corresponds to a trapping rate proportional to the inverse square root of temperature [see Eq. (54)]. Thus the $T^{-1 / 2}$ dependence seems to be a general result for the capture of charge carriers in semiconductors.

The long-range Coulomb potential sensed by the positron at negatively charged defects in semiconductors induces Rydberg states, which are weakly bound and therefore spatially relatively extended. The existence of the Rydberg states affects the positron trapping phenomenon. This was also shown by the model calculations for vacancies in Si (Puska et al., 1990). Because of the large extension, the transition rates for positrons from the delocalized (Coulomb) states to the Rydberg states may be rather high. The calculations also show that the transition from a Rydberg state to the ground state at the vacancy is usually fast enough (faster than the positron annihilation rate) so that the total trapping rate $\kappa$ monitored in the measurements can be determined by the trapping coefficient to the Rydberg state. Therefore the total trapping rate can be essentially higher than that for metal vacancies. Because the binding energies at Rydberg states are small, of the order of $0.1 \mathrm{eV}$, the transition from delocalized states to the Rydberg states proceeds mainly via phonon emission. The following transition to the ground state may take place as an excitation of an electron from the deep levels or from the valence band. The model calculations show that transitions between the Rydberg states are so slow that they cannot effectively take place within the positron lifetime. Therefore the cascade model (Lax, 1960), in which the charged particle relaxes down in energy following successive Rydberg states, and which had been used earlier for the recombination of the charge carriers, cannot be adapted for positrons. Recently, the cascade process was also shown to be doubtful for hole capture at acceptors in Ge (Darken, 1992).

In order to describe the temperature dependence of the positron trapping rate in the presence of the Rydberg states, one needs a dynamic model, because thermal detrapping from the shallow Rydberg states is important 
even at relatively low temperatures. The situation is depicted in Fig. 15(a). The annihilation rates for the delocalized state and the shallow Rydberg states are essentially equal $\left(\lambda_{b}\right)$, and that for the ground state at the vacancy is $\lambda_{t}<\lambda_{b}$. For a given vacancy concentration $c_{v}$, transitions from the delocalized states to a Rydberg state take place with the trapping rate $\kappa_{R}$, from the Rydberg state to the final trap state with the rate $\eta_{R}$, and from the delocalized state directly to the ground state with the trapping rate $\kappa$. The positrons escape due to thermal activation from the Rydberg state with the rate $\delta_{R}$, but do not escape from the tightly bound ground state. When the populations of the delocalized and localized states are in thermal equilibrium, the detrapping rate $\delta_{R}$ and the trapping rate $\kappa_{R}$ into the Rydberg state are related as (Manninen and Nieminen, 1981)

$$
\frac{\delta}{\kappa}=\frac{1}{c_{v}}\left[\frac{m+k_{B} T}{2 \pi \hbar^{2}}\right]^{3 / 2} e^{-E_{R} / k_{B} T},
$$

where $E_{R}$ is the positron binding energy in the Rydberg state.

The above dynamic model for the positron trapping to vacancy-type defects in semiconductors is analogous to the model presented by Smedskjaer et al. (1980) for positron trapping into jogs or kinks at dislocation lines in metals. In that model the weakly bound positron state delocalized along the dislocation line is the precursor state, and the open volume at a point defect serves as the final trap. The occupations of the different states in both models (here $n_{b}, n_{r}$, and $f_{t}$ ) can be obtained from steady-state rate equations (Smedskjaer et al., 1980). The parameters of the model in Fig. 15(a) can be related to the trapping rate (below $\kappa^{\prime}$ ) of the two-state trapping model shown in Fig. 15(b) and used in analyzing the positron lifetime spectra. If one replaces the series of Rydberg states with one effective state and assumes that $\eta_{R} \gg \lambda_{b}$ and $\kappa \ll<\lambda_{b}$, one obtains (Puska et al., 1990)

$$
\kappa^{\prime}=\frac{\eta_{R} c_{v} \kappa_{R}}{\eta_{R} c_{v}+\kappa_{R}\left(m+k_{B} T / 2 \pi \hbar^{2}\right)^{3 / 2} e^{-E_{R} / k_{B} T}} .
$$

According to this equation the temperature dependence of $\kappa^{\prime}$ arises from two effects:

(a) The positron trapping coefficient $\kappa$ into a Rydberg state depends on temperature. The dependence is close to the " $T^{-1 / 2}$ law" because the initial state is a Coulomb wave; but $\kappa$ also has a weaker temperature dependence because the binding energy is of the same order as the positron thermal energy (Puska et al., 1990). The temperature dependence due to $\kappa$ dominates at very low temperatures.

(b) When temperature increases, detrapping from the Rydberg state (the exponential function in the denominator) becomes important. This results in a trapping rate $\kappa^{\prime}$ which decreases more rapidly as $T^{-1 / 2}$. As an example, Fig. 16 shows the trapping rate deduced from positron lifetime measurements of electron-irradiated pure and $\mathbf{P}$ doped Si (Mäkinen, Hautojärvi, and Corbel, 1992). The experimental results are fitted to the model of Eq. (47), and good agreement is obtained with reasonable values of the fitting parameters $\kappa_{R}, \eta c_{v}$, and $E_{R}$. Below $\sim 50 \mathrm{~K}$, $\kappa^{\prime} \approx \kappa$ and a simple $T^{-\alpha}$ dependence is assumed with $\alpha$ slightly less than 0.5 . Above $50 \mathrm{~K}$ the behavior switches to the exponential decay due to detrapping. The same temperature dependence of $\kappa$ is used at both low and high temperatures.

\section{Positron trapping at voids in metals}

In the case of spatially large defects, such as voids in metals, the positron trapping coefficient may become so large (due to the large density of the final states) that the positron mobility (diffusion to the defect) starts to limit the total trapping rate. The switching from transitionlimited to diffusion-limited trapping was first studied by McMullen (1977, 1978) using a quantum-mechanical theory based on positron-phonon scattering. In the case of spatially extended defects, the diffusion theory can be employed in describing the behavior of the trapping rate (Brandt, 1974; Nieminen et al., 1979). According to the resulting picture, the trapping rate is decreased because the positron density is depleted near the defect due to the trapping phenomenon itself.

Nieminen et al. (1979) have presented a comprehen-

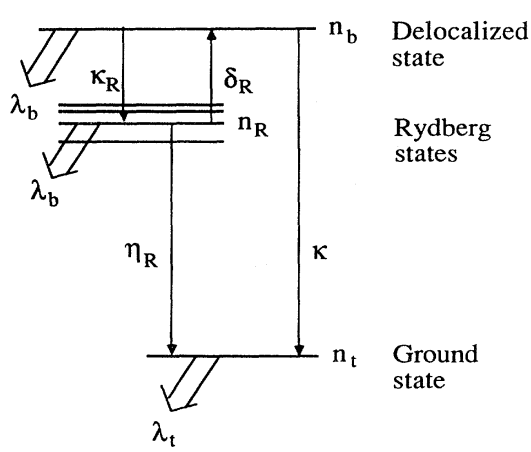

(a)

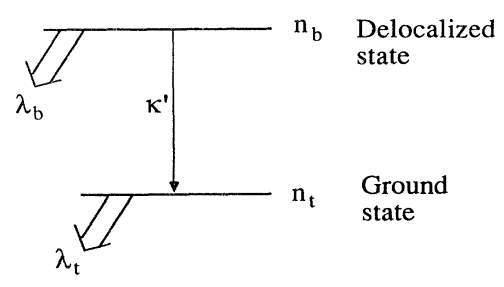

(b)
FIG. 15. Positron trapping into a negative vacancy in a semiconductor. (a) general schematic view; (b) two-state positron trapping model (from Puska et al., 1990). 


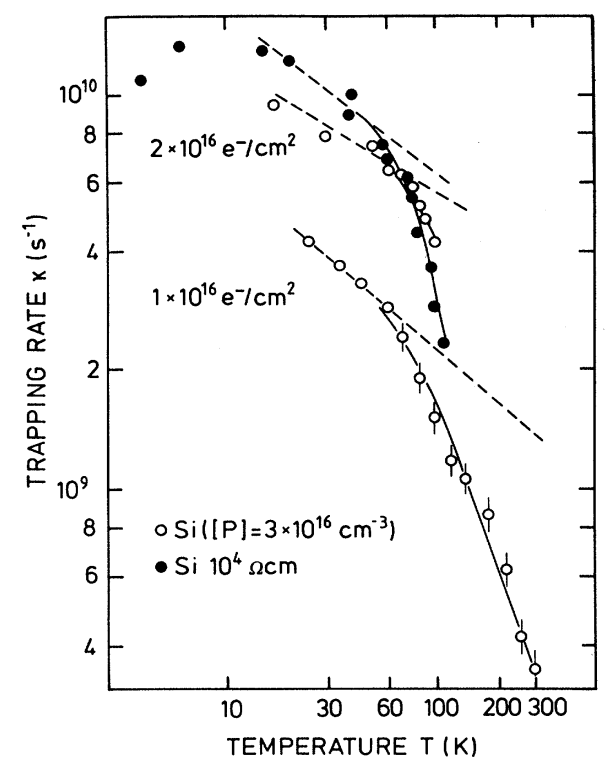

FIG. 16. Temperature dependence of the positron trapping rate in electron-irradiated Si. The defects are identified as the isolated negative vacancy and the negative phosphorus-vacancy pair in high-resistivity and $P$-doped $\mathrm{Si}$, respectively. The dashed lines correspond to the $T^{-\alpha}$ dependence for $\kappa$, and the solid line is the fit using the functional form of Eq. (47). From Mäkinen, Hautojärvi, and Corbel, 1992.

sive semiempirical theory for positrons in a metal containing a finite concentration of voids. A similar approach for positron trapping at grain boundaries in metals was recently adopted by Dupasquier et al. (1993). The theory by Nieminen et al. (1979) gives, among other things, the intensity $I_{2}$ of the void component in the two-component trapping model. This can be directly used in the analysis of the lifetime spectra measured as a function of temperature. Firstly, Nieminen et al. (1979) make the Wigner-Seitz approximation; i.e., they define a Wigner-Seitz sphere, the radius $R_{v}$ of which is determined by the void concentration $c_{v}$ :

$$
R_{v}=\left(\frac{3}{4 \pi c_{v}}\right)^{1 / 3} \text {. }
$$

Concentric with this sphere is a void sphere with radius $r_{v}$.

The density of free positrons $f_{b}(r, t)$ (in the region $r_{v} \leq r \leq R_{v}$ ) is a function of time and distance from the common center of the spheres. The spatial dependence of the trapped positrons is not necessary to the model, and therefore only the number $f_{t}(t)$ of trapped positrons at time $t$ is used. The model employs the picture in which the fate of a single positron is followed, and the positron distributions are then obtained from the diffusion-annihilation equations

$$
\frac{\partial f_{b}(r, t)}{\partial t}=D_{+} \nabla^{2} f_{b}(r, t)-\lambda_{b} f_{b}(r, t)
$$

and

$$
\frac{\partial f_{t}(t)}{\partial t}=-\lambda_{t} f_{t}(t)+v f_{b}(r, t)_{\mid r=r_{v}},
$$

where $\lambda_{b}$ and $\lambda_{t}$ are the annihilation rates for free and trapped positrons, respectively. $v$ is the trapping rate per unit concentration of voids $\left([v]=\mathrm{cm}^{3} \mathrm{~s}^{-1}\right)$. In the diffusion equation (49) the source and trapping terms appearing in Eq. (15) are replaced with the following boundary and initial conditions for the positron distribution $f_{b}(r, t)$,

$$
\begin{aligned}
& {\left[\frac{\partial f_{b}(r, t)}{\partial r}\right]_{\mid r=R_{v}}=0,} \\
& D_{+} 4 \pi r_{v}^{2}\left[\frac{\partial f_{b}(r, t)}{\partial r}\right]_{\mid r=r_{v}}=v f_{b}(r, t)_{\mid r=r_{v}}, \\
& f_{b}(r, 0)=n_{0} \text { when } r_{v} \leq r \leq R_{v}, \\
& f_{b}(r, 0)=0 \text { otherwise. }
\end{aligned}
$$

Moreover, the distribution of the trapped positrons obeys the initial condition

$$
f_{t}(0)=0 \text {. }
$$

The first of the conditions for free positrons [Eq. (51)], the Wigner-Seitz condition, means that the positron density "flows" in the trapping process with an equal probability to all voids, the concentration of which is $c_{v}$. The next equation (52) gives the flux of positrons through the void surface.

The solution (Carslaw and Jaeger, 1959) of Eq. (49) can be expressed as a sum of $r$-dependent eigenfunctions $f_{n}(r)$, each of which decays with a characteristic time constant $D_{+} \alpha_{n}^{2}+\lambda_{b}$

$$
f_{b}(r, t)=\sum_{n=1}^{\infty} e^{-\left(D_{+} \alpha_{n}^{2}+\lambda_{b}\right) t} f_{n}(r)
$$

After a short time from $t=0$ (typically $<5 \mathrm{ps),} \mathrm{only} \mathrm{the}$ longest living of the eigenfunctions for the positron density $f_{b}(r, t)$ survives. The so-called quasiequilibrium state, in which the form of the positron density stops changing (but the magnitude decays), has been reached. Figure 17 gives typical quasiequilibrium positron densities at different temperatures. It can be seen that at low temperatures the positron density is rather uniform, reflecting the large positron mobility (or diffusion coefficient). Towards higher temperatures, the positron diffusion coefficient decreases due to phonon scattering, and the free positron density becomes strongly depressed at the void surface due to the trapping. It is said that the positron trapping is then diffusion limited.

The fraction of trapped positrons is obtained from the solution $f_{b}(r, t)$ via the flux [Eq. (52)] at the void surface $r=r_{v}$ : 


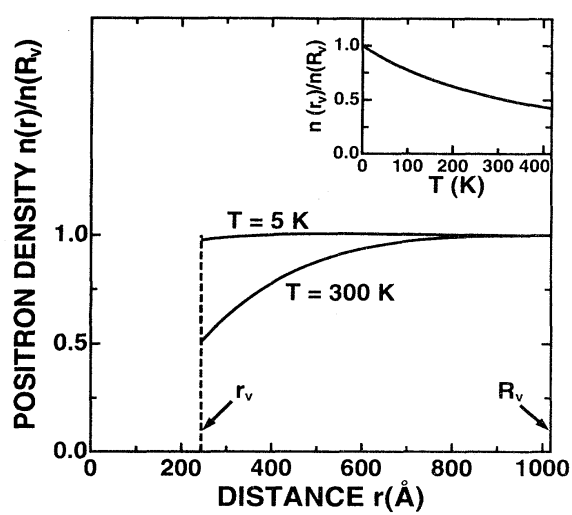

FIG. 17. Quasiequilibrium positron densities near a void in a metal (from Nieminen et al., 1979).

$$
\begin{aligned}
F & =\frac{R_{v}^{3}}{R_{v}^{3}-r_{v}^{3}} \frac{v c_{v}}{n_{0}} \int_{0}^{\infty} d t f_{b}\left(r_{v}, t\right) \\
& =\frac{R_{v}^{3}}{R_{v}^{3}-r_{v}^{3}} \frac{v c_{v}}{n_{0}} \sum_{0}^{\infty} \frac{f_{n}\left(r_{v}\right)}{\lambda_{b}+D_{+} \alpha_{n}^{2}} .
\end{aligned}
$$

Above, $R_{v}^{3} /\left(R_{v}^{3}-r_{v}^{3}\right)$ is a geometrical factor which enters because the free positrons are initially confined within the "interstitial" region $r_{v} \leq r \leq R_{v}$. The parameter $I_{2}$ corresponding to the two-component analysis of the positron lifetime spectra can be obtained by using Eq. (50). The result is

$$
I_{2}=\frac{R_{v}^{3}}{R_{v}^{3}-r_{v}^{3}} \frac{v c_{v}}{n_{0}} \sum_{0}^{\infty} \frac{f_{n}\left(r_{v}\right)}{\lambda_{b}-\lambda_{t}+D_{+} \alpha_{n}^{2}} .
$$

The temperature dependence of the fraction $F$ of trapped positrons and the intensity $I_{2}$ arises through the diffusion coefficient $D_{+}$and through the trapping rate $v$. If the diffusion is limited by scattering from longitudinal-acoustic phonons, $D_{+}$decreases as $T^{-1 / 2}$ when temperature increases [Eq. (28)]. Nieminen et al. (1979) describe the temperature dependence of $v$ semiclassically. The trapping rate per unit concentration of voids is written as $\left([v]=\mathrm{cm}^{3} \mathrm{~s}^{-1}\right)$

$$
v=v_{+} \sigma=\left(\frac{3 k T}{m^{*}}\right)^{1 / 2} \sigma=\frac{\hbar p}{m^{*}} \sigma,
$$

where $v_{+}$and $p$ are the thermal velocity and momentum of positrons, respectively. $\sigma$ is a positron capture cross section of the void. It can be approximated by the $s$-wave part only (Nieminen et al., 1979):

$$
\sigma=\pi\left(r_{v}+\Lambda_{+}\right)^{2} \xi=\pi\left(r_{v}+\Lambda_{+}\right)^{2} \frac{4 p P}{(p+P)^{2}},
$$

where $\Lambda_{+}$is the positron thermal wavelength [Eq. (22)]. Above, $\xi$ is related to the trapping coefficient discussed in Sec. II.C.2, and it accounts for the overlap of the initial and final wave functions and for the energy release mechanism (electron-hole pairs). The last form in Eq. (59) assumes that $\xi$ is limited by the wave absorption (transmission) on the surface of the void. This term corresponds to the quantum reflection, which is discussed in Sec. II.C.6. In the case of small defects, $r_{v} \ll \Lambda_{+}, v$ becomes temperature independent, because $p \ll P$ for relevant initial positron momenta and depths of the defect potential. For large voids, $r_{v} \gg \Lambda_{+}, v$ becomes directly proportional to temperature, and, at zero temperature, it vanishes due to the quantum reflection. This argument is based on the reflection of the coming positron at the potential step. One can argue that, at the low initial positron momentum (very low temperatures), another inelastic channel becomes available for trapping. Phenomenologically, the trapping coefficient $v$ behaves at low temperatures as

$$
v=v_{0}+A T \text {. }
$$

$A$ as well as $v_{0}$ can be used as fitting parameters of the model. Actually, the term $v_{0}$ should vanish due to the quantum reflection, but the form of Eq. (60) with a finite $v_{0}$ corresponds better to the experimental situation (Nieminen et al., 1979).

The temperature dependence of the fraction $F$ of trapped positrons and the intensity $I_{2}$ can be discussed in terms of different regimes. At low temperatures, the positron mobility is high, i.e., the ratio

$$
\beta=\frac{4 \pi r_{v} D_{+}}{v} \gg 1 \text {. }
$$

In this case only the longest-living component in $f_{b}(r, t)$ contributes in the sum of Eq. (57), leading to this familiar expression for $I_{2}$ [cf. Eq. (35)],

$$
I_{2} \simeq \frac{v c_{v}^{\prime}}{v c_{v}^{\prime}+\lambda_{b}-\lambda_{t}}
$$

where $c_{v}^{\prime}=3 / 4 \pi\left(R_{v}^{3}-r_{v}^{3}\right)$ is an "effective" void density. The result is that at low temperatures $I_{2}$ rises like $v$, approximately linearly as a function of temperature. At high temperatures the positron mobility is low, $\beta \ll 1$, and one obtains

$$
I_{2} \simeq \frac{4 \pi c_{v}^{\prime} D_{+}}{4 \pi c_{v}^{\prime} D_{+}+\lambda_{b}-\lambda_{t}} .
$$

The intensity $I_{2}$ has become independent of the trapping coefficient $v$. It is determined through the depletion of the positron density at the void surface (see Fig. 17). When temperature rises, the intensity $I_{2}$ first saturates and finally, due to the temperature dependence of the diffusion coefficient, starts to decrease proportional to $T^{-1 / 2}$.

Trumpy and Bentzon (1992) suggest for positron trapping at voids a semiclassical model that is valid for all void sizes and temperatures. In the model, positrons can be trapped either directly to the ground state or via a "volume state" at the void. Using old quantum- 
mechanical calculations (Blatt and Weisskopf, 1952) as a base, they suggest that the positron-capture cross section can be approximated as

$$
\sigma=\pi\left(r_{v}^{2}+\Lambda_{+}^{2}\right) \xi
$$

instead of Eq. (59). This form is consistent with the linear temperature dependence of the trapping rate in Eq. (60), which they write in the form

$$
v=v_{0}(1+\alpha T) \text {. }
$$

Trumpy and Bentzon show that $\alpha$ depends on the void radius. It had previously been found (Bentzon and Evans, 1990) nearly independent of the void decoration by gas atoms or molecules.

A typical behavior of the temperature dependence of the trapping rate $\kappa$ is shown in Fig. 18. The figure compares the trapping rate measured by Bentzon and Evans (1990) for $N$-decorated voids in a Mo sample and a theoretical fit using the Nieminen et al. (1979) model. In the experimental work the mean void radius and density were determined by transmission electron microscope to be $13.3 \AA$ and $5.3 \times 10^{15} \mathrm{~cm}^{-3}$, respectively. The measured positron bulk and void lifetimes were $\tau_{b}=120 \mathrm{ps}$ and $\tau_{v}=630 \mathrm{ps}$, respectively. The experimental trapping rates result from a three-component lifetime spectrum analysis, with positron annihilation in shallow traps taken into account. The theoretical fit (made by us for this review) is calculated, however, within the two-stage trapping model, in which the trapping rate is related to the intensity $I_{2}$ via Eq. (35). The theoretical fit corresponds to the parameter values of $\mu_{0}=8.75 \times 10^{-7} \mathrm{~cm}^{3} / \mathrm{s}$, $A=6.5 \times 10^{-9} \mathrm{~cm}^{3} / \mathrm{sK}$, and $D_{+}(300 \mathrm{~K})=0.85 \mathrm{~cm}^{2} / \mathrm{s}$. The trapping parameters $\mu_{0}$ and $A$ are in good agreement with the simpler analysis made originally by Bentzon and Evans (1990). The positron diffusion coefficient is somewhat too low in comparison with its value of $D=1.1$ $\mathrm{cm}^{2} / \mathrm{s}$ determined from slow-positron-beam measure-

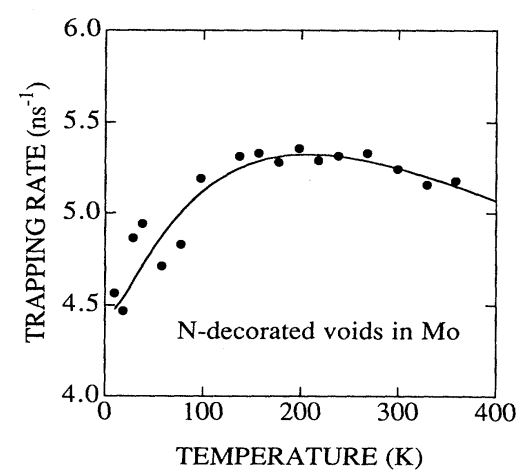

FIG. 18. Temperature dependence of positron trapping at voids decorated with nitrogen. The experimental points (Bentzon and Evans, 1990), denoted by solid circles, were obtained in a three-component analysis of positron lifetime spectra. The solid line is the theoretical fit using the model by Nieminen et al. (1979), Eq. (57), for $I_{2}$ and the two-stage trapping-model result (35) for $\kappa$. ments (Soininen et al., 1990). This may reflect effects due to shallow traps, which, for simplicity, are not included in our analysis. The computer code used in our analysis is available in a software server (SOFTWARE, 1993). The data shown in Fig. 18 were also recently analyzed by Trumpy and Bentzon (1992) using the model described above.

\section{Positron trapping at surfaces}

The image-induced potential well sensed by a positron at a solid surface can support bound states (see Sec. III.C). The trapping (sticking) of positrons into the surface state is an important issue in the slow-positron-beam experiments. Theoretically, the problem has been approached, for example, using Fermi-golden-rule calculations analogous to those for positron trapping into vacancies and voids. Neilson et al. (1986) calculated the trapping of thermalized positrons to the surface state using a model in which the electron states correspond to an infinite potential barrier at the surface, and in which the initial and final positron states are calculated approximating the potential by an asymmetric well at the surface. The energy lost is given to an electron-hole pair. The main interest of Neilson et al. (1986) was, however, the calculation of the energy losses of positrons reemitted from solids to vacuum.

Kong et al. (1990) studied the temperature dependence of positron trapping on $\mathrm{Ag}(111)$ and $\mathrm{Ag}(100)$ surfaces both experimentally and theoretically. The positrons are implanted in the solid and part of them diffuse back to the surface, where they can be trapped or emitted as free positrons or as positronium atoms. From the measured positronium yields Kong et al. (1990) concluded that the positron trapping rate is strongly enhanced for $\mathrm{Ag}(111)$ towards low temperatures, whereas for the $\operatorname{Ag}(100)$ it increases only moderately as temperature decreases. Kong et al. (1990) explained the result for Ag(111) using a model in which a positron is first trapped by a mirrorpotential-induced Rydberg state in a phonon-excitation process. The positron then makes the transition to the ground state via an electron-hole creation process. The phonon process leads to a trapping rate which increases strongly as the temperature decreases. This two-step model is analogous to that used for explaining positron trapping into negatively charged vacancies in semiconductors. According to Kong et al. (1990), the two-step model is possible for $\mathrm{Ag}(111)$, because its work function for positrons is negative. The work function for $\operatorname{Ag}(100)$ is positive, and Kong et al. (1990) explains that trapping via Rydberg states is not possible because the Rydberg states are higher in energy than the initial positron state in the bulk Ag. In this case only the direct trapping via an electron-hole process to the ground state is possible, and Kong et al. (1990) showed that this process results in a weak temperature dependence.

Recently, Walker et al. (1992) calculated the surface- 
state trapping rates using Fermi's golden rule. They extended the calculations by Neilson et al. (1986) to surface trapping. Walker et al. (1992) considered the trapping to occur either before or after positron implantation in the solid. They also modeled besides metal-vacuum surfaces, trapping into clean and He-filled voids. Walker et al. (1992) calculated the trapping rate as a function of the initial positron energy and showed that the Fermi-goldenrule formalism always leads to a vanishing trapping rate when temperature decreases towards absolute zero. This is because of the total reflection of the initial positron wave function when the positron kinetic energy vanishes. This result agrees with the measurements by Britton et al. (1989), but this kind of temperature dependence is not clear from the results of Kong et al. (1990). Moreover, Walker et al. (1992) found that surface resonances have no effect on the trapping process, but that the trapping rate is very sensitive to the positron work function, and, in the case of He bubbles, to the He density. Walker et al. (1992) concluded, on the basis of the agreement between experiments and their results, that the assumption of weak positron-surface coupling is valid. A sufficiently strong coupling (a positron trapped at the surface deforms the potential well) is argued to result in a sticking probability approaching unity as temperature decreases (Martin et al., 1991; Mills et al., 1991).

Finally, it is interesting to note that Brenig and Russ (1992) found that for the long-range Coulomb potential the sticking probability of a quantum-mechanical particle always has a nonzero limit when the energy of the incident particle approaches zero. In contrast, the sticking probability for a short-range potential (such as the van der Waals potential) vanishes in this limit. The latter theoretical result contradicts the experimental finding of Mills et al. (1991) that Ps atoms are not perfectly reflected from a surface at zero temperature.

\section{POSITRON STATES IN SOLIDS: MODELS}

\section{A. Two-component density-functional theory}

\section{Generalized Kohn-Sham method}

The foundation for modern electronic structure calculations for solids is the density-functional theory based on the work by Hohenberg and Kohn (1964) and by Kohn and Sham (1965). During the last decade the $a b$ initio electronic-structure-calculation methods developed rapidly, and nowadays most of the important basic properties of solids, such as the structural and cohesive properties, can be calculated without any adjustment to the experimental results (Jones and Gunnarsson, 1989). In this article, we are interested in how to calculate the positron states in solids and how to determine positron energy levels and annihilation characteristics, which are the experimental signals giving information about the electronic structure of the solid. It turns out that the $a b$ initio determination of positron states in solids is possible on the basis of the two-component generalization of the density-functional theory (Chakraborty and Siegel, 1983; Lundqvist and March, 1983; Nieminen et al., 1985; Boroński and Nieminen, 1986).

In two-component density-functional theory, the ground-state energy of a system of electrons and positrons in an external potential $V_{\text {ext }}$ is written as a functional of the electron $\left(n_{-}\right)$and positron $\left(n_{+}\right)$densities (Boroński and Nieminen, 1986),

$$
\begin{aligned}
E\left[n_{-},\right. & \left.n_{+}\right] \\
= & F\left[n_{-}\right]+F\left[n_{+}\right]+\int d \mathbf{r} V_{\mathrm{ext}}(\mathbf{r})\left[n_{-}(\mathbf{r})-n_{+}(\mathbf{r})\right] \\
& -\int d \mathbf{r} \int d \mathbf{r}^{\prime} \frac{n_{-}(\mathbf{r}) n_{+}\left(\mathbf{r}^{\prime}\right)}{\left|\mathbf{r}-\mathbf{r}^{\prime}\right|}+E_{c}^{e-p}\left[n_{+}, n_{-}\right],
\end{aligned}
$$

where $F[n]$ denotes the following one-component functional for electrons or positrons,

$$
F[n]=T[n]+\frac{1}{2} \int d \mathbf{r} \int d \mathbf{r}^{\prime} \frac{n(\mathbf{r}) n\left(\mathbf{r}^{\prime}\right)}{\left|\mathbf{r}-\mathbf{r}^{\prime}\right|}+E_{x c}[n] .
$$

$T[n]$ is the kinetic energy of noninteracting electrons or positrons, and $E_{x c}[n]$ is the exchange-correlation energy between indistinguishable particles. Finally, $E_{c}^{e-p}\left[n_{+}, n_{-}\right]$in Eq. (66) is the electron-positron correlation-energy functional.

The ground-state electron and positron densities minimizing $E\left[n_{-}, n_{+}\right]$can be calculated using a generalized Kohn-Sham method, which requires the solving of the following set of one-particle Schrödinger equations for electrons and positrons,

$$
\begin{aligned}
& -\frac{1}{2} \nabla^{2} \psi_{i}(\mathbf{r})+\left[\frac{\delta E_{x c}\left[n_{-}\right]}{\delta n_{-}(\mathbf{r})}-\phi(\mathbf{r})+\frac{\delta E_{c}^{e-p}\left[n_{+}, n_{-}\right]}{\delta n_{-}(\mathbf{r})}\right] \psi_{i}(\mathbf{r})=\epsilon_{i} \psi_{i}(\mathbf{r}), \\
& -\frac{1}{2} \nabla^{2} \psi_{i}^{+}(\mathbf{r})+\left[\frac{\delta E_{x c}\left[n_{+}\right]}{\delta n_{+}(\mathbf{r})}+\phi(\mathbf{r})+\frac{\delta E_{c}^{e-p}\left[n_{+}, n_{-}\right]}{\delta n_{+}(\mathbf{r})}\right] \psi_{i}^{+}(\mathbf{r})=\epsilon_{i}^{+} \psi_{i}^{+}(\mathbf{r}),
\end{aligned}
$$

where

$$
\phi(\mathbf{r})=\int d \mathbf{r}^{\prime} \frac{-n_{-}\left(\mathbf{r}^{\prime}\right)+n_{+}\left(\mathbf{r}^{\prime}\right)+n_{0}\left(\mathbf{r}^{\prime}\right)}{\left|\mathbf{r}-\mathbf{r}^{\prime}\right|}
$$

is the total Coulomb potential and $n_{0}(r)$ denotes the (positive) charge density providing the external potential $V_{\text {ext }}$. The electron and positron densities are calculated by summing over the occupied states $\left(\epsilon_{F}\right.$ is the electron 
Fermi energy and $N_{+}$is the number of positrons),

$$
n_{-}(\mathbf{r})=\sum_{\epsilon_{i} \leq \epsilon_{F}}\left|\psi_{i}(\mathbf{r})\right|^{2}, \quad n_{+}(\mathbf{r})=\sum_{i}^{N_{+}}\left|\psi_{i}^{+}(\mathbf{r})\right|^{2} .
$$

Equations (68)-(71) have to be solved self-consistently and simultaneously for electron and positron states, using an iterative scheme.

\section{Local-density approximation}

The above method [Eqs. (68)-(71)] would be exact if the exchange-correlation energy functional $E_{x c}[n]$ and the electron-positron correlation-energy functional $E_{c}^{e-p}\left[n_{+}, n_{-}\right]$were known. Unfortunately, this is not the case. In electronic structure calculations the most popular way to continue is to make the local-density approximation (LDA) for exchange-correlation effects. Local-density approximation means that the exchangecorrelation energy is approximated as

$$
E_{x c}[n]=\int n(\mathbf{r}) \epsilon_{x c}(n(\mathbf{r})) d \mathbf{r},
$$

where $\epsilon_{x c}(n)$ is the exchange-correlation energy per particle in a homogeneous one-component electron gas. In Eqs. (68) and (69), the functional derivatives of $E_{x c}[n]$ become a function of density. This function is called the exchange-correlation potential,

$$
\mu_{x c}(n)=\frac{\delta E_{x c}[n]}{\delta n(\mathbf{r})}=\frac{\partial\left[n \epsilon_{x c}(n)\right]}{\partial n} .
$$

The exchange-correlation energy $\epsilon_{x c}(n)$ for a homogeneous electron gas is well known from the quantum Monte Carlo simulations by Ceperley and Alder (1980). (Practical parametrizations of their local exchangecorrelation functional are given, for example, by Perdew and Zunger, 1981 and by Perdew and Wang, 1992.) The electron-positron correlation energy $E_{c}^{e-p}\left[n_{+}, n_{-}\right]$is best known in the case of the vanishing positron density in a homogeneous electron gas (see, for example, the works by Arponen and Pajanne, 1979a, 1979b, 1985). For finite positron densities, there exist results for some ratios of the positron and electron densities calculated by Lantto (1987). Using this data, Boronski and Nieminen (1986) studied the form of the electron-positron correlation energy and potential as a function of electron and positron densities. Requiring that the electron-positron correlation energy be symmetric with respect to the electron and positron densities, they ended up with interpolation formulas giving the energy function $E_{c}^{e-p}\left(n_{+}, n_{-}\right)$ defined per unit volume. This function can be used within LDA in practical calculations.

The positron annihilation rate is proportional to the overlap of positron and electron densities. In the two- component formalism within LDA the equation reads

$$
\lambda=\pi r_{0}^{2} c \int d \mathbf{r} n_{+}(\mathbf{r}) n_{-}(\mathbf{r}) g\left(0 ; n_{+}, n_{-}\right),
$$

where $g\left(0 ; n_{+}, n_{-}\right)$is the electron-positron paircorrelation function evaluated at the positron in a homogeneous two-component plasma with positron density $n_{+}$ and electron density $n_{-} \cdot r_{0}$ is the classical electron radius, and $c$ is the speed of light. The prefactor is the independent-particle model (IPM) or Sommerfeld result for the positron annihilation in a homogeneous electron gas with density $n_{-}$,

$$
\lambda_{0}=\pi r_{0}^{2} c n_{-} \text {. }
$$

Boroński and Nieminen (1986) presented a practical interpolation formula for the pair-correlation function $g\left(0 ; n_{+}, n_{-}\right)$, based on Lantto's (1987) many-body calculations. It has the correct high-density (i.e., the randomphase approximation) and low-density (i.e., the positronium atom) limits.

\section{Delocalized positron states}

For a delocalized positron in a perfect crystal lattice, the two-component density-functional theory simplifies essentially. Because the positron density in this case is vanishingly small at every point of the (infinite) lattice, it cannot influence the electronic structure. The electronic structure of the perfect lattice is therefore first solved by some standard self-consistent band-structure code. This code solves the Kohn-Sham equations obtained from Eqs. (68), (70), and (71) by omitting the positron density $n_{+}$ and the electron-positron correlation potential $\delta E_{c}^{e-p}\left[n_{+}, n_{-}\right] / \delta n_{-}(\mathbf{r})$. For example, the effective electron potential is

$$
v_{\text {eff }}(\mathbf{r})=-\phi(\mathbf{r})+v_{x c}\left(n_{-}(\mathbf{r})\right) .
$$

The potential sensed by the positron is constructed as the sum of the Coulomb potential $\phi$ (from the electronic structure calculation) and the correlation potential $V_{\text {corr }}$,

$$
V_{+}(\mathbf{r})=\phi(\mathbf{r})+V_{\text {corr }}\left(n_{-}(\mathbf{r})\right) .
$$

The correlation potential $V_{\text {corr }}$ is the zero-positrondensity limit of the electron-positron correlation potential $\delta E_{c}^{e-p}\left[n_{+}, n_{-}\right] / \delta n_{+}(\mathbf{r}) . V_{\text {corr }}$ is equal to the (correlation) energy for a delocalized positron in a homogeneous electron gas. A practical form for it is the parametrization by Boroński and Nieminen (1986), based on the data by Arponen and Pajanne (1979a, 1979b). For high electron density $r_{s} \leq 0.302\left(n_{-}=3 /\left(4 \pi r_{s}^{3}\right)\right)$, the RPA result is valid:

$$
V_{\text {corr }}\left(r_{s}\right)[\mathrm{Ry}]=-1.56 / \sqrt{r_{s}}+\left(0.051 \ln r_{s}-0.081\right) \ln r_{s}+1.14 .
$$

For $0.302 \leq r_{s} \leq 0.56$, 


$$
V_{\mathrm{corr}}\left(r_{s}\right)[\mathrm{Ry}]=-0.92305-\frac{0.05459}{r_{s}^{2}} ;
$$

for $0.56 \leq r_{s} \leq 8.0$,

$$
V_{\mathrm{corr}}\left(r_{s}\right)[\mathrm{Ry}]=-0.6298-\frac{13.15111}{\left(r_{s}+2.5\right)^{2}}+\frac{2.8655}{\left(r_{s}+2.5\right)} ;
$$

and for the low electron densities $r_{s} \geq 8.0$,

$$
V_{\text {corr }}\left(n_{-}\right)[\mathrm{Ry}]=-0.524-179856.2768 n_{-}^{2}+186.4207 n_{-} \text {. }
$$

This correlation energy is shown in Fig. 19 as a function of the electron-density parameter $r_{s}$. Over the density region $r_{s}=2, \ldots, 4$, which corresponds to typical electron densities in the interstitial regions of metal lattices, variations in the correlation energy are rather moderate, although positrons have a tendency to favor high-electrondensity regions as far as the correlation energy is concerned. At low electron densities the correlation energy approaches the total energy $-0.524 \mathrm{Ry}$ of the negative positronium ion $\mathrm{Ps}^{-}$.

When the potential sensed by a positron is obtained, the corresponding Schrödinger equation can be solved using the same band-structure code as that used for electrons. The solution of the positron wave function is needed only for a single delocalized positron in the system. Usually one is interested only in the positron wave function and the energy eigenvalue in the lowest energy state, i.e., at the bottom of the lowest positron energy band. This corresponds to the positron momentum $\mathbf{p}=0$, and the positron wave function for this state is $s$-type with respect to the nuclear positrons in the lattice.

When the positron and electron densities are known, the positron annihilation rate can be calculated according to Eq. (74). In the case of a positron delocalized in a perfect crystal lattice, one must use the zero-positrondensity limit of the pair-correlation function $g\left(0 ; n_{+}, n_{-}\right)$ at the positron site. This limit is denoted $\gamma\left(n_{-}\right)$and is called the enhancement factor of the electron density at the positron. Thus

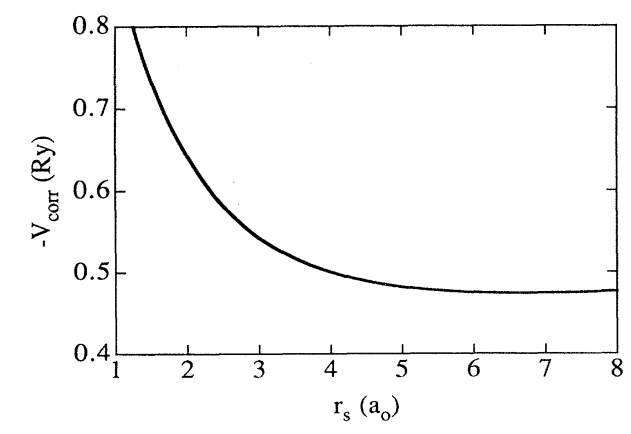

FIG. 19. Electron-positron correlation energy as a function of the electron-gas-density parameter (Arponen and Pajanne, 1979a, 1979b; Boroński and Nieminen, 1986). The correlation energy is the energy gained when a single positron is inserted into a homogeneous electron gas.

$$
\begin{aligned}
\lambda & =\pi r_{0}^{2} c \int d \mathbf{r} n_{+}(\mathbf{r}) n_{-}(\mathbf{r}) \gamma\left(n_{-}\right) \\
& =\int d \mathbf{r} n_{+}(\mathbf{r}) \Gamma\left(n_{-}(\mathbf{r})\right)
\end{aligned}
$$

where $\Gamma\left(n_{-}\right)$is the positron annihilation rate in a homogeneous electron gas with density $n_{-}$. Boroński and Nieminen (1986) give for $\Gamma\left(n_{-}\right)$the following interpolation formula based on the many-body calculations by Arponen and Pajanne (1979),

$$
\begin{gathered}
\Gamma(n)=\pi r_{0}^{2} c n\left(1+1.23 r_{s}+0.8295 r_{s}^{3 / 2}-1.26 r_{s}^{2}\right. \\
\left.+0.3286 r_{s}^{5 / 2}+\frac{1}{6} r_{s}^{3}\right) .
\end{gathered}
$$

The behavior of $\Gamma\left(n_{-}\right)$is shown in Fig. 20 as a function of the electron-density parameter $r_{s}$. The enhancement over the independent-particle results $\lambda_{0}$ [Eq. (75)] is substantial. For instance, the enhancement factor for a high-density electron gas corresponding to the density parameter $r_{s}=2$ is $\sim 4$ and increases rapidly to the value of $\sim 40$ when the electron density decreases to $r_{s}=6$. As a matter of fact, at low electron densities, the annihilation rate approaches the value of $2 \mathrm{~ns}^{-1}$, which corresponds to the annihilation rate of $\mathrm{Ps}^{-}$.

This picture for a delocalized positron in a perfect crystal lattice can be described another way. The electron density in the infinite lattice is not disturbed due to the positron, despite the short-range pileup (enhancement) at the positron site. Therefore the average electron density can be calculated first without any influences due to the positron. The positron state is calculated by tak-

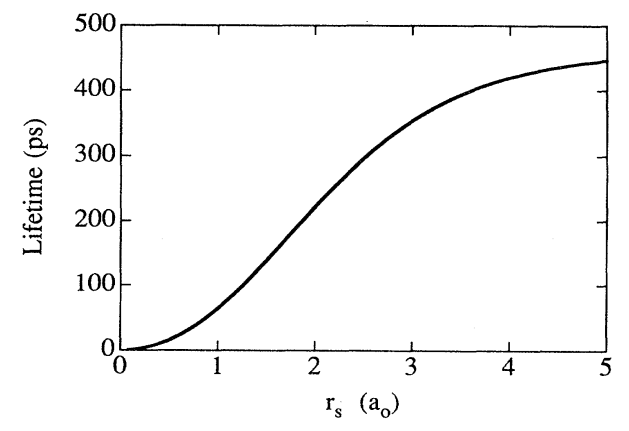

FIG. 20. Positron lifetime in a homogeneous electron gas as a function of the electron-gas-density parameter according to Boroński and Nieminen (1986). 
ing the electron pileup into account as a lowering of the energy (correlation energy), which in a given point depends on the electron density at that point only (LDA). Finally, the positron annihilation rate is calculated as an integral over the local enhanced annihilation rate weighted by the positron probability function.

\section{Localized positron states}

In the case of a positron localized at a lattice defect, the situation is more complicated because the positron attracts electrons, and the average electron density increases near the defect. In this case the electron and positron densities have to be solved simultaneously in a selfconsistent way. The short-range screening effects have to be taken into account by correlation functionals (correlation energy and pair-correlation function) which depend on both the electron and the positron densities. These functionals are somewhat poorly known, as there exist many-body calculations for only a small set of electron and positron densities. Moreover, the self-interaction effects for a localized positron inherent in the LDA twocomponent theory call for a careful treatment (see below). Thus far, in most applications for positron states at defects, the full scheme is simplified by using the same procedure as that for delocalized positrons. This means that the electron density is calculated without the positron influence, and the zero-positron-density correlation energy and enhancement factors are used. The simplified scheme can be justified by arguing that the positron, together with its screening cloud, forms a neutral quasiparticle that enters the system without changing the average electron density. This picture clearly breaks when the extent of the localized positron state decreases close to the extent of the short-range positron screening cloud. On the other hand, the two-component calculations performed (Nieminen et al., 1985; Boroński and Nieminen, 1986) support the use of the "conventional" scheme because the annihilation rates in these two methods are very similar. This similarity is due to the fact that the larger short-range enhancement compensates the smaller average electron density in the conventional scheme relative to the two-component calculation.

The two-component formalism just presented describes a single localized positron also as positive charge plasma. This raises the question, do the Coulomb self-interaction and exchange-correlation contributions for the positron plasma cancel each other properly? This cancellation requirement can be taken into account explicitly by making the self-interaction correction, i.e., by subtracting these contributions from the effective positron potential in Eq. (69). Hence

$$
\begin{aligned}
{\left[-\frac{1}{2} \nabla^{2}-\int\right.} & d \mathbf{r} \frac{n_{-}\left(\mathbf{r}^{\prime}\right)-n_{0}\left(\mathbf{r}^{\prime}\right)}{\left|\mathbf{r}-\mathbf{r}^{\prime}\right|} \\
+ & \left.\frac{\delta E_{c}^{e-p}\left[n_{+}, n_{-}\right]}{\delta n_{+}(\mathbf{r})}\right] \psi^{+}(\mathbf{r})=\epsilon^{+} \psi^{+}(\mathbf{r})
\end{aligned}
$$

is the self-interaction-corrected Schrödinger equation for the positron. See also Sec. IV.B.1.

\section{B. Positron states in semiconductors and insulators}

The above formulas for the electron-positron correlation potential and the electron enhancement work well only in the case of (simple) metals, because they are based on the calculations for a positron in a homogeneous electron gas. In semiconductors or insulators, the screening of the positron by electrons is not perfect due to the existence of the band gap. This must be taken into account in the electron-positron correlation energy when calculating the positron potential. It is also important to modify the expression for the electron contact density at the positron when calculating the annihilation rate. Puska, Mäkinen, et al. (1989) have presented two models that include reduction of the positron screening in semiconductors and insulators, respectively.

The model (Puska, Mäkinen, et al., 1989) for semiconductors is semiempirical and is based on the results for a positron in a homogeneous electron gas. In the model the annihilation rate [Eq. (82)] is replaced by

$$
\begin{gathered}
\Gamma\left(n_{-}\right)=\pi r_{0}^{2} c n\left[1+1.23 r_{s}+0.8295 r_{s}^{3 / 2}\right. \\
-1.26 r_{s}^{2}+0.3286 r_{s}^{5 / 2} \\
\left.+\frac{1}{6}\left(1-1 / \epsilon_{\infty}\right) r_{s}^{3}\right]
\end{gathered}
$$

where $\epsilon_{\infty}$ is the high-frequency dielectric constant. The form of this equation can be justified by two constraints for the screening cloud of the positron (Puska, Mäkinen, et al., 1989). Firstly, at the positron $(\mathbf{r}=0)$ the electron density $n(r)$ obeys the cusp condition

$$
\left.\frac{\partial n_{-}}{\partial r}\right|_{r=0}=-n(0) \text {. }
$$

Secondly, the screening cloud $\Delta n_{-}(r)$ induced by the positron has to contain $\left(1-1 / \epsilon_{\infty}\right)$ electrons, i.e.,

$$
\int_{0}^{\infty} \Delta n(r) 4 \pi r^{2} d r=\left(1-1 / \epsilon_{\infty}\right)
$$

This guarantees that the Coulomb potential due to the positron is the long-range potential proportional to $1 / \epsilon_{\infty} r$. The effects due to the reduced screening on the correlation potential are less pronounced, and they can be taken into account by noting that the correlation potential scales as $\sim\left(\lambda-\lambda_{0}\right)^{1 / 3}$ (Nieminen, 1983), where $\lambda$ is the actual annihilation rate and $\lambda_{0}$ is the annihilation rate when the enhancement is not taken into account. This semiempirical formulation has been shown to give for group-IV semiconductors as well as for III-V and II-IV compound semiconductors positron bulk lifetimes that are in good agreement with experiment (Puska, 1991a). This is remarkable because the LDA approach used does not contain any adjustable parameters (see Sec. IV.A.3).

In the case of insulators the screening efficiency of the 
valence electrons is reduced even more, due to the band gaps being larger, than those in the case of semiconductors. Therefore the starting point of the semiempirical formulation of Eq. (85), a positron in a free-electron gas, is no longer adequate. In their model for insulators Puska, Mäkinen, et al. (1989) correlate the electron enhancement with the (atomic) polarizability of the insulator, which in turn is related to the dielectric constant via the Clausius-Mossotti relation

$$
\alpha_{a t}=\frac{3 \Omega}{4 \pi} \frac{\epsilon_{\infty}-1}{\epsilon_{\infty}+2},
$$

where $\Omega$ is the unit-cell volume. Puska et al. assume that the enhancement factor depends directly on the polarizability and write

$$
\Gamma\left(n_{-}\right)=\pi r_{0}^{2} c n\left(1+A+B \Omega \frac{\epsilon_{\infty}-1}{\epsilon_{\infty}+2}\right) .
$$

$A=0.684$ and $B=0.0240 a_{0}^{3}$ are parameters, the values of which are obtained by fitting the calculated positron lifetimes of several insulators to the experimental ones. Thus the enhancement factor in this model is a constant depending on the insulator in question. However, Puska et al. show that both in open-structure semiconductors and in insulators the unit-cell volume $\Omega$ is, to a good accuracy, inversely proportional to the effective electron density seen by the positron.

\section{Positron states on solid surfaces}

When the positron resides inside the solid, the local approximation [Eq. (77)] for the positron correlation potential is reasonable. This is because the unperturbed electron density is quite isotropic around the positron in its typical interstitial positions, and therefore the screening cloud is also isotropic. Moreover, the screening cloud can be approximated in the same manner as that in a homogeneous electron gas with the electron density equal to that at the position. However, when the positron leaves the solid through the surface to the vacuum, the screening cloud is left behind in the solid. The situation becomes anisotropic and LDA breaks. Far from the surface the correlation potential approaches the classical image potential

$$
V_{\text {corr }}(z \rightarrow \infty) \rightarrow-\frac{\epsilon_{0}-1}{\epsilon_{0}+1} \frac{1}{4\left(z-z_{0}\right)},
$$

where $\epsilon_{0}$ is the static dielectric constant of the material, $z$ is the coordinate perpendicular to the surface, and $z_{0}$ defines the position of the image plane. The resulting total potential develops a well just outside the surface. This well may bind the positron in the direction perpendicular to the surface: the positron is said to be trapped in a surface state.

The problem in the actual calculations for positron states is the joining of the two regions: the bulk region where the LDA is valid and the region of the image potential. Nieminen and Puska (1983) used the superimposed-atom method (see Sec. III.D) in constructing the potential in the LDA and replaced this potential by the image potential outside the surface in the region where the image potential was higher. The image position $z_{0}$ was adjusted to give the experimental binding energy for the positron on one of the high-symmetry surfaces of the metal in question. For the perfect surfaces, a single value of $z_{0}$ was found sufficient, and the image potential did not show any corrugations. This model is called the smooth-mirror model. In order to study the possibility of positron trapping into vacancy-type defects on surfaces, the so-called corrugated-mirror model was found more realistic. In this model the mirror image potential shows the same corrugations as the electron density.

The calculation of the positron lifetime for the surface state must also be performed beyond the LDA. The method (Nieminen et al., 1984) consistent with the above correlation-potential description is to use the LDA for the annihilation rate in the solid and near the surface, where the LDA is also used for the correlation potential. In the region of the image potential, the positron is a bare particle, and therefore the annihilation rate is sharply reduced in this region.

Jensen and Walker (1988) have introduced a theory that goes beyond the LDA in the electron-positron correlation and thereby is able to join smoothly the LDA region inside the solid to the mirror image region far outside the surface. This theory is based on the weighted density approximation (WDA) by Gunnarsson et al. (1979). In the Jensen-Walker theory the total-energy functional [Eq. (66)] of the electron-positron system is used with the electron density $n_{-}^{0}(\mathbf{r})$ of the system calculated without taking into account the influence of the positron. The electron-positron correlation-energy functional is given as

$$
E_{c}^{e-p}\left[n_{+}, n_{-}\right]=-\int_{0}^{1} d \lambda \int d \mathbf{r} \int d \mathbf{r}^{\prime} \frac{n_{-}^{0}(\mathbf{r}) n_{+}\left(\mathbf{r}^{\prime}\right)\left[g_{d}\left(\mathbf{r}, \mathbf{r}^{\prime} ; n_{+}, n_{-}, \lambda\right)-1\right]}{\left|\mathbf{r}-\mathbf{r}^{\prime}\right|}
$$

where $g_{d}\left(\mathbf{r}, \mathbf{r}^{\prime} ; n_{+}, n_{-}, \lambda\right)$ is the displaced charge paircorrelation function and $\lambda$ scales the positron-electron interaction from the vanishing interaction $\lambda=0$ to the full, actual interaction $\lambda=1$. The screening cloud (induced electron density) around the positron at $\mathbf{r}^{\prime}$ is given by

$$
n_{c}\left(\mathbf{r}, \mathbf{r}^{\prime} ; \lambda\right)=n_{-}^{0}(\mathbf{r})\left[g_{d}\left(\mathbf{r}, \mathbf{r}^{\prime} ; n_{+}, n_{-}, \lambda\right)-1\right] .
$$

The overall neutrality of the system leads then to the sum rule

$$
\int_{0}^{1} d \lambda \int d \mathbf{r} n_{-}^{0}(\mathbf{r})\left[g_{d}\left(\mathbf{r}, \mathbf{r}^{\prime} ; n_{+}, n_{-}, \lambda\right)-1\right]=1 .
$$


In the WDA by Jensen and Walker (1988), the exact pair-correlation function $g_{d}\left(\mathbf{r}, \mathbf{r}^{\prime} ; n_{+}, n_{-}, \lambda\right)$ is replaced by $g_{d}^{h}\left(\left|\mathbf{r}-\mathbf{r}^{\prime}\right| ; \widetilde{n}_{-}, \lambda\right)$, which is the pair-correlation function for a homogeneous system in the zero-positrondensity limit. In practice, they use the scaled positronium approximation

$$
g_{d}^{h}\left(\left|\mathbf{r}-\mathbf{r}^{\prime}\right| ; \tilde{n}_{-}, \lambda\right)=\frac{\lambda^{3} \exp \left[-\lambda r / a\left(\tilde{n}_{-}\right)\right]}{8 \pi\left[a\left(\tilde{n}_{-}\right)\right]^{3} n_{-}}+1,
$$

where the scaling length $a\left(\widetilde{n}_{-}\right)$is given by the interpolation formula (Jensen and Walker, 1988)

$$
a\left(\tilde{n}_{-}\right)=\left(1+41.87 \tilde{n}_{-}\right)^{-1 / 3} .
$$

The potential sensed by the positron consists of the Coulomb and correlation parts [Eq. (77)]. The correlation potential is calculated from the correlation energy as a functional derivative $\delta E_{c}^{e-p}\left[\tilde{n}_{-}\right] / \delta n_{+}(\mathbf{r})$, where the electron density is obtained by requiring that the sum rule [Eq. (93)] be satisfied. The positron annihilation rate is calculated as in Eq. (74) using the pair-correlation function $g_{d}^{h}\left(\left|\mathbf{r}-\mathbf{r}^{\prime}\right|=0 ; n_{-}^{*}, \lambda=1\right)$, which is obtained from the sum rule (93) with $\lambda=1$, i.e., omitting the integration over $\lambda$.

Rubaszek $(1989,1991)$ has also made a WDA theory for positrons. Her theory differs from that by Jensen and Walker in two main points. First, Rubaszek uses in the pair-correlation function a screening length independent of the parameter $\lambda$ [in Eq. (94) the screening length is $\left.a\left(\tilde{n}_{-}\right) / \lambda\right]$. Secondly, she uses the sum rule

$$
\int d \mathbf{r} n_{-}^{0}(\mathbf{r})\left[g_{d}\left(\mathbf{r}, \mathbf{r}^{\prime} ; n_{+}, n_{-}, \lambda\right)-1\right]=\lambda
$$

instead of that of Eq. (93).

There is an alternative model for the positron-surface interaction. Platzman and Tzoar (1986) suggested that a positronium atom could be physisorbed on a metal surface. This model was constructed to explain the long positron lifetimes on surfaces and the insensitivity of the surface-state desorption energy to Ps to the changes of the electron work function. On the other hand, the model gives 2D-ACAR momentum distributions resembling those for free positronium, being thus too narrow compared to the measured distributions. Recently, the adsorption of positronium on metal surfaces was studied both experimentally (Mills et al., 1991) and theoretically (Martin et al., 1991) from the viewpoint of proving that the positronium sticking coefficient would not vanish when temperature was lowered towards absolute zero.

\section{Methods for positron states in solids}

In principle, all the standard methods of electronic structure calculations for bulk solids, such as the augmented plane wave (APW; Loucks, 1966), the KKR Green's function (Korringa, 1947; Kohn and Rostoker, 1947), and the linear muffin-tin orbital (LMTO; Andersen, 1975), can be adapted for positron calculations, in which one first solves for the self-consistent electron density, then calculates the positron wave function, and finally determines the positron annihilation rate. (There are difficulties with the pseudopotential methods because they do not treat the electron density inside the ion cores accurately.) The positron-defect interactions can be easily studied in the "conventional" scheme (omitting the full two-component calculation) by using either a Green'sfunction or a supercell method. The positron states at defects in solids can also be calculated using the supercell method. However, at least as far as the positron distribution and annihilation rates are considered, a non-selfconsistent electron density is often sufficient in the conventional scheme.

One of the more approximate methods is the so-called Stott's pseudopotential scheme (Kubica and Stott, 1974; Stott and Kubica, 1975). It employs the fact that the positron wave function is isotropic around the nuclei in the solid and that the behavior of the wave function over the whole crystal can be described by a slowly varying envelope function. One need therefore solve the spherical Schrödinger equation inside a Wigner-Seitz sphere for a potential which can be obtained from free-atom calculations. The envelope function is then obtained from a Schrödinger-like equation with a weak "pseudopotential." Moreover, in practice the envelope function is a plane wave corrected by a low-order perturbation theory. The details of Stott's pseudopotential scheme have been reviewed in several articles (see, e.g., Nieminen and Manninen, 1979 and Nieminen, 1983). The scheme has been used successfully, for example, to calculate such positron properties as ground-state wave functions, energies, and band masses in metals (Kubica and Stott, 1974; Stott and Kubica, 1975; Stott and West, 1978), in insulators (Nieminen, 1975), and in disordered alloys (Kubica et al., 1975). Moreover, it has been applied to positrons and Ps in fluids in the context of cluster or bubble formation, respectively (Nieminen et al., 1980; Rytsölä et al., 1984; Tuomisaari et al., 1986).

In the method developed by Puska and Nieminen (1983a), the electron density of the solid is approximated by the superposition of free-atom densities $n_{-}^{\text {at }}(r)$,

$$
n_{-}(\mathbf{r})=\sum_{\mathbf{R}} n_{-}^{\text {at }}(|\mathbf{r}-\mathbf{R}|),
$$

where $\mathbf{R}$ summation runs over the positions of the host nuclei. The potential sensed by the positron is constructed in a similar way,

$$
V_{+}(\mathbf{r})=\sum_{\mathbf{R}} V_{\text {Coul }}^{\text {at }}(|\mathbf{r}-\mathbf{R}|)+V_{\text {corr }}\left(n_{-}(\mathbf{r})\right)
$$

where $V_{\text {Coul }}^{\text {at }}$ is the Coulomb potential for a free atom. The electron density and the positron potential are calculated in the node points of a three-dimensional mesh, which forms the smallest polyhedron capable by symmetry to describe the potential and wave functions in the whole lattice. The Schrödinger equation is then solved by a numerical relaxation technique (Kimball and Short- 
ley, 1934; Puska and Nieminen, 1984) for the positron eigenenergy and for the positron wave function at the node points. The relaxation technique is based on the discretization of the Schrödinger equation. In particular, the Laplace operator $\nabla^{2}$ at a given point is written using the values of the function at the six neighboring points. The positron wave function and the energy eigenvalue are determined in an iterative process. For example, in a one-dimensional case the equations to be solved are as follows.

(a) During the $n$th iteration cycle, the energy eigenvalue $\epsilon^{(n)}$ corresponding to the wave function $\psi^{(n)}$ determined at the mesh points indexed by $j$ is calculated as

$\epsilon^{(n)}=\frac{\sum_{j}\left[-\frac{1}{2 h^{2}} \psi_{j}^{(n)}\left[\psi_{j+1}^{(n)}-2 \psi_{j}^{(n)}+\psi_{j-1}^{(n)}\right]+V_{j}\left|\psi_{j}^{(n)}\right|^{2}\right]}{\sum_{j}\left|\psi_{j}^{(n)}\right|^{2}}$,

where $h$ is the constant distance between the mesh points.

(b) The wave function is then improved by the following step derived directly from the discretized Schrödinger equation,

$$
\psi_{i}^{(n+1)}=\frac{\left[\psi_{i+1}^{(n)}+\psi_{i-1}^{(n)}\right]}{2+2 h^{2}\left(V_{i}-\epsilon^{(n)}\right)} .
$$

Delocalized states are obtained by using boundary conditions, which continue the wave function through the polyhedron surfaces. Normally, only the $\mathbf{k}=0$ state is needed; but by using proper boundary conditions, it is possible to calculate states corresponding to nonzero $\mathbf{k}$ vectors. For localized states, the wave function is required to vanish on the polyhedron surface far enough from the center of the trap site. It is also possible to obtain (excited) states belonging to different symmetry representations (Puska and Nieminen, 1984). The binding energy at a defect is obtained as the difference between the delocalized- and localized-state energy eigenvalues. The method converges usually "safely" towards the lowest energy state consistent with the boundary conditions imposed for the wave function. The convergence may, however, be rather slow, especially if the number of the node points is large.

When the positron wave function and the electron density are known, the annihilation rate can be calculated according to Eqs. (82), (83), and (85) by a numerical integration in the three-dimensional mesh. In the original work by Puska and Nieminen (1983a) and in many successive works, the total annihilation rate was calculated as a sum of the contributions with valence, core, and possibly with the outermost $d$ electrons. Different enhancement factors were used with the different contributions, and the enhancement factor for $d$ electrons was even used as an adjustable parameter. Jensen (1989) realized that this division was not necessary, but that one could use the total electron density in calculating the enhancement and the total annihilation rate. Thus the electron density appearing in Eqs. (83) and (85) has to be understood as the total electron density. This LDA approach for the total annihilation rate is related to the local-density approximation for calculating the momentum densities of the annihilating positron-electron pairs (Daniuk et al., 1987; Jarlborg and Singh, 1987). The former LDA can be obtained from the latter by integrating over all the momenta. The LDA formulation using the total electron density as the only input is in accordance with the density-functional theory, which states that the total density is the physical quantity and, in principle, a division to different density contributions is not justified. Moreover, this LDA formulation is a pure ab initio method with no adjustable parameters.

The superimposed-atom method by Puska and Nieminen (1983a) is fast compared to the methods that determine the self-consistent electron density first. This is true especially for defects in solids. The superimposed-atom method can handle defects with low symmetry, which is important for cases in which the influence of the actual defect geometry determines to a large extent the positron annihilation characteristics.

The comparisons (Puska et al., 1986; Puska, 1991a) between the results obtained by using superimposed atom method and by using self-consistent electronic structures have shown that the positron annihilation rate is rather insensitive to the details of the self-consistency of the electronic structure. This is because the positron lifetime depends on the integral over the product of positron and electron densities. The positron density relaxes following electron charge transfer, tending to conserve the value of the overlap integral. These effects are clearly demonstrated in Fig. 21 for the perfect MgO lattice (Puska, 1992), in which there is a strong charge transfer from $\mathrm{Mg}$ atoms to $\mathrm{O}$ atoms. The self-consistent electron density and the positron states are calculated with the linear-

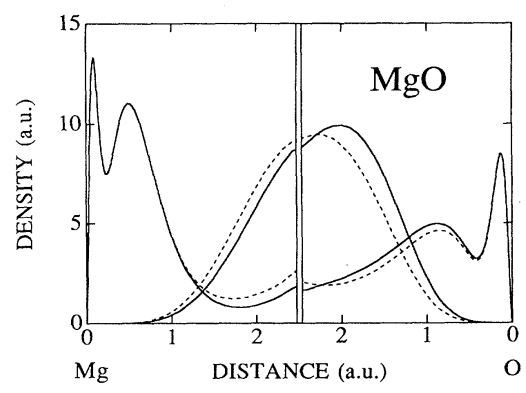

FIG. 21. Radial electron density $\left[4 \pi r^{2} n_{-}(r)\right]$ and positron density $\left[4 \pi r^{2}\left|\psi^{+}(r)\right|^{2}\right]$ around the $\mathrm{Mg}$ and $\mathrm{O}$ nuclei in $\mathrm{MgO}$. The positron density has been arbitrarily scaled for the figure. The self-consistent electron density as well as the positron densities were obtained using the LMTO-ASA method. The nonself-consistent electron density was obtained by superimposing free-atom densities and averaging spherically the threedimensional density around the nuclei. The densities corresponding to self-consistent and non-self-consistent calculations are denoted by solid and dashed curves, respectively (from Puska, 1992). 
muffin-tin-orbital method (LMTO) within the atomicspheres approximation (ASA) (Andersen, 1975; Skriver, 1984; Andersen et al., 1985, 1987). First a non-selfconsistent electron density (dashed line) is constructed by superposing free $\mathrm{Mg}$ and $\mathrm{O}$ atoms and calculating the spherical averages around the nuclei. The radii of the space-filling (and therefore slightly overlapping) $\mathrm{Mg}$ and $\mathrm{O}$ spheres are equal. Figure 22 shows the radial electron densities $\left[4 \pi r^{2} n_{-}(r)\right]$. The total charges (electrons and nuclei) inside the $\mathrm{Mg}$ and $\mathrm{O}$ spheres are about +0.5 and -0.5 electrons, respectively. When the electron density is relaxed to the self-consistent one (solid line), electron charge is shifted from the $\mathrm{Mg}$ sphere to the $\mathrm{O}$ sphere; so the final total charges of the $\mathrm{Mg}$ and $\mathrm{O}$ spheres are about +1.0 and -1.0 electrons. The positron potentials corresponding to the electron densities are calculated within the LDA [Eq. (77)]. For the non-self-consistent electron density, there are a total of 0.476 and 0.523 positrons inside the $\mathrm{Mg}$ and $\mathrm{O}$ spheres, respectively, whereas for the self-consistent electron density the corresponding numbers are 0.454 and 0.546 . The nearly equal distribution of the positron density between the $\mathrm{Mg}$ and $\mathrm{O}$ spheres is due to the fact that the positron density, especially the
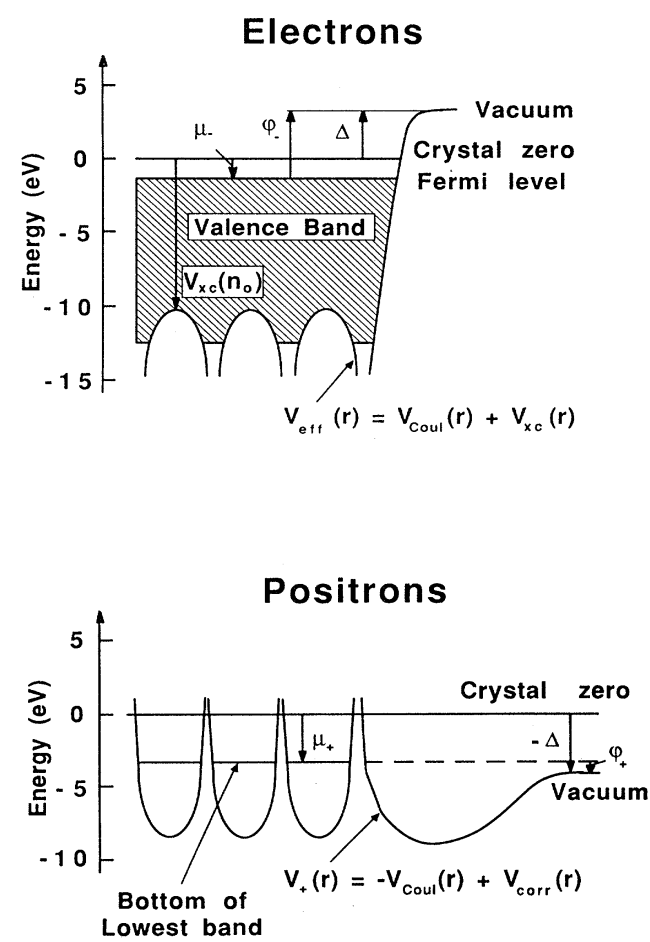

FIG. 22. Electron and positron potentials and energy levels near a metal surface. The vertical scale corresponds to the case of Al. $\Delta$ is the dipole potential at the surface, $v_{x c}\left(n_{0}\right)$ is the exchange-correlation potential in the interstitial region, and $\phi_{-}$ and $\phi_{+}$denote the electron and positron work functions, respectively. $\mu_{-}$and $\mu_{+}$are the electron and positron chemical potentials, respectively. Arrows pointing upwards and downwards denote positive and negative quantities, respectively (from Puska, Lanki, and Nieminen, 1989). radial density, is peaked around the interstitial regions. Because the positron density follows the electron density, the positron lifetimes calculated within the semiconductor model of screening for the non-self-consistent and self-consistent electron densities are nearly equal, i.e., 123 and $122 \mathrm{ps,}$ respectively. The superimposed atom method with the full three-dimensional treatment of the non-self-consistent electron density and positron wave function gives a somewhat longer lifetime of $130 \mathrm{ps.} \mathrm{This}$ reflects the difficulty of the ASA (with equal sphere radii) in describing the sodium chloride structure. For fcc and bcc metals, as well as for the diamond and zinc-blende structure semiconductors (which are treated in ASA with the so-called empty, interstitial spheres), the differences in the positron lifetimes calculated by the LMTO-ASA and superimposed atom method are of the order of $1 \mathrm{ps.}$

The positron energetics, on the other hand, is much more sensitive to the self-consistency of the electronic structure than the positron lifetime. For example, in the atomic superposition method, the positron energy eigenvalue in a perfect bulk should be the negative of the positron work function. This result is related to the notion that, in a similar construction of the electron potential, the so-called Mattheiss construct (Mattheiss, 1964), the electron work function is directly the negative of the Fermi energy of the electron bands (Weinert and Watson, 1984). However, the superimposed-atom calculations (Puska and Nieminen, 1983a) give eigenenergies that are much too high (too negative positron work functions). This means that the superimposed electron density at the surface spills too much to the vacuum. One way to improve the energetics in this case is through the use of contracted atomic densities. These have been used in calculating electron work functions (Weinert and Watson, 1984) and total energies of solids (Finnis, 1990; Polatoglou and Methfessel, 1990) by using superimposed atomic densities. This trick indeed improves the positron work functions from the superimposed-atom calculations (Puska, 1992).

In addition to the above method for calculating positron states, the superposition of free-atom densities has been used in various solid-state problems. Mattheiss (1964) constructed crystal potentials for use in bandstructure calculations. The Mattheiss construction has commonly been used for the starting potentials in selfconsistent electronic structure calculations. The superposition idea has been used as the basis for approximate methods for calculating the total energies of systems of interacting particles. For example, it has been used in the so-called effective-medium theory (Nørskov, 1982; Jacobsen et al., 1987), in which the energetics is calculated by using the immersion energies of free atoms into a homogeneous electron gas (Puska et al., 1981; Stott and Zaremba, 1982). In the recent method by Harris (1985) and Foulkes and Haydock (1989), the superimposed electron density and the corresponding effective potential are used in the calculation of the total energy of the manyatom system [small molecules (Harris, 1985; Foulkes and 
Haydock, 1989), solids (Methfessel et al., 1989; Polatoglou and Methfessel, 1990), surfaces and defects in solids (Finnis, 1990)]. The viability of the superimposed-atom method was recently systematically studied in the case of the total-energy calculations (Chetty et al., 1991; Robertson et al., 1991).

The use of the superposition of the spherical atomic densities might at first sound unsuitable for covalent semiconductors which have directed bonds. However, both the approximate total-energy calculations (Polatoglou and Methfessel, 1990) and the positron lifetime calculation with the method of Puska and Nieminen (1983a) give very good results in the comparison with selfconsistent methods. In the case of positron-state calculations, this means that the atomic superposition is able to describe well, in an average sense, both the electron density in the interstitial region and the shape of the repulsive potential near the ion core, thus giving the extent of the effective open volume available for the positron.

The relaxation method for solving the positron wave function is not restricted to use with atomic superposition. The electron density and the Coulomb potential can be determined on a point mesh using self-consistent electronic structure calculations. Because the positron wave function is relatively smooth and resides mainly in the open regions of the lattice, an already quite sparse mesh gives accurate results for the positron energy and annihilation rate. This kind of approach has been used, for example, with the LCAO (linear combination of atomic orbitals) band-structure method (Bharathi et al., 1990) and the pseudopotential plane-wave approach (Alatalo et al., 1993).

\section{E. The momentum distribution of electron-positron pairs}

In addition to the two-component density-functional theory (and its approximation for localized states), a different picture can be used to describe the positron interaction with its electronic environment in a solid (see, e.g., Daniuk et al., 1991). This picture may provide a more elegant basis for the calculation of the momentum distribution of the annihilating electron-positron pairs than the two-component density-functional theory. The reason is that, in these calculations, the single-electron wave functions are needed; in density-functional theory, in principle only the total densities, not the wave functions, are the physical quantities. On the other hand, it may be difficult to find "true" one-particle wave functions for electrons in solids; the only practical electronic structure calculations employ density-functional theory. The calculated momentum distributions are compared with the measurements of angular correlation of annihilation radiation (ACAR).

In the alternative approach the positron wave function is solved for the potential, which consists only of the Coulomb potential due to the nuclei and the electron density, which is not perturbed by the positron. The positron-electron correlation effects are taken into ac- count only when calculating the annihilation characteristics, the momentum distribution of the annihilating electron-positron pairs and its integral, and the total annihilation rate. Thus one writes for the momentum distribution

$$
\rho(\mathbf{P})=\sum_{i}\left|\int d \mathbf{r} e^{-i \mathbf{P} \cdot \mathbf{r}} \psi_{i}^{e p}(\mathbf{r}, \mathbf{r})\right|^{2},
$$

where $\mathbf{P}$ is the total momentum of the annihilating pair. $\psi_{i}^{e p}\left(\mathbf{r}, \mathbf{r}^{\prime}\right)$ is the two-particle wave function approximated as

$$
\psi_{i}^{e p}\left(\mathbf{r}, \mathbf{r}^{\prime}\right)=\psi_{+}^{0}(\mathbf{r}) \psi_{i}^{0}\left(\mathbf{r}^{\prime}\right) \sqrt{g_{i}(\mathbf{r})},
$$

where $\psi_{+}^{0}(\mathbf{r})$ and $\psi_{i}^{0}\left(\mathbf{r}^{\prime}\right)$ are the positron and electron wave functions calculated without positron-electron correlation effects. The $i$ summation in Eq. (101) is over all occupied electron states. The correlations are included via the function $g_{i}(\mathbf{r})$, which describes the distortion of the positron wave function from $\psi_{+}^{0}(\mathbf{r})$ as well as the enhancement of the electron density at the positron for the electron state $i$. The total annihilation rate can be obtained by integrating over all momenta,

$$
\begin{aligned}
\lambda & =\frac{\pi r_{0}^{2} c}{(2 \pi)^{3}} \int d \mathbf{P} \rho(\mathbf{P}) \\
& =\pi r_{0}^{2} c \int d \mathbf{r} \sum_{i}\left|\int d \mathbf{r} \psi_{i}^{e p}(\mathbf{r}, \mathbf{r})\right|^{2} .
\end{aligned}
$$

The correlation function $g_{i}(\mathbf{r})$ in Eq. (102) should be calculated using many-body techniques. The simplest approximation is the IPM, in which correlations are omitted altogether: $g_{i}(\mathbf{r})=1$. As a matter of fact, the IPM gives a rather good description for the momentum distribution $\rho(\mathbf{P})$ for valence electrons in simple metals. This is because the momentum dependence of $\rho(\mathbf{P})$ due to electron-positron correlation is not very strong for the valence electrons (Kahana, 1960, 1963). The electronpositron correlations cancel, in part, the effects due to electron-electron interactions. A simple step beyond the IPM is to use the momentum-dependent enhancement function $\epsilon\left(P, r_{s}\right)$ (Kahana, 1960, 1963) for the homogeneous electron gas as the correlation function (Mijnarends and Singru, 1979)

$$
g_{\mathrm{k} i}(\mathbf{r})=\epsilon\left(\sqrt{\epsilon_{\mathrm{k} i} / \epsilon_{F}} k_{F}, r_{s}\right),
$$

where $\epsilon_{\mathrm{k} i}$ is the energy of the electron in state $i$ and momentum $\mathbf{k}$, and $\epsilon_{F}$ is the Fermi energy. Both of these energies are measured from the bottom of the conduction band. Above, $r_{s}$ corresponds to the average electron density of the solid, and thus there is no $r$ dependence in the correlation function. The $\mathbf{r}$ dependence can be reintroduced by using a position-dependent $r_{s}$ parameter (Daniuk et al., 1987; Jarlborg and Singh, 1987). One then obtains the following equation in LDA,

$$
g_{\mathbf{k} i}(\mathbf{r})=\epsilon\left(\sqrt{\epsilon_{\mathbf{k} i} / \epsilon_{F}} k_{F}, r_{s}(\mathbf{r})\right),
$$

where $r_{s}(\mathbf{r})=\left[4 \pi n_{-}(\mathbf{r}) / 3\right]^{-1 / 3}$ depends on the electron 
density at point $\mathbf{r}$. This LDA correlation function has also been used in the case of core states that lie below the bottom of the conduction band by substituting zero for the electron momentum (Daniuk et al., 1989, 1991). Thus $g_{\mathbf{k} i}(\mathbf{r})=\epsilon\left(0, r_{s}(\mathbf{r})\right)$ for core states.

Taking the periodicity of the perfect crystal lattice into account, the momentum distribution of Eq. (101) can be written as

$$
\begin{aligned}
& \rho(\mathbf{P})=2 \sum_{n, \mathbf{k}} f(n, \mathbf{k})\left|A_{n}(k, \mathbf{p})\right|^{2} \\
& A_{n}(\mathbf{k}, \mathbf{p})= \delta(\mathbf{P}-\mathbf{k}-\mathbf{K}) \\
& \times \int_{\Omega} d \mathbf{r} e^{-i \mathbf{P} \cdot \mathbf{r}} \psi_{+}^{0}(\mathbf{r}) \psi_{n, \mathbf{k}}^{0}(\mathbf{r}) \sqrt{g_{n, \mathbf{k}}(\mathbf{r})},
\end{aligned}
$$

where $f(n, \mathbf{k})$ is the Fermi function (the factor 2 is due to the spin degeneracy), $\psi_{n, \mathbf{k}}^{0}(\mathbf{r})$ is the electron Bloch state with band index $n$ and wave vector $\mathbf{k}, \Omega$ is the volume of the primitive unit cell, and $\mathbf{K}$ denotes a reciprocal-lattice vector such that $\mathbf{P}-\mathbf{k}$ lies in the first Brillouin zone. The parts of the ACAR spectra, for which $K$ is nonzero, are called umklapp components. The practical importance of the umklapp components lies, for example, in the notion (Haghigli et al., 1991a, 1991b) that Fermi breaks in Eq. (106) may be seen more clearly in the region of a certain umklapp component than near the central peak corresponding to $\mathbf{K}=0$. Moreover, calculations show that the umklapp components of the ACAR spectra are more sensitive to the approximations of the theory than the $\mathbf{K}=0$ component, and therefore they are good test material for different theoretical approaches. For example, Sorman and Šob (1990) have studied the effects due to the approximations in the positron potential. Sorman (1991) has demonstrated the sensitivity of the positron wave function to the anisotropy. Sorman and Šob (1990) have also shown that the enhancement for the umklapp components may be smaller than for the $K=0$ component. Moreover, Singh and Jarlborg (1985) have developed a method to correct effectively the effects of the ASA in the LMTO calculations for the umklapp components.

The one- or two-dimensional ACAR curves, relevant for comparison with experiments, are related to the three-dimensional momentum distribution through partial integrations. The one-dimensional ACAR curve is obtained as

$$
\rho\left(\Theta_{z}\right) \sim \int d P_{x} \int d P_{y} \rho\left(P_{x}, P_{y}, \Theta_{z} m_{e} c\right),
$$

where $\Theta_{z}$ corresponds to the deviation of the angle between two annihilation gamma from $180^{\circ}$. For the freeelectron gas, for which the density of states is constant in the momentum space and the electron states are occupied up to the Fermi level, the one-dimensional ACAR curve in the IPM is an inverted parabola,

$$
\rho\left(\Theta_{z}\right) \sim\left[k_{F}^{2}-\left(\Theta_{z} m_{e} c\right)^{2}\right] .
$$

In addition to the conduction-electron contribution, which is described reasonably well by the above parabola in the case of simple metals, there is a broad Gaussiantype contribution in the ACAR curves. This contribution results from the annihilation with core electrons and from higher momentum (umklapp) components of the conduction electrons. The core and umklapp components can be partially separated using the Lock, Crisp, and West construction (Lock et al., 1973; Lock and West, 1975), but their unambiguous determination is, in practice, difficult.

There are two earlier approximations in which the momentum distribution was determined directly from the electron density so that individual electron wave functions were not needed. Brandt (1967) suggested that the one-dimensional ACAR curve be calculated in a local approximation in which a free-electron parabola corresponds to the electron density at every point,

$\rho\left(\Theta_{z}\right) \sim \int d \mathbf{r}\left|\psi_{+}(\mathbf{r})\right|^{2} \lambda\left[n_{-}(\mathbf{r})\right]\left[k_{F}(\mathbf{r})^{2}-\left(\Theta_{z} m_{e} c\right)^{2}\right]$.

In the so-called mixed-density approximation (Arponen et al., 1973), the nonlocal character of the momentum distribution is partly taken into account by writing for the partial annihilation rate at momentum $\mathbf{P}$

$$
\lambda(\mathbf{P}) \sim \int d \mathbf{r} \int d \mathbf{r}^{\prime} e^{i \mathbf{P} \cdot\left(\mathbf{r}-\mathbf{r}^{\prime}\right)} \psi_{+}^{*}(\mathbf{r}) \psi_{+}\left(\mathbf{r}^{\prime}\right) g\left\{k_{F}\left[\left(\mathbf{r}-\mathbf{r}^{\prime}\right) / 2\right]\left|\mathbf{r}-\mathbf{r}^{\prime}\right|\right\} \sqrt{\lambda\left[n_{-}(\mathbf{r})\right] \lambda\left[n_{-}\left(\mathbf{r}^{\prime}\right)\right]},
$$

where $g$ is related to the electron-electron paircorrelation function

$$
g(z)=\frac{3}{z^{3}}[\sin (z)-z \cos (z)]
$$

The mixed-density approximation has recently been used in the superimposed-atom method, and ACAR curves for positrons trapped at Al surfaces have been calculated (Brown et al., 1988).

\section{POSITRON STATES IN SOLIDS: RESULTS AND DISCUSSION}

\section{A. Delocalized positron states in perfect solids}

As discussed earlier, the two-component densityfunctional theory for electron and positron densities in solids simplifies considerably if the positron wave function is delocalized over the whole crystal lattice. Calculations obeying exactly the principles of the two- 
component theory are therefore easy to perform with some standard electron band-structure codes. In the case of metals, one obtains for the positron properties firstprinciples results, which are affected only by the LDA exchange and correlation functionals calculated for a positron in a homogeneous electron gas. In the case of semiconductors, one can use the model by Puska, Mäkinen, et al. (1989), but the results are semiempirical in the sense that the screening model relies on the measured dielectric constants of the solids. In Secs. IV.A.1 and IV.A.2, we shall discuss the first-principles results for the positron energetics and thereafter, in Sec. IV.A.3, those for the annihilation rates in perfect solids. Comparisons with experiments serve as good tests for the approximation made, especially for the main approximation, LDA.

\section{Positron and electron energy levels in solids}

The positron energetics in perfect solids can be characterized by two measurable quantities, the positron work function and the positronium formation potential (see, e.g., Schultz and Lynn, 1988). A useful theoretical concept which arises in this context is the positron affinity. The positron work function depends on the properties of the surface, whereas the positronium formation potential is a pure bulk property. A related quantity is the positron deformation potential, which was encountered in Sec. II.B dealing with positron diffusion in solids. The relations of the work functions and the positronium formation potential with the more theoretical concepts of the electron and positron energetics are discussed in the next paragraphs, after which results of actual calculations are reviewed. The positron deformation potential is dealt with in Sec. IV.A.2.

It is important in the discussion of absolute electron and positron energy levels that a common reference level be uniquely defined for both electrons and positrons. The band-structure calculations refer to perfect infinite lattices without a surface. Therefore the energy levels in the calculations are measured relative to an internal quantity, the so-called crystal zero. For instance, the electron $\left(\mu_{-}\right)$chemical potential is defined (see Fig. 22) as the distance of the Fermi level from the crystal zero. Similarly, the distance of the lowest positron energy level $\left(\mu_{+}\right)$from the crystal zero is the positron chemical potential $\left(\mu_{+}\right)$. The crystal zero is related to the Coulomb potential and it can be defined, for example, as the average electrostatic potential in the infinite lattice, or the average value of the electrostatic potential on the surface of the Wigner-Seitz cell. In the atomic spheres approximation (ASA), the results of which are reviewed below, the crystal zero is the Coulomb potential far away from a single atomic sphere (for neutral spheres, which are used, for example, for fcc and bcc metals, the Coulomb potential vanishes at their boundaries).

When the solid is considered to have a surface facing the vacuum, the distance from the electrostatic potential level, which a test charge feels in the vacuum (the vacu- um level), to the crystal zero is the surface dipole $\Delta$ (see Fig. 22). An electron and a positron feel the same surface dipole, but the effects to the energy positions are opposite due to the opposite positron and electron charges. The surface dipole depends on the electronic structure of the surface, especially on the spilling of the electron density into the vacuum. It also depends on the presence of adsorbates. The electron work function $\phi_{-}$is the distance between the absolute Fermi level and the vacuum level.

In the case of positrons, one need solve only the lowest energy state. The position of this energy level relative to vacuum defines the positron work function $\phi_{+}$. The magnitudes of the surface dipole and the chemical potentials are linked to the definition of the crystal zero. However, the electron and positron work functions are calculated as $\phi_{-}=\Delta-\mu_{-}$and $\phi_{+}=-\Delta-\mu_{+}$and do not depend on the position of the crystal zero.

Figure 23 shows the electron and positron energy levels in the case of two metals in contact. The Fermi levels equalize themselves over the interface as a result of the formation of an interface dipole with a potential difference of $\Delta=\mu_{-}^{A}-\mu_{-}^{B}$. The positron feels this potential; therefore the difference between the lowest positron energies on the different sides of the interface is given by

$$
\Delta E_{+}^{A, B}=E_{+}^{A}-E_{+}^{B}=\mu_{-}^{A}-\mu_{-}^{B}+\mu_{+}^{A}-\mu_{+}^{B} .
$$

This equation describing the behavior of the positron energy level in different regions of an inhomogeneous
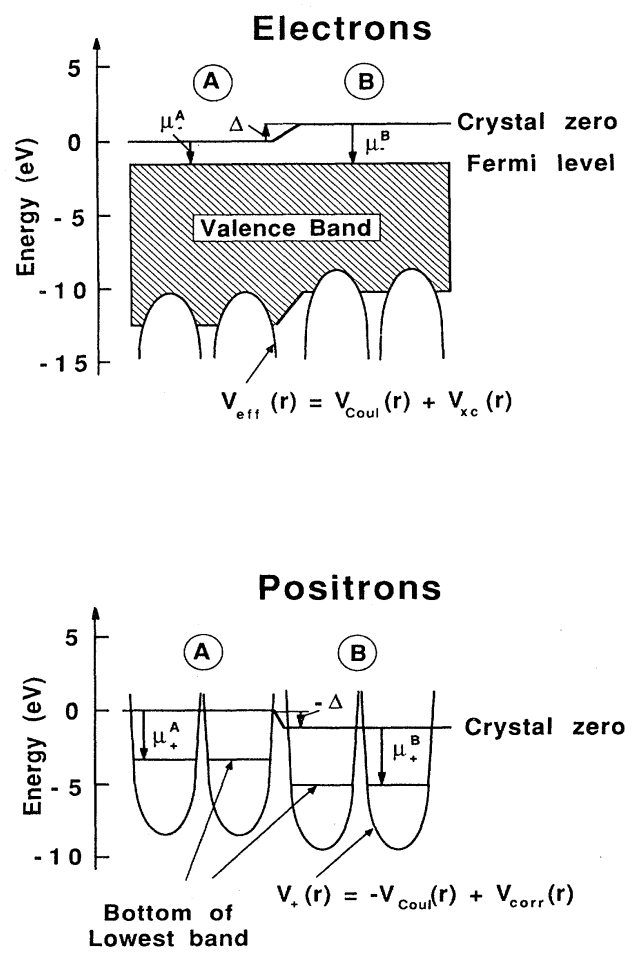

FIG. 23. Electron and positron potentials and energy levels near a junction of two metals. The vertical scale corresponds to an Al-Zn junction. See also the caption of Fig. 22 (from Puska, Lanki, and Nieminen, 1989). 
solid shows that it is useful to define the following quantity, which is a bulk property of a given homogeneous material (not related to the interface between two materials),

$$
A_{+}=\mu_{-}+\mu_{+} \text {. }
$$

This sum is called the positron affinity. The sign convention here is such that the more negative the positron affinity is, the deeper will be the positron energy level in the solid. According to this definition, the difference [Eq. (113)] of the positron energies between two materials in contact is

$$
\Delta E_{+}^{A, B}=A_{+}^{A}-A_{+}^{B}
$$

The positronium formation potential $\phi_{\mathrm{Ps}}$ is the negative of the maximum kinetic energy of Ps atoms ejected from the solid into the vacuum (see, e.g., Schultz and Lynn, 1988). The positronium formation potential is related to the positron affinity. Ps is not stable in bulk metals or semiconductors, but is formed near the surface when a delocalized positron leaves the solid. The extraction of a thermalized positron and a Fermi-level electron costs the sum of the two work functions. When forming Ps outside the surface, the binding energy of $E_{\mathrm{Ps}}=6.8 \mathrm{eV}$ is gained. The energy balance gives for the Ps formation potential

$$
\begin{aligned}
\epsilon_{\mathrm{Ps}} & =\phi_{-}+\phi_{+}-E_{\mathrm{Ps}} \\
& =-A_{+}-E_{\mathrm{Ps}} .
\end{aligned}
$$

In order to obtain the positron (or electron) work function, one should be able to calculate the surface dipole $\Delta$. On the other hand, $\Delta$ can be eliminated when calculating the positron work function by using the experimental electron work function $\phi_{-}$,

$\phi_{+}=-\mu_{+}-\Delta=-\mu_{+}-\mu_{-}-\phi_{-}=-A_{+}-\phi_{-}$.

Thus the positron work function can also be expressed in terms of the positron affinity $\boldsymbol{A}_{+}$.

Calculated positron affinities for several elemental metals and semiconductors are shown in Fig. 24 (Puska, Lan- ki, and Nieminen, 1989). The results were obtained by using the LMTO-ASA method. The lattice structures and lattice constants used in the calculations are given in reference (Puska, Lanki, and Nieminen, 1989). The positron affinities were calculated for a smaller set of metals by Boev et al. (1987) using the LMTO-ASA method and by Farjam and Shore (1987) using the spherical cellular model. The positron affinity is also a useful quantity for compounds, such as composite semiconductors, intermetallics, carbides, and nitrides (Brauer et al., 1992; Puska et al., 1993).

The positron affinity for the elemental metals is a negative quantity, and its magnitude rises from the center of the transition-metal series towards left and right. In the case of alkali metals (bcc lattices), the distances between the positive ions are relatively large, and the small Coulomb repulsion felt by positrons in the interstitial region results in strong (large negative) positron affinities. In the group-IV semiconductors $\mathrm{Si}, \mathrm{Ge}$, and $\alpha$-Sn, the bond distances are short; but due to the diamond structure there are large open interstitial regions where the positron resides (see Fig. 1) and, as a consequence, the affinities are also strong. Alkali metals and diamondstructure semiconductors are therefore predicted to be materials from which free-positron or Ps-atom escape is not possible. Around the middle of the transition-metal series the bonding $d$-electron states are filled and the antibonding states are unoccupied. Therefore the lattice constants [bcc (left) or fcc (right) lattices] of these metals are relatively small. The positron chemical potential therefore has a high value. Because the electron chemical potential is due to the filling of the $d$ states also at a high position, the magnitude of the positron affinity is small. Thus these transition metals have a large negative positron work function, which implies that they are efficient positron moderators for the slow-positron beams.

The positron affinities have been measured directly by reemitted-positron spectroscopy (Gidley and Frieze, 1988). Moreover, the positron affinities can be deduced from the measured positronium formation potentials via

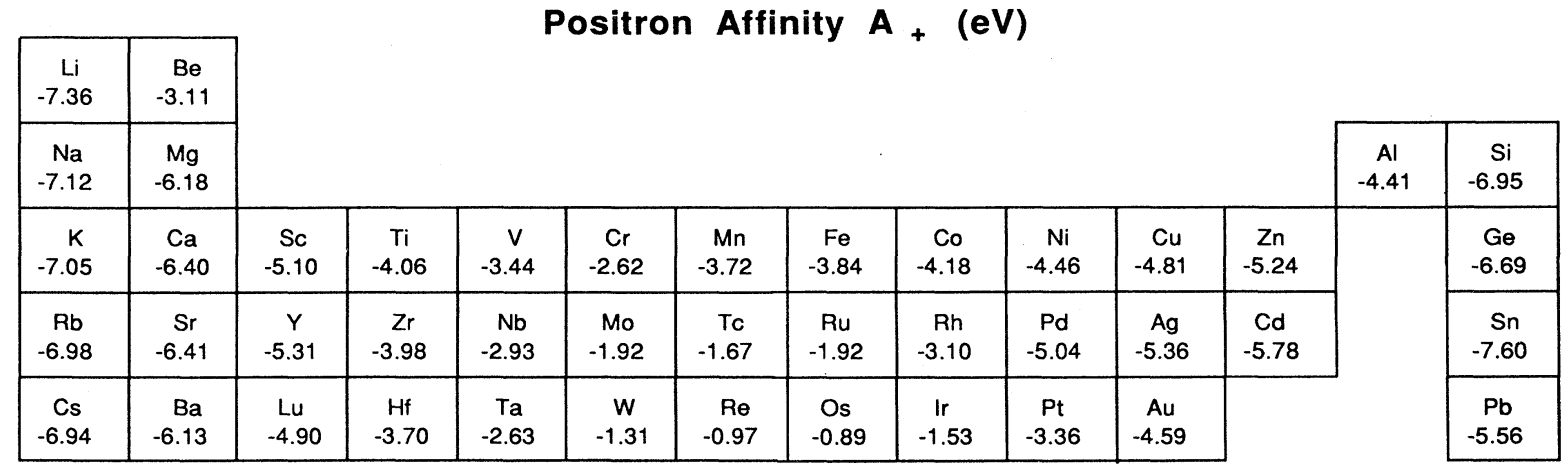

FIG. 24. Calculated positron affinities $A_{+}(\mathrm{eV})$ for elemental metals and group-IV semiconductors (from Puska, Lanki, and Nieminen, 1989). 
Eq. (116). The experimental positron affinities are compared with the measured ones in Fig. 25. This gives an idea of the reliability of the theoretical approach. The experimental and theoretical affinities obey the same trends, and the quantitative agreement is very satisfactory, considering the fact that the calculation contains no adjustable parameters. A general feature seems to be that for the bcc metals on the left-hand side of the Periodic Table the experimental affinities are slightly larger than the theoretical ones, whereas for the fcc metals on the right-hand side of the Periodic Table the situation is reversed. From the calculated positron affinities the positron work functions for different metal surfaces can be determined, with the help of Eq. (117), using experimental electron work functions. This was done by Boev et al. (1987); the results are reasonably good when compared with measured positron work functions. A more consistent way to determine the positron work functions from the calculated affinities would be the use of theoretical (Skriver and Rosengaard, 1991) values for the electron work functions.

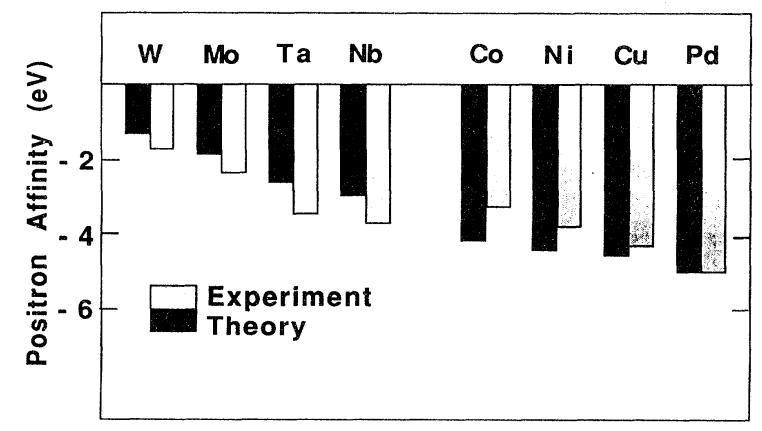

(a)

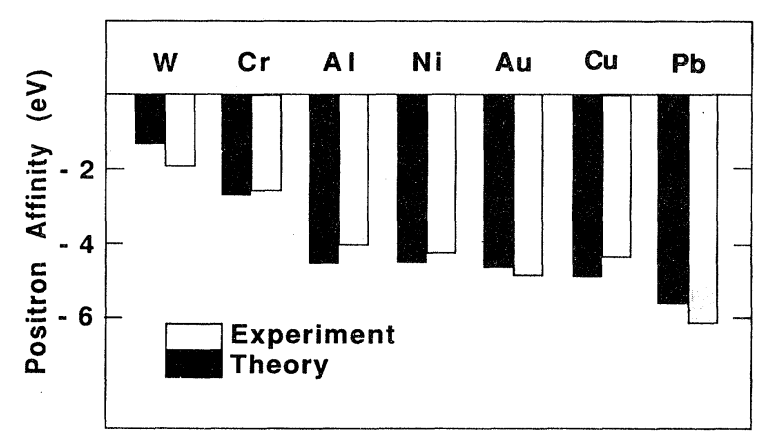

(b)

FIG. 25. Comparison of theoretical and experimental positron affinities: (a) The experimental values were measured directly using reemitted-positron spectroscopy (Gidley and Frieze, 1988). (b) The experimental values were deduced from the measured (Wilson, 1983; Wilson and Mills, 1983; Howell et al., 1987a, 1987b) positronium formulation potentials by Eq. (116). From Puska, Lanki, and Nieminen (1989).
In the case of insulating rare-gas solids, the LDA fails drastically to predict the positions of electron and positron energy levels. The electron band gap is underestimated in the LDA by approximately $50 \%$, and the positron correlation energy cannot be described as a local effect in an electron gas. It is rather due to the polarization-type deformation of the charge density of the inert-gas atoms. Recently, Puska and Nieminen (1992a) made LMTO-ASA calculations for the rare-gas solids. They solved the energy-gap problem by making a self-interaction correction (SIC; Perdew and Zunger, 1981). This means that the valence bands are shifted downwards by the same amount as the SIC lowers the valence-electron energy levels for the free atom in a LDA calculation. For the positron correlation potential, Pus$\mathrm{ka}$ and Nieminen (1992a) used the form

$$
V_{\text {corr }}(r)=-\frac{1}{2} \alpha /\left(r^{2}+r_{1}^{2}\right)^{2}
$$

where $\alpha$ is the atomic polarizability. At large distances, this equation coincides with the exact result for a point charge and a polarizable atom. At short distances, the correlation potential differs slightly from that suggested by Schrader (1979), the main improvement of Eq. (118) being the continuity of the derivative with respect to the distance. The parameter $r_{1}$ is fitted to give the measured (Gullikson et al., 1988) positron energy-band gaps at the $L$ point of the Brillouin zone. It turns out that a single value, $r_{1}=1.7 a_{0}$, can fit the band gaps within the experimental uncertainties. The correlation potential (118) is also successful in reproducing (using experimental electron work functions) the observed (Gullikson et al., 1988) trends for positron work functions for rare-gas solids.

The calculated positron affinities can be used to predict whether different types of precipitates in (dilute) alloys will trap positrons. In a simplified model the affinity difference between the precipitate and the host matrix gives the depth of a potential well sensed by the positron. When the radius of this well, i.e., that of the precipitate, exceeds the critical radius (known from basic quantum mechanics), there is a bound state for the positron at the well. There are, however, some practical difficulties in applying this scheme. The precipitates are usually not formed purely by one type of atom, but they are at least binary alloys. In order to determine the positron affinity for this alloy, one should calculate its self-consistent electronic structure and solve for the corresponding positron state (Brauer et al., 1992; Puska et al., 1993). On the other hand, if the lattice structures and lattice constants of two metals forming the precipitate alloy are not very different, a simple interpolation of the positron affinity from those of the constituents is justified (Puska, Lanki, and Nieminen, 1989). The superimposed-atom method has also been used in the context of positron trapping by precipitates or small impurity clusters in metals (Bharathi and Chakraborty, 1988). The drawback of this method is that the atomic superposition cannot describe correctly the electric dipole potential over the interface 
of the two phases. This potential can be essential for the trapping properties. On the other hand, the approach of using the positron affinities calculated for infinite bulk systems breaks clearly in the limit of small impurity clusters.

\section{Positron deformation potential}

The positron-acoustic-phonon interaction coupling constant $\gamma$ can be calculated in the deformation-potential approximation [Eq. (6)]. One need consider only longitudinal phonons because scattering off transverse modes vanishes due to symmetry. A longitudinal-acoustic phonon can be considered as successive regions with slightly larger and smaller ion densities than the equilibrium density. The derivative in the definition [Eq. (6)] of the deformation potential is calculated from the change in the (lowest) positron energy eigenvalue between two regions of different density. This energy change is affected also by the long-range Coulomb potential, which is due to electron charge transfers between different density regions and which maintains the constant chemical potential for the electrons in the sample. Therefore, in order to calculate the positron deformation potential, one needs the volume derivatives of the positron and the electron chemical potentials in a perfect, infinite crystal:

$$
E_{d}=V \frac{\partial\left(\mu_{+}\right)}{\partial V}+V \frac{\partial\left(\mu_{-}\right)}{\partial V}
$$

In terms of the positron affinity, this is given simply as

$$
E_{d}=V \frac{\partial\left(A_{+}\right)}{\partial V}
$$

The volume derivatives of the electron and positron chemical potentials and the ensuing positron deformation potential have been calculated by Boev et al. (1987) and by Farjam and Shore (1987) using modern electron band-structure methods. In the case of simple metals, the results agree qualitatively with early estimates based on the concepts of screened pseudoions (Bergersen et al., 1974). According to the simplest estimate, the deformation potential in a metal is given as $E_{d}=-\frac{2}{3} \epsilon_{F}$, where $\epsilon_{F}$ is the free-electron Fermi energy.

Soininen et al. (1990) deduced the deformation potential for several metals from diffusion coefficients measured by the slow-positron method. They used Eq. (28) with the positron effective mass of 1.5 . Their measured values for $\mathrm{Al}, \mathrm{Cu}, \mathrm{Ag}$, and $\mathrm{Mo}$ are compared with those calculated by Boev et al. (1987) in Table II. The agreement is fairly good. In addition, the measured positron diffusion coefficients for $\mathrm{Si}, 3.0 \pm 0.25 \mathrm{~cm}^{2} / \mathrm{s}$ (J. Mäkinen et al., 1990, 1991) and $2.70 \pm 0.2 \mathrm{~cm}^{2} / \mathrm{s}$ (Schultz et al., $1988)$ at $300 \mathrm{~K}$, are in good agreement with the theoretical one of $3.05 \mathrm{~cm}^{2} / \mathrm{s}$ (Boev et al., 1987). The good agreement between experimental and theoretical values gives credence to the use of the LDA in calculating positron states and energetics in solids.
TABLE II. Experimental ( $E_{d}^{\exp }$ ) (Soininen et al., 1990) and theoretical ( $E_{d}^{\text {theor }}$ ) (Boev et al., 1987) positron deformation potentials. All energies are in eV.

\begin{tabular}{ccc}
\hline \hline Host & $E_{d}^{\exp }$ & $E_{d}^{\text {theor }}$ \\
\hline $\mathrm{Al}$ & -6.7 & -7.70 \\
$\mathrm{Cu}$ & -9.4 & -9.45 \\
$\mathrm{Ag}$ & -11 & -9.48 \\
$\mathrm{Mo}$ & -16 & -14.3 \\
\hline \hline
\end{tabular}

\section{Positron bulk lifetimes}

Figure 26 (Puska, 1991a) shows the positron bulk lifetimes calculated for elemental metals and group-IV semiconductors, using the LDA described in Eqs. (83) and (85). For the metals, these are first-principles results, whereas the positron lifetimes for semiconductors are semiempirical because of the use of the experimental dielectric constants as parameters [Eq. (85)]. The selfconsistent electronic structures and the positron states are calculated using the LMTO-ASA method. In this sense these lifetimes are consistent with the positron affinities given in Fig. 24. For the simple metals, the agreement with measured lifetimes (experimental positron bulk lifetimes have been collected, for example, by Seeger et al., 1989) is very good, whereas the theoretical positron lifetimes for transition metals are consistently too low. The local-density approximation overestimates the enhancement of $d$ electrons. This seems to be the situation also in the case of II-VI compound semiconductors (Puska, 1991a) in which the cation $d$ band is close to the valence band.

The total annihilation rate $[\lambda$; Eq. (82)] calculated in the LDA can be partitioned into core $\left(\lambda_{c}\right)$ and valence $\left(\lambda_{v}\right)$ annihilation rates as suggested by Jensen (1989):

$$
\begin{aligned}
& \lambda_{c}=\int d \mathbf{r} n_{+}(\mathbf{r}) \Gamma\left(n_{-}(\mathbf{r})\right) \frac{n_{c}(\mathbf{r})}{n_{-}(\mathbf{r})}, \\
& \lambda_{v}=\int d \mathbf{r} n_{+}(\mathbf{r}) \Gamma\left(n_{-}(\mathbf{r})\right) \frac{n_{v}(\mathbf{r})}{n_{-}(\mathbf{r})},
\end{aligned}
$$

where $n_{c}$ and $n_{v}$ are the core and valence electron densities. As a matter of fact, the division of the total electron density into components with physical meaning is not, in principle, possible in the density-functional theory, but this kind of division is common practice. Further, Jensen (1989) defined the enhancement factors for the core and valence annihilations as

$$
\begin{aligned}
& \gamma_{c}=\frac{\lambda_{c}}{\lambda_{c}^{\mathrm{IPM}}}, \\
& \gamma_{v}=\frac{\lambda_{v}}{\lambda_{v}^{\mathrm{IPM}}},
\end{aligned}
$$

where $\lambda_{c}^{\text {IPM }}$ and $\lambda_{v}^{\text {IPM }}$ are the core and valence annihila- 


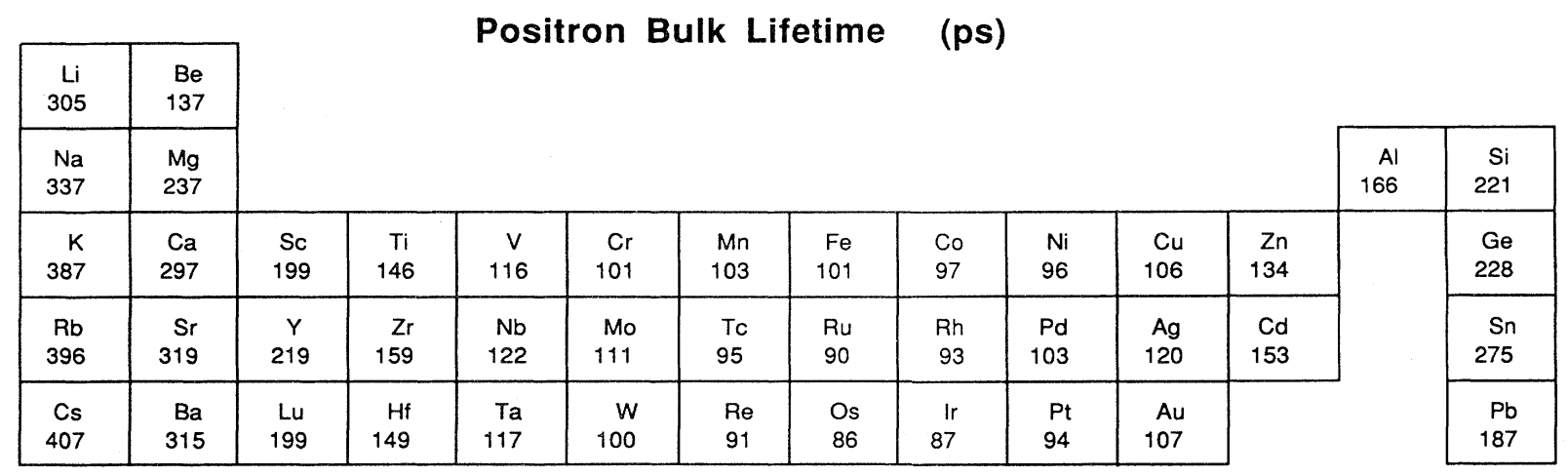

FIG. 26. Calculated positron bulk lifetimes $\tau$ (ps) for elemental metals and group-IV semiconductors (from Puska, 1991a).

tion rates in the IPM; i.e., they are calculated as in Eq. (82), but $\Gamma(n)$ is taken without enhancement as $\Gamma^{\mathrm{IPM}}(n)=\pi r_{0}^{2} c n$. In these definitions it is assumed that the enhancement factors do not depend on the energy of the electron participating in the annihilation. However, according to theory and experiments the enhancement increases as a function of the electron energy (Arponen and Pajanne, 1985). Therefore the enhancement factors [Eq. (122)] are average quantities, and, in particular, the core enhancement factors obtained in the LDA are upper estimates of the true factors. Similarly, the true core annihilation rates would be lower than those calculated from Eq. (121). Moreover, in the above equations, one should calculate the IPM annihilation rates using a positron wave function, which corresponds to the Coulomb potential only (see Sec. III.E). Bonderup et al. (1979) have presented arguments that the deep core enhancement factor should be close to unity; i.e., the IPM result holds.

For the transition metals, the core enhancement factors are typically between 2 and 3 . The valence enhancement factors are between 3 and 4 and, for the early transition metals like Sc, even larger. For the alkali metals, the enhancement factors are due to the low electron density, much larger, up to the value 25 , for valence electrons in Cs. The division into core and valence annihilation rates and enhancement factors is important for the analysis of ACAR curves. Unfortunately, the ratio between the core and valence annihilation rates cannot be found from the ACAR curves simply by fitting with a Gaussian and an inverted parabola function, because both the high-momentum components of the valenceelectron annihilation and the core annihilation contribute to the Gaussian part of the spectrum.

Jensen (1989) performed the first full LDA calculations for positron lifetimes in metals. He used non-selfconsistent electron densities and the spherical approximation. Except for the alkali metals, this does not cause large differences between his results and those shown in Fig. 26. The use of the non-self-consistent electron densities underestimates the core repulsion, increasing the core and also the valence annihilation rates. The resulting decrease in positron lifetime in alkali metals is between 10 (Li) and 25 ps (Cs). Daniuk et al. (1991) also calculated positron lifetimes from first-principles using non-self-consistent electron densities. They used for the core enhancement the zero-momentum limit of Eq. (105), whereas the valence annihilation rate was calculated according to Eqs. (82) and (83). Their lifetimes are consistently a few percent longer than those presented in Fig. 26. Sterne and Kaiser (1991) also realized the efficiency of the LDA approach in calculating positron lifetimes. However, they recommended the use of the IPM for the calculation of the core annihilation rates. The IPM gives slightly longer lifetimes for transition metals, which is in better agreement with experiments, because the too large annihilation rates with $d$ electrons are compensated by decreasing the core annihilation rate. However, this approach is hard to justify with physical arguments and can lead to practical difficulties, because it requires a sharp distinction between core and valence levels. Finally, Barbiellini, Genoud, and Jarlborg (1991) made firstprinciples calculations for positron lifetimes in solids using self-consistent electron densities. Their approach differs from the previous ones in that they do not have the correlation part in the positron potential. Moreover, they calculate the enhancement in the LDA using the approach by Jarlborg and Singh (1987; see Sec. III.E). The positron lifetimes obtained for the transition metals are longer than those shown in Fig. 26, and thereby perhaps in slightly better agreement with experiment. On the other hand, their positron lifetimes for alkali metals are much shorter than those in Fig. 26 and the experimental ones.

\section{B. Localized positron states at vacancy-type defects in solids}

The important application of positron annihilation methods in condensed matter are the numerous studies of various vacancy-type defects. The relative insensitivity 
of positrons to interstitial or substitutional defects restricts the types of defects observed. On the other hand, this often makes the analysis of the measured results much easier than that corresponding to other defectsensitive experiments, such as residual resistivity or internal friction measurements used for metals and deep-level transient spectroscopy and electron-paramagneticresonance measurements for semiconductors. However, in the case of semiconductors, negative ions at interstitial or substitutional sites can trap positrons at low temperatures (Saarinen et al., 1990). These so-called shallow traps may have considerable effects on positron annihilation characteristics. The theoretical predictions of annihilation characteristics for the defects are essential for the analysis of positron annihilation experiments, i.e., the identification of the defects. In these studies the lifetimes calculated for positrons trapped at defects are the most valuable predictions.

Most of the calculations for localized positron states at defects in solids have been based on the "conventional" scheme in which the positron does not affect the average electron density around the defect (see Sec. III.A). The full two-component description has been applied in the model system of a vacancy in jellium (spherical hole made in the rigid positive background charge for electron gas; Nieminen et al., 1985; Boroński and Nieminen, 1986), and recently in a more realistic model in which the atomic structure around the vacancy in Al is taken into account (Wang and Zhang, 1990). Calculations beyond the LDA have been performed in the case of spherical holes in jellium in order to mimic large voids in metals (Dunn et al., 1991).

\section{Results of two-component density-functional theory: vacancies in metals}

The results obtained by Boroński and Nieminen (1986) in the two-component theory for a positron trapped by a jellium vacancy are shown in Figs. 27 and 28. The jellium density and the hole radius correspond to aluminum. The positron potential and density are shown in Fig. 27 and the electron density in Fig. 28. The two-component results are compared with the functions obtained in the conventional scheme. The depth of the potential well inside the vacancy is, in both schemes, nearly the same. As a matter of fact, the potential inside the vacancy contains a constant negative component compensating the effects of the positron kinetic energy due to the ion cores which are missing in the pure jellium model. Outside the vacancy the two-component potential has a strong, long-range tail, whereas the conventional potential decays rapidly to the asymptotic value. This long-range behavior results from the cancellation of the positron self-exchangecorrelation potential and the strong electron-positron correlation contributions inside the vacancy in the twocomponent theory, whereas outside the vacancy the selfcontribution dominates. Due to the long-range potential, the positron is, in the two-component theory, slightly less

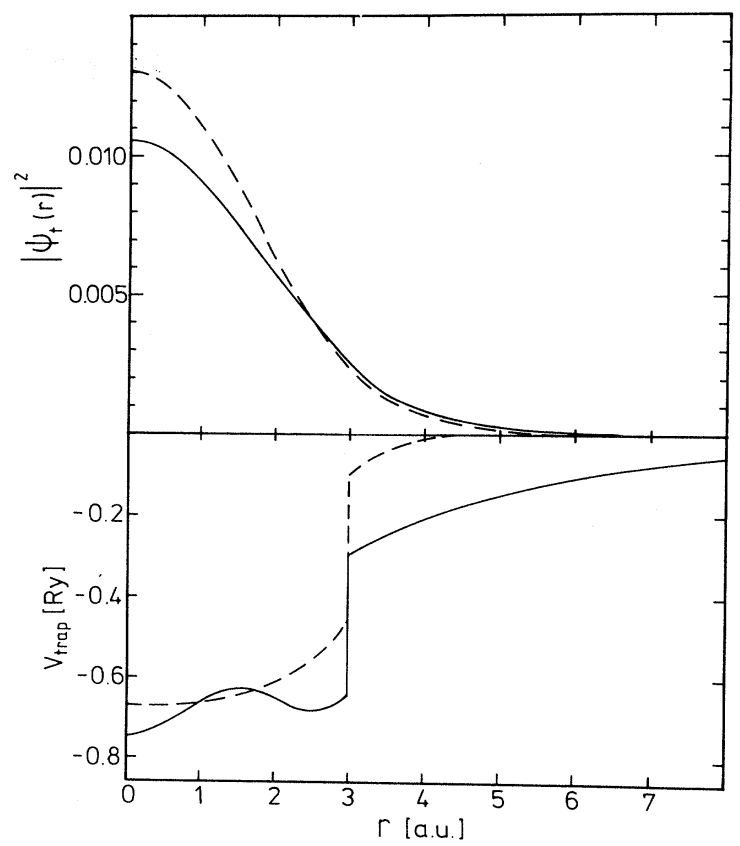

FIG. 27. Positron density $\left|\psi^{+}(\mathbf{r})\right|^{2}$ and the potential sensed by the positron in the jellium-vacancy model. The parameters of the model correspond to Al. The results of the two-component density-functional theory (solid curves) and those of the conventional scheme (dashed curves) are shown (from Boroński and Nieminen, 1986).

localized than in the conventional scheme. Figure 28 shows that the average electron density increases essentially in the presence of the positron. However, the important conclusion from the work of Boronski and Nieminen (1986) is that the relevant annihilation characteristics can be estimated reliably by the conventional scheme. For example, in the case of the aluminum vacancy, the positron binding energies are $1.87 \mathrm{eV}$ and 1.59 $\mathrm{eV}$ for the conventional and two-component schemes, re-

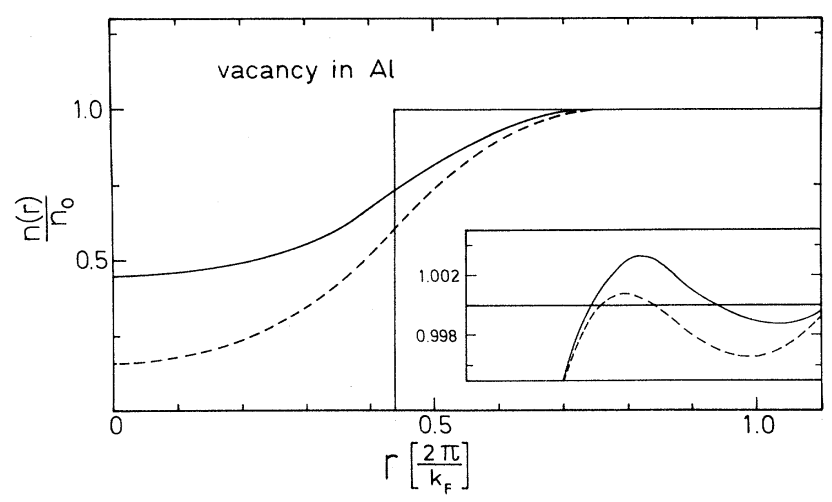

FIG. 28. Electron density at an Al vacancy in the jellium model. The results of the two-component density-functional theory in the presence of a trapped positron (solid curve) and without a positron (dashed curve) are shown (from Boroński and Nieminen, 1986). 
spectively. The corresponding positron lifetimes are 250 ps and 240 ps, respectively. Thus the differences are rather small. The insensitivity of the positron lifetime is a result of the cancellation of two effects: the average electron density increases due to the positron, but the local-contact-density-enhancement factor $g\left(0 ; n_{+}, n_{-}\right)$ (short-range screening) for a finite positron density is less than the $n_{+} \rightarrow 0$ limit used in the conventional scheme.

The positron annihilation characteristics for the vacancy in aluminum have also been calculated in twocomponent theory with a model that treats the ion cores and the lattice structure realistically (Wang and Zhang, 1990). In this calculation the embedded-cluster model (Ellis, 1979; Umrigar and Ellis, 1980) is employed within the framework of the discrete variational method (DVM; Ellis and Painter, 1970; Rosen et al., 1976; Delley and Ellis, 1982). The results obtained for the positron density and potential are very similar to those obtained in the jellium vacancy model (shown in Fig. 27). Therefore the positron binding energies and lifetimes also show the same trends as the values quoted above: the more realistic model gives the binding energies of $2.11 \mathrm{eV}$ and 1.93 $\mathrm{eV}$ for the conventional and two-component schemes, respectively (Wang and Zhang, 1990). The positron lifetimes are $244 \mathrm{ps}$ and $242 \mathrm{ps,}$ respectively. However, there is a big difference in the behavior of the electron density inside the vacancy. The jellium model predicts an increase in the average electron density due to the positron trapped into the vacancy (see Fig. 28), whereas in the discrete-ion model this increase is vanishingly small. As a matter of fact, the jellium model overestimates the electron density inside the vacancy even when there is no positron in the vacancy. The effects of the self-interaction corrections [cf. Eq. (84)] have been studied in both models. It is found that these corrections increase the positron density inside the vacancy, but the effects on the positron lifetime and binding energy are relatively small.

\section{Beyond the local-density approximation: Clean vacancies and vacancy clusters}

One of the important tasks of the positron theories for solids has been the prediction of the positron lifetimes for vacancy clusters as a function of the cluster size and possible decoration by impurities. This information has a direct bearing on the interpretation of lifetime measurements when the evolution of defect structures is investigated, for example, during annealing after particle irradiation or plastic deformation. The positron technique is most useful due to its sensitivity to vacancies and small clusters that cannot be detected by other methods, such as electron microscopy.

Positron lifetime as a function of void size was first calculated in the jellium-cavity model by Hautojärvi et al. (1977). This work was later extended by Dunn et al. (1991), who used the WDA theory of Jensen and Walker (1988; see Sec. III.C). The model parameters in both of these calculations correspond to Al. The superimposed-

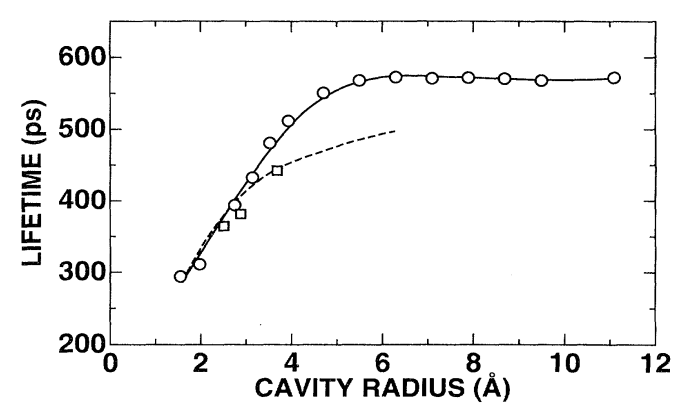

FIG. 29. Positron lifetime for voids in aluminum as a function of void radius. The solid and dashed curves correspond to the jellium-cavity model. The solid curve is a spline fit to the WDA results by Dunn et al. (1991), and the dashed curve was calculated by Hautojärvi et al. (1977) using the LDA. The squares were obtained using the superimposed-atom method in the LDA by Puska and Nieminen (1983a). The number of vacancies in the cluster is shown for the superimposed-atom results.

atom model (see Sec. III.D) has also been used to calculate (within the LDA) positron lifetimes for vacancy clusters in Al (Puska and Nieminen, 1983a). The results from the three calculations are compared in Fig. 29. The LDA results approach the value of 500 ps as the void radius increases. The atomic superposition gives, in the case of very nonspherical clusters ( 2 and 6 vacancies), clearly shorter positron lifetimes than the spherical jellium-cavity model; but for the more spherical clusters $(1,4$, and 13$)$, the results are in good agreement. In the WDA an image-like potential term appears inside the cavity for the larger clusters, similarly to the case of a plane surface (Jensen and Walker, 1988). The positron is trapped into a surface state; but because the screening cloud of the positron is always situated near the surface of the cavity, the positron lifetime approaches the longer value of 565 ps. According to Fig. 29 the LDA results are valid up to the void radius of about $3 \AA$, which corresponds to about seven vacancies in the cluster. The WDA results indicate that the saturation of the positron lifetime is complete when the void radius has grown to a value of about $9 \AA$.

\section{Vacancies and vacancy clusters decorated with impurities}

Impurity-decorated vacancies and vacancy aggregates and gas-filled voids in metals have attracted a lot of interest as systems from which new information can be gained by the positron annihilation method. Examples of the former are the carbon-vacancy pair in iron (Puska and Nieminen, 1982; Vehanen et al., 1982), nitrogenvacancy pairs in molybdenum (Nielsen et al., 1982; Hansen et al., 1984), and hydrogen-vacancy complexes in Al and in transition metals (Hautojärvi et al., 1985; Linderoth et al., 1987; Hansen et al., 1988; Rajainmäki et al., 1988). The important feature of these defects is that the impurity is located off-center in the vacancy so that there is open space in which the positron may become trapped. The positron lifetime in the vacancy- 
impurity complex is, however, shorter than that for a clean vacancy; therefore these two different defects can be separately identified. (Of course, if the two types of defects coexist in the sample, the separation of the corresponding components in the lifetime spectra may be impossible due to the small lifetime difference.) As an example, the lifetimes for a clean vacancy and for a carbon-vacancy pair in iron are 175 ps and 160 ps, respectively (Vehanen et al., 1982). Using particle irradiation followed by isochronal annealing with positron lifetime measurements, it is possible to gain information about the migration and dissociation temperatures of different defect complexes. More quantitatively, it is possible to determine from the results the impurity-vacancy binding energies. For example, the positron lifetime measurements (Rajainmäki et al., 1988) have given for hydrogen the binding energies of $0.53,0.57$, and $1.6 \mathrm{eV}$ for vacancies in $\mathrm{Al}, \mathrm{Ni}$, and $\mathrm{Mo}$, respectively. These values are in good agreement with theoretical estimations. It is also possible to analyze the actual geometry of the impurity-vacancy defects by calculating the positron lifetime for different atomic configurations using, for example, the superimposed-atom method and comparing the theoretical predictions with the measured data (Puska and Nieminen, 1982; Hansen et al., 1984).

\section{Rare-gas bubbles in metals}

Among the larger defects studied by positrons, the rare-gas bubbles in metals are the most carefully analyzed (for a review, see Nieminen, 1989). Rare-gas atoms are introduced into a metal by, for example, neutroninduced nuclear reactions. The rare-gas atoms preserve their chemically inert nature also in a metallic environment. The repulsive interaction results in the segregation of the rare-gas atoms into open volumes inside metal vacancies, voids, and grain boundaries. Especially at high concentrations the rare-gas atoms play an active role. Mobile rare-gas atoms are trapped by voids. Beyond a critical size of the agglomerate, it is energetically favorable for the precipitate to start growing (Wilson et al., 1981). This results in the appearance of rare-gas bubbles of different sizes. Within these bubbles the pressure may rise to such a high value that even heavy rare gases like $\mathrm{Kr}$ are in the solid state, even at room temperature. The rare-gas bubbles can be a serious problem for fission and fusion reactor materials, as they degrade the mechanical properties of those materials. The materials eventually show swelling, and their surfaces show blistering. The important parameters required for characterizing the rare-gas bubbles are their average size, concentration, and the gas pressure inside the bubble. As will be discussed below, positron lifetime measurements supported by theoretical predictions can give valuable information.

Jensen and Nieminen (1987a, 1987b) have made a careful theoretical analysis of the structure of rare-gas bubbles in metals and the positron states and annihilation characteristics in them. They simplified the geometry of the problem by considering a low-index surface of the metal faceting the rare-gas precipitate. This simplification is justified because the positron is in a surface state near the metal-rare-gas interface. The raregas density profiles outside the surface were first determined by molecular dynamics using proper pair potentials between two rare-gas atoms (Lennard-Jones-type pair potentials), between a rare gas and a metal atom [effective-medium theory (Manninen et al., 1984)], and between two metal atoms (semiempirical pair potentials). Figure 30 shows an example of the $\mathrm{He}$ density profiles outside the $\mathrm{Al}(100)$ surface at the temperature of $300 \mathrm{~K}$ and for two different average He densities. There is a clear peak near the metal surface, giving an average closest separation between the metal surface and the $\mathrm{He}$ atoms. At the higher density, especially, there are clear secondary peaks in the gas phase, signaling an incipient ordering. There is, however, no solidification of the $\mathrm{He}$ gas. In the case of $\mathrm{Kr}$, Jensen and Nieminen found that at high densities the $\mathrm{Kr}$ atoms order themselves to a fcc solid.

The average metal-rare-gas-atom distances obtained from molecular dynamics were used by Jensen and Nieminen $(1987 \mathrm{a}, 1987 \mathrm{~b})$ in the subsequent positron-state calculations employing the superimposed-atom method (Sec. III.D). Figure 31 shows for comparison the resulting positron wave function at an $\mathrm{Al}(100)$ surface with an ordered monolayer of $\mathrm{He}$ atoms and the state on a clean surface. The small $\mathrm{He}$ atoms make only a local disturbance in the positron wave function. Moreover, the calculations show that through the polarization interaction the $\mathrm{He}$ atoms increase the positron binding energy and thereby stabilize the surface state. The positronannihilation-rate calculations shows that the gas atoms

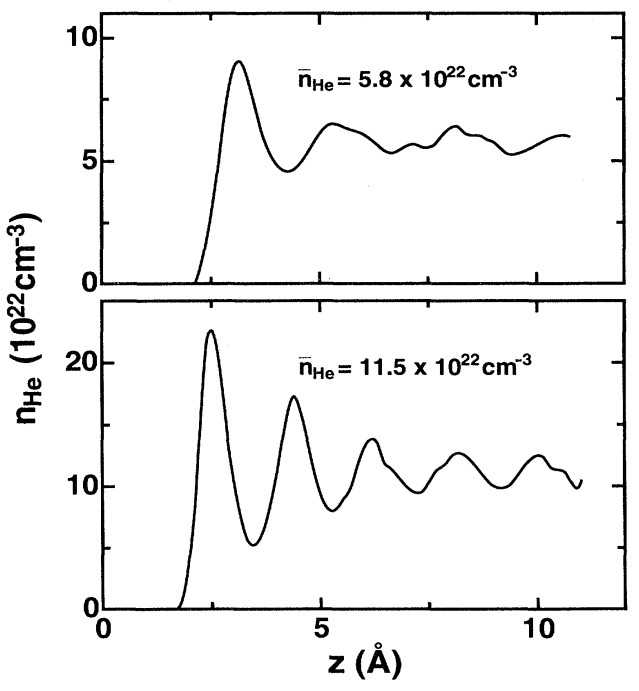

FIG. 30. He density profiles perpendicular to an $\mathrm{Al}(100)$ surface at $300 \mathrm{~K}$, calculated by using molecular dynamics. The surface $\mathrm{Al}$ atoms are at $z=0$. The profiles are shown for two different average $\mathrm{He}$ densities (from Jensen and Nieminen, 1987b). 


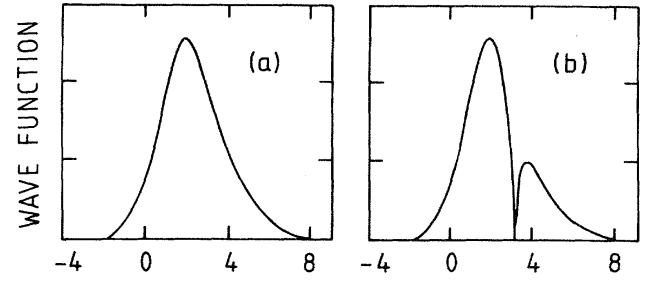

FIG. 31. Positron wave function at (a) a clean Al(100) surface and at (b) an $\mathrm{Al}(100)$ surface with an ordered monolayer of $\mathrm{He}$. The adsorbed atoms sit above the fourfold hollow sites. The cross sections shown are through a fourfold hollow site (from Jensen and Nieminen, 1987a, 1987b).

contribute additively to the total annihilation rate. Therefore, for a given density, the annihilation rate corresponding to the actual positions of the atoms at the interface can, to a good accuracy, be obtained by interpolating from the results for ordered structures. The result is a linear dependence of the positron lifetime on the rare-gas density inside the bubble. This is shown in Fig. 32 and compared with experimental results (Jensen, Eldrup, Pedersen, and Evans, 1988) in the case of $\mathrm{Kr}$ bubbles in $\mathrm{Cu}$. The $\mathrm{Kr}$ densities for the experimental results are obtained by measuring the bubble radii with a transmission electron microscope and using the equilibrium bubble pressure $(p=2 \gamma / r$, see below) and the equation of state for the different annealing temperatures. The agreement between the theoretical and experimental results is very good.

The positron lifetime results for rare-gas bubbles in metals can be analyzed to give the average bubble size and concentration. This can be done in two different ways depending on the sample conditions for positrons. In the first case the trapping into bubbles is not saturated, and both the positron lifetime at the trapped state $\tau_{b}$ and the trapping rate $\kappa$ can be determined (see Sec. II.C.1). Assuming that all the rare gas introduced into

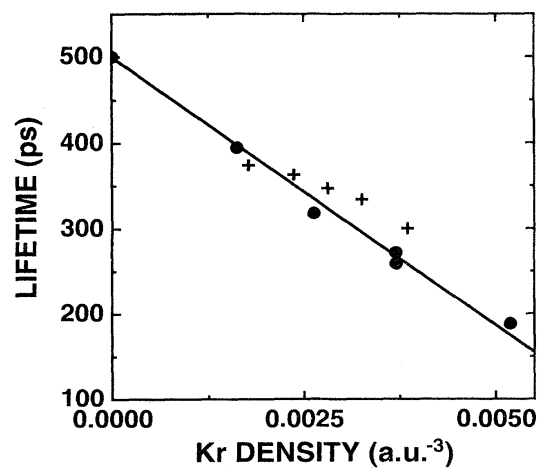

FIG. 32. Positron lifetime in a $\mathrm{Kr}$ bubble in $\mathrm{Cu}$ as a function of $\mathrm{Kr}$ density at $300 \mathrm{~K}$. The solid circles and line are the theoretical values and a fit to them, respectively (Jensen and Nieminen, 1987b). The crosses are the experimental values (Jensen, Eldrup, Pedersen, and Evans, 1988). the sample is in the bubbles, the bubble concentration is

$$
c_{t}=\frac{3 N_{g}}{4 \pi r^{3} n_{b}},
$$

where $N_{g}$ is the number of gas atoms introduced divided by the volume of the sample, $r$ is the bubble radius, and $n_{b}$ is the gas density inside the bubbles. The bubble concentration can be eliminated via $\kappa=v c_{t}$ [Eq. (32)] if the specific trapping rate $v$ is known. For example, Eldrup and Jensen (1987) used the semiempirical relation for large voids $(r>5 \AA)$,

$$
v=\left(1 / A r+1 / B r^{2}\right)^{-1} \text {, }
$$

where $A$ and $B$ are constants. Substituting the concentration $c_{t}$ in Eq. (123) and calculating the density $n_{b}$ from the measured positron lifetime $\tau_{b}$ using the linear theoretical dependence (e.g., that in Fig. 32), one can solve for the bubble radius. The radius then gives the bubble concentration via Eq. (123).

In the second case the trapping into the gas bubbles is saturated and one needs additional assumptions in the analysis. If the bubbles are in thermal equilibrium with the metal matrix, the following macroscopic relation holds between the bubble radius and the internal pressure $p$,

$$
p=\frac{2 \gamma}{r},
$$

where $\gamma$ is the surface energy of the metal. The pressure can be replaced by the gas density using the equation of state. Employing again the linear theoretical dependence between the gas density and the positron lifetime, one obtains directly the bubble radius and concentration.

In the case of small bubbles (or vacancy-impurity complexes), the positron lifetime in the trap depends both on the gas density in the trap and on the radius of the trap. The trapping coefficient is also expected to depend on these two parameters and not only on the radius. $\mathrm{Be}-$ cause these functions are not well known, the analysis of the small bubbles is difficult.

This type of analysis has been performed for $\mathrm{He}$ in $\mathrm{Al}$ (Jensen, Eldrup, Singh, and Victoria, 1988) and in $\mathrm{Ni}$ (Amarendra et al., 1992), as well as for $\mathrm{Kr}$ in $\mathrm{Cu}$ and $\mathrm{Ni}$ (Jensen, Eldrup, Pedersen, and Evans, 1988). As an example, Fig. 33 shows the radii and concentration of $\mathrm{He}$ bubbles in $\mathrm{Al}$ as a function of the annealing temperature of the sample (Jensen, Eldrup, Singh, and Victoria, 1988). The positron lifetime results are compared to those obtained using transmission electron microscopy. The results are in good agreement. Thus positron lifetime measurements provide a powerful method for characterization of gas bubbles over a wide range of bubble radii and concentrations.

Dunn et al. (1990) have calculated positron lifetime in rare-gas bubbles, preserving the spherical shape of the bubbles. This was done by using the positron pseudopotential technique for gas atoms and the jellium model for 

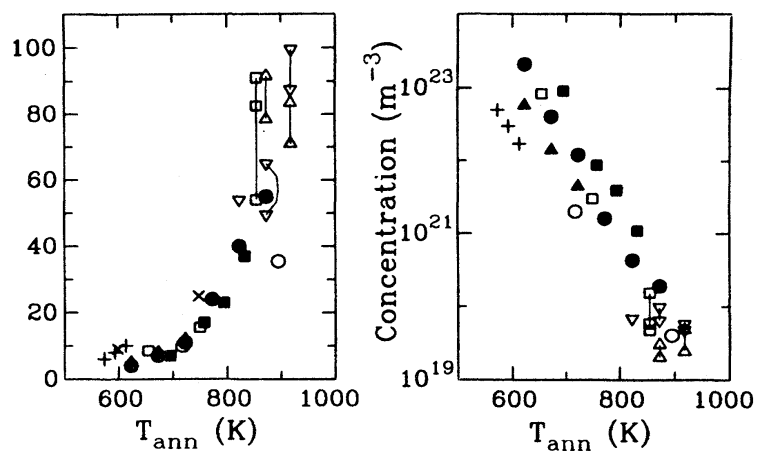

FIG. 33. Radii and concentrations of $\mathrm{He}$ bubbles in $\mathrm{Al}$ for different experimental conditions. The values correspond to bubbles annealed at $T_{a n n}$ after the introduction of $\mathrm{He}$ at lower temperatures. The solid symbols denote values from positron lifetime analysis, and the open symbols correspond to transmission electron microscope data (from Jensen, Eldrup, Singh, and Victoria, 1988).

the metal matrix. The calculations reproduce the linear dependence of the positron lifetime on the rare-gas density, in agreement with the results by Jensen and Nieminen (1987a, 1987b) for planar metal-gas interfaces.

\section{Defects in semiconductors:} electronic and ionic structures

The electronic structure of vacancy-type defects in semiconductors is much more complicated than that in metals (for reviews, see Bourgoin and Lannoo, 1983; Pantelides, 1986). A characteristic feature is the strong coupling between the electronic structure, which depends on the charge state, and the ionic configuration around the defect. For example, the strong lattice relaxation around a vacancy in silicon results in an "Anderson negative $U$ " system; i.e., the vacancy does not bind a single localized electron, but the occupancy of the deep level can change from zero to 2 (Baraff et al., 1980). Another example is served by the metastable EL2 defect in GaAs. In most of the models for EL2, atomic displacements occur as a result of electronic excitation. This is true, for example, for the model presented by Chadi and Chang (1988a) and Dabrowski and Scheffler (1989).

According to current understanding (for reviews, see Bourgoin and Lannoo, 1983; Pantelides, 1986), the relaxation of a vacancy in a semiconductor depends on the charge state of the vacancy through the number of localized electrons in the deep levels in the band gap. The deep levels in the band gap correspond to $p$-type wave functions formed by the hybridization of the four dangling bonds at the vacancy. The occupation of the deep levels depends on the position of the Fermi level. The Fermi-level positions at which the occupation changes are called the ionization levels. When there are no electrons in the deep levels, the relaxation is symmetry con- serving; i.e., there is only a breathing component in the relaxation. If there is one electron in a deep level, a tetragonal relaxation connected with the Jahn-Teller effect lowers the symmetry so that the atoms nearest to the vacancy move closer to each other: there is a pairing component in the relaxation. The second electron added can, due to the spin degeneracy, occupy the same spatial state as the first one. Therefore the symmetry does not change, but the magnitude of the relaxation is usually larger. Figure 34 shows the displacements of the atoms nearest to the vacancy in this case. The total displacement is divided into a breathing (b) and a pairing (p) component. The third bound electron has to occupy a spatially different state as the two previous ones, and it causes a further lowering of symmetry. The relaxation is a mixture of tetragonal and trigonal relaxations, and the displacements can no longer be described by the twocomponent vector scheme of Fig. 34 .

In the interpretation of the positron annihilation results for defects in semiconductors, the most important electronic and atomic structure parameters are the positions of the ionization levels in the band gap, the formation energies of the different native defects, and the positions of the atoms near the defect. These parameters are difficult to determine experimentally. However, there are several works in which the ionization levels have been determined from first-principles electronic structure calculations employing several different practical techniques and different levels of approximations. For example, the ionization levels have been calculated for vacancies, antisite defects, and self-interstitials with Green's-function methods using pseudopotentials (Baraff and Schlüter, 1985) or LMTO basis (Puska, 1989); with the tightbinding recursion method (Xu, 1990; $\mathrm{Xu}$ and Lindefelt, 1990); or with the pseudopotential supercell method (Jansen and Sankey, 1989; Jansen, 1990). All these calculations assume ideal defects; i.e., the lattice relaxation around the defect is not taken into account. Only very recently have the ionization levels been calculated allowing for lattice relaxation (Zhang and Northrup, 1991).

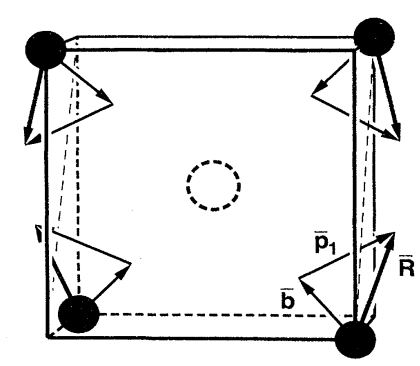

FIG. 34. Nearest-neighbor relaxations associated with a tetragonally relaxed vacancy in a semiconductor. The components of one relaxation vector are shown; $b$ denotes the breathing mode and $p$ the pairing mode. They both lie in the (110) plane shown. The directions of the vectors correspond to the negative As vacancy calculated by Laasonen et al. (1992), but the lengths are scaled by a factor of 2.5 . 
The different calculations are in qualitative agreement, but there exist some discrepancies which are important with respect to the interpolation of the positron annihilation results. For example, according to some calculations (Baraff and Schlüter, 1985; Zhang and Northrup, 1991) the As vacancy in GaAs exists only in the positive charge state, whereas other calculations (Jansen and Sankey, 1989; Puska, 1989; Xu and Lindefelt, 1990) also show the neutral and negative charge states. This is an important problem because the positive vacancy cannot bind a positron. As a matter of fact, the positron lifetime measurements have been used in a quantitative determination of the ionization levels for the As vacancy in GaAs (Corbel et al., 1988; Saarinen et al., 1991).

In order to find the equilibrium defect concentrations at finite temperatures, the defect formation energies are needed. Unfortunately, the calculation of the defect formation energies has been until recently a very difficult problem for the density-functional theory. This is because these energies are determined as small differences between the total energies of the different systems, and the total energy is sensitive to the different approximations made in the calculation of the electronic structure (Drittler et al., 1991). In the case of semiconductors, the lattice relaxation around the defect is important and should be taken into account, as is done, for example, by Zhang and Northrup (1991).

The calculation of the ionic positions, i.e., the relaxations, for a given state of the defect is a demanding task, and only very recently have these kinds of calculations been performed fully self-consistently without any pointsymmetry requirements around the defect. For example, Antonelli and Bernholc (1989) calculated the electronic and ionic structure of the vacancy in Si using pseudopotentials and a 32-atom supercell. They found that the nearest neighbors of the vacancy relax inwards towards the center of the vacancy, and the radial component of the relaxation is $2.8 \%$ of the bond length. Wang et al. (1991) also found inward relaxation for the Si vacancy using tight-binding molecular dynamics: the relaxation occurs in pairs approximately along [110] directions, as in Fig. 34, and the magnitude is very large, about $21 \%$ of the bond length. The effects of the change of the charge state were not considered in these recent studies. The inward relaxation is in disagreement with earlier theoretical predictions (Baraff et al., 1980; Scheffler et al., 1985) and with experimental results for vacancy- $P$ pairs in $\mathrm{Si}$ (Samara, 1988, 1989). The large scatter in the theoretical results reflects the difficulties in these kinds of calculations. Approximations in the potential and in the wavefunction basis sets, as well as in the finite size of the supercell, may cause large errors in the relaxations.

Recently, Laasonen et al. (1992) studied the electronic properties and the corresponding ionic relaxations of vacancies in GaAs using the so-called Car-Parrinello method (Car and Parrinello, 1985). They showed that the relaxation of the vacancy may change considerably between different charge states. The results of this work are reviewed below in the context of positron lifetime calculations (Laasonen et al., 1991), which were performed on the basis of the atomic positions obtained.

\section{Defects in semiconductors: positron states}

Before reviewing the results of positron-state calculations for defects in semiconductors, let us discuss a fundamental effect that may affect the positron annihilation in these systems (Alatalo et al., 1993). This effect is based on the fact that at the defects in semiconductors there are localized electron states which, depending on the charge state, may be partly occupied. If the occupation is an odd number, there is a localized unpaired electron and the defect has a net spin. When a positron is trapped by this kind of defect, a system resembling the Ps atom is formed. The positron and the unpaired electron may have their spins parallel (ortho state) or antiparallel (para state). Because the selection rules of quantum electrodynamics forbid the two-gamma annihilation in the ortho state, the positron annihilation in the ortho state may occur only with the spin-compensated electron density ("pickoff" annihilation). For the para state, annihilation also occurs with the unpaired electron. As a result the positron trapping at this kind of "paramagnetic" defect should lead to two lifetime components. Alatalo et al. (1993) estimated, using the electron structures from the Car-Parrinello and the LMTO-ASA Green's-function methods, the size of the effect in the case of the negatively charged vacancy in Si and in the case of the neutral As and doubly negative Ga vacancies in GaAs. The difference in the positron lifetime between the ortho and para states is of the order of $20-30 \mathrm{ps}$. This splitting is so small that the two lifetime components are expected to be mixed (averaged) in the present positron lifetime measurements. The mixing of the ortho and para components also means that the theoretical counterpart is the usual LDA annihilation rate.

The positron states in ideal vacancies in semiconductors have been calculated using both self-consistent electron densities (Puska et al., 1986; Puska, 1991a, 1991b) and the superimposed-atom method (Puska and Corbel, 1988; Puska, Mäkinen, et al., 1989; Puska, 1991a, 1991b). All these calculations employ the conventional scheme; i.e., the localized positron does not affect the average electron density. As an example of the wave functions, Fig. 2(b) shows the state in the Si vacancy. The wave function is rather delocalized as compared to similar plots for metal vacancies: there is a clear tendency for the wave function to leak into the interstitial regions. The rather weak localization is reflected in the small positron binding energies and small lifetime increase from the bulk value in semiconductors relative to metals. The lifetimes and binding energies are shown in Table III for vacancies in Si and GaAs as obtained by using the local-density approximation with the reducedscreening model [Eq. (85)]. The values (Puska, 1991a, 1991b) in the column $\tau_{\mathrm{SC}}$ were obtained by using self- 
TABLE III. Positron lifetimes for vacancies in $\mathrm{Si}$ and $\mathrm{GaAs}$. The theoretical results (from Puska, 1991a) for ideal vacancies obtained by using self-consistent $\left(\tau_{\mathrm{SC}}\right)$ and non-self-consistent $\left(\tau_{\text {NSC }}\right)$ electron structures are compared with experimental $\left(\tau^{\exp }\right)$ lifetimes. The self-consistent calculations were performed using the LMTO-ASA Green's-function method, whereas the non-self-consistent results were obtained using the threedimensional superimposed-atom method. The experimental results for the $\mathrm{Si}$ vacancies actually refer to $\mathrm{Si}$ vacancy- $P$ pairs.

\begin{tabular}{cccccc}
\hline \hline Vacancy & $\begin{array}{c}\tau^{\mathrm{SC}} \\
(\mathrm{ps})\end{array}$ & $\begin{array}{c}E_{b}^{\mathrm{SC}} \\
(\mathrm{eV})\end{array}$ & $\begin{array}{c}\tau^{\mathrm{NSC}} \\
(\mathrm{ps})\end{array}$ & $\begin{array}{c}E_{b}^{\text {NSC }} \\
(\mathrm{eV})\end{array}$ & $\begin{array}{c}\tau^{\text {exp }} \\
(\mathrm{ps})\end{array}$ \\
\hline Bulk Si & 221 & & 218 & & $220^{\mathrm{a}}$ \\
$V_{\mathrm{Si}}^{0}$ & 249 & 0.81 & 250 & 0.36 & $268^{\mathrm{b}}$ \\
$V_{\mathrm{Si}}^{-1}$ & 251 & 1.08 & & & $250^{\mathrm{b}}$ \\
Bulk GaAs & 230 & & 224 & & $232^{\mathrm{a}}$ \\
$V_{\mathrm{Ga}}^{0}$ & 262 & 1.08 & 254 & 0.44 & $260^{\mathrm{c}, \mathrm{d}}$ \\
$V_{\mathrm{Ga}}^{-1}$ & 263 & 1.26 & & & \\
$V_{\mathrm{As}}^{0}$ & 263 & 0.75 & 256 & 0.24 & $295^{\mathrm{e}, \mathrm{f}}$ \\
$V_{\mathrm{As}}^{-1}$ & 265 & 1.00 & & & $260^{\mathrm{e}, \mathrm{f}}$ \\
\hline \hline
\end{tabular}

${ }^{a}$ Experimental lifetimes quoted by Puska, Mäkinen, et al., 1989.

${ }^{\text {b} M a ̈ k i n e n, ~ H a u t o j a ̈ r v i, ~ a n d ~ C o r b e l, ~} 1992$.

${ }^{\mathrm{c}}$ Hautojärvi et al., 1986.

${ }^{\mathrm{d}}$ Corbel et al., 1990, 1992.

${ }^{\text {e}}$ Corbel et al., 1988.

fSaarinen et al., 1991.

consistent electronic structures calculated by the LMTO-ASA Green's-function method (Puska et al., 1986), whereas the values (Puska, 1991a, 1991b) denoted by $\tau_{\text {NSC }}$ were obtained by using non-self-consistent electron structures in the superimposed-atom method (Puska and Nieminen, 1983a).

Table III shows that in the case of GaAs the selfconsistent electron structures lead to somewhat longer positron lifetimes in bulk and in vacancies than the nonself-consistent ones, whereas for $\mathrm{Si}$ both methods give very similar results. This difference between $\mathrm{GaAs}$ and $\mathrm{Si}$ is due to the charge transfer from $\mathrm{Ga}$ atoms to As atoms. The dependence of the positron lifetime on the charge state is weak in the LMTO-ASA Green's-function model. This is because the changes in the lattice relaxation are ignored and because the total electron density does not change radically between the different charge states. The positron state follows the small changes in the electron density, so that the total annihilation rate is nearly unaltered. In contrast to the lifetime, the positron binding energy to the defect depends on the charge state and the self-consistency of the electron density (see Table III). According to the LMTO-ASA Green's-function calculations (Puska et al., 19.86), the binding energy depends nearly linearly on the number of the bound electrons in the deep levels. The binding energies obtained with the self-consistent electron densities are larger (by a factor of more than 2) than those from the atomic superposition method. This reflects the charge transfer from the interstitial regions to the bonds between the atoms (Puska, 1991a).

The theoretical positron lifetime for the ideal Ga va- cancy in GaAs agrees well with the measured one (Hautojärvi et al., 1986; Corbel et al., 1990, 1992). In the case of the Si vacancy and the As vacancy in GaAs, the theoretical values for the ideal vacancies are close to the shorter of the experimental lifetimes, which are connected with the more negative charge states of these vacancies. Actually, the shorter Si vacancy lifetime of 250 ps has been found only for the vacancy-P pair (Mäkinen et al., 1989; Mäkinen, Hautojärvi, and Corbel, 1992). This is not crucial, because calculations have shown (Mäkinen and Puska, 1989) that the substitution of a Si atom neighboring the vacancy by a $P$ atom (ignoring the changes in relaxation) increases the positron lifetime by only 1 ps.

To reproduce the longer experimental positron lifetimes for Si and As vacancies in Table III in the conventional LDA model for positron annihilation requires that the vacancies relax outwards. The effects of the relaxation on the positron lifetime have been studied in the superimposed-atom model (Puska and Corbel, 1988; Mäkinen and Puska, 1989). The results show that only the changes in the breathing-mode component affect the lifetime appreciably (Puska and Corbel, 1988). As a rule of thumb, one can say that in Si and GaAs the outward breathing relaxation of $1 \%$ of bond distance means an increase of $\sim 3.5 \mathrm{ps}$ in the positron lifetime. In the case of the phosphorus-vacancy (P-V) pair in Si, this means that the breathing-mode relaxation should increase from $0-5.1 \%$ of the Si bond length in the charge-state transition from $(\mathbf{P}-\mathrm{V})^{-}$to $(\mathbf{P}-\mathrm{V})^{0}$. This is in reasonable agreement with the results of the DLTS measurements performed under hydrostatic pressure (Samara, 1988, 1989). They indicate that the amplitude of the relaxation increases from 3.2-6.1\%. Even the absolute numbers are in good agreement, especially if one takes into account the fact that the deep-level transient spectroscopy measurement systematically overestimates the amplitude of the breathing-type relaxation (Samara, 1988, 1989).

The systematics of the positron annihilation characteristics has also been studied for larger vacancy clusters in Si (Puska and Corbel, 1988) and for vacancies in different III-V compound semiconductors (Puska, Mäkinen et al., 1989). In the Si divacancy the positron wave function is localized in a fashion similar to vacancies in metals. This is illustrated in Fig. 2(c). The positron lifetime for the Si divacancy is then, by a factor of 1.4 , larger than the bulk lifetime. This is close to the typical ratios of 1.4-1.6 for the vacancy and bulk lifetimes in metals. The calculations for ideal vacancies and divacancies in III-V semiconductors indicate that the positron lifetime measures mainly the extent of the open volume seen by the positron, and this open volume is more or less directly proportional to the unit-cell volume of the compound in question.

Laasonen et al. $(1991,1992)$ have studied the positron states and lifetimes for vacancies in GaAs using the calculated relaxed positions of ions around the defects. First, the electronic and ionic structures were obtained 
by the first-principles molecular-dynamics method of Car and Parrinello (1985). Laasonen et al. found that the ionic relaxation can change strongly as the charge state of the vacancy changes. The Ga vacancy relaxes, in both the neutral and the different negative charge states, slightly inwards (Laasonen et al., 1992). The neutral As vacancy has a small outward relaxation, whereas, in the case of the negative and the doubly negative As vacancies, the four nearest-neighbor $\mathrm{Ga}$ atoms move strongly towards the center of the vacancy, with a large pairing component. The rather modest relaxations for the $\mathbf{G a}$ vacancies are connected with the fact that the wave functions corresponding to the deep levels (for a neutral $\mathbf{G a}$ vacancy, there are three electrons in the deep levels) are relatively close to the valence band and have a strongly delocalized character. In the case of the neutral As vacancy, there is only one electron in the deep level, at a rather high energy. Therefore its wave function is also relatively delocalized, and, correspondingly, the relaxations are small. In the negative As vacancy, the common energy level of the two deep electrons lies approximately at the midgap, and the wave function forms clear bonds between the $\mathrm{Ga}$ atoms in two pairs around the vacancy. The states are well localized and drive a large Jahn-Teller effect. The electron densities corresponding to the deep levels in neutral $\mathrm{Ga}$ and singly negative As vacancies are shown in Fig. 35.

Using the atomic positions from the Car-Parrinello calculations, Laasonen et al. (1991) determined the positron states and lifetimes with the help of the superimposed-atom method. Laasonen et al. showed that the positron energy eigenvalue lowers more than the (elastic) electron-ion system energy rises when the breathing-mode relaxation increases from the result obtained by minimizing only the latter energy term. There is a minimum of the sum of these two energies, meaning that the localized positron can eventually push the atoms slightly outwards. It is difficult to take this effect fully self-consistently into account. It could, in principle, be done by adapting the positron state into the CarParrinello method within the two-component densityfunctional theory. Laasonen et al. modeled the selftrapping effects by increasing the positron mass by a factor of 1.5. The increase in the mass leads to a better localization of the positron wave function and thereby works in the same direction as the relaxation induced by the positron. With this "effective" positron mass, the calculated positron lifetimes for the $\mathrm{Ga}$ vacancy and for the neutral and negative As vacancies are 264, 287, and $247 \mathrm{ps}$, respectively. These numbers are in fair agreement with the experimental ones shown in Table III.

Laasonen et al. (1991) calculated, using this same description, the positron lifetime for the As antisite defect in an excited state, in which the As atom is moved from the $\mathrm{Ga}$ site towards the neighboring interstitial site. In one (Chadi and Chang, 1988a; Dabrowski and Scheffler, 1989) of the several models for the EL2 defect, this state is considered to be the metastable state to

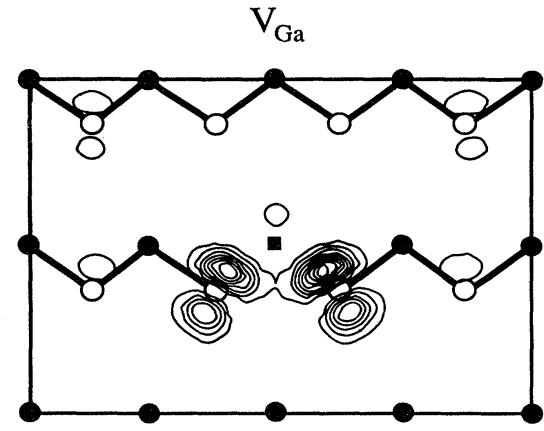

(a)

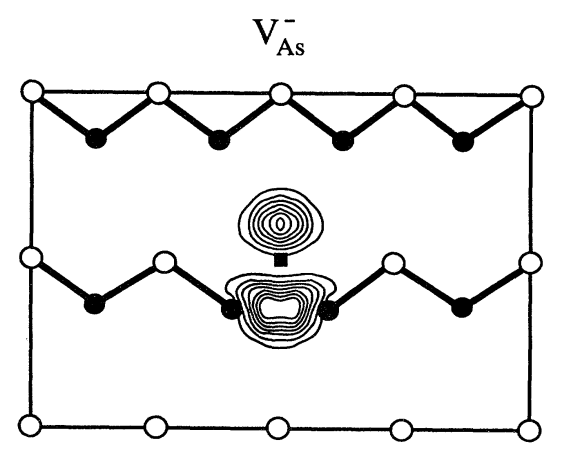

(b)

FIG. 35. Electronic density corresponding to the highest state of (a) the neutral Ga vacancy and (b) the singly negative As vacancy in GaAs. The densities are given for a (110) surface. The contour spacing is one-tenth of the maximum value. The solid circles are the $\mathrm{Ga}$ atoms, and the open ones are the As atoms. The vacancy is marked by a solid square (from Laasonen et al., 1992).

which the As antisite $\left(\mathrm{As}_{\mathrm{Ga}}\right)$ relaxes in an electronic excitation. In the metastable state the defect has an open region which is, however, smaller than a "clean" Ga vacan-

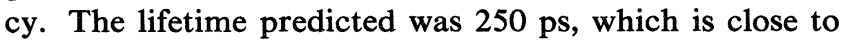
the 255 ps estimated from the measurements (Krause et al., 1990). Figure 36 shows the positron density in the case of the metastable As antisite and the different vacancies in GaAs. With the exception of the negative As vacancy, the positron states are strongly localized at the defects.

Saito et al. (1991) have calculated the momentum distribution of annihilation radiation for the perfect $\mathrm{Si}$ and GaAs lattices and for vacancies in Si using first-principles electronic structure methods. These distributions differ from the usual 2D ACAR spectra because the integration perpendicular to the plane of observation is not performed. In good agreement with experiments, the calculated distributions for perfect lattices show maxima in the [110] directions leading to valleys in the [100] directions and $\mathbf{a} \operatorname{dip}$ at the $\mathbf{k}=0$ point. Moreover, Saito et al. show, for the positrons trapped by vacancies, that the momentum distribution is isotropic with the maximum at 

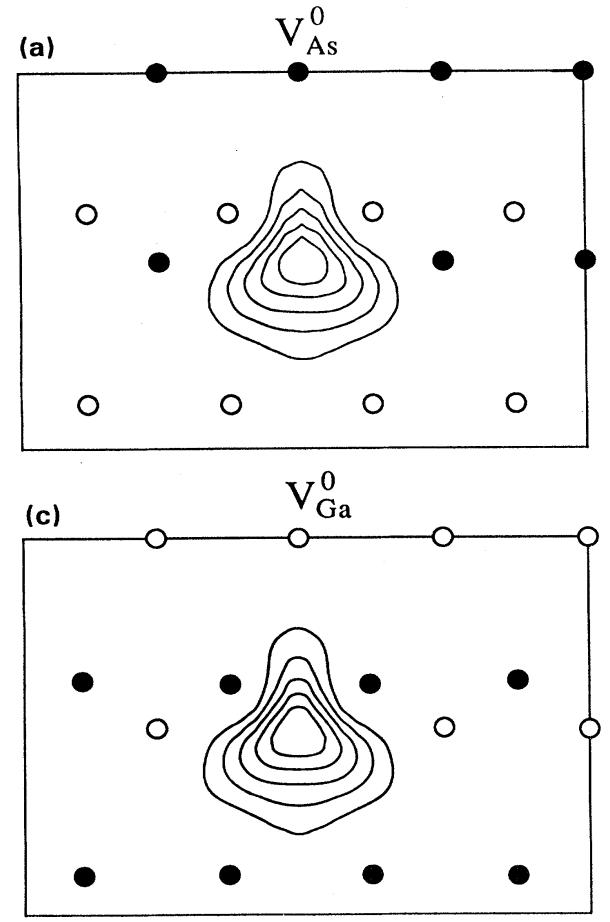
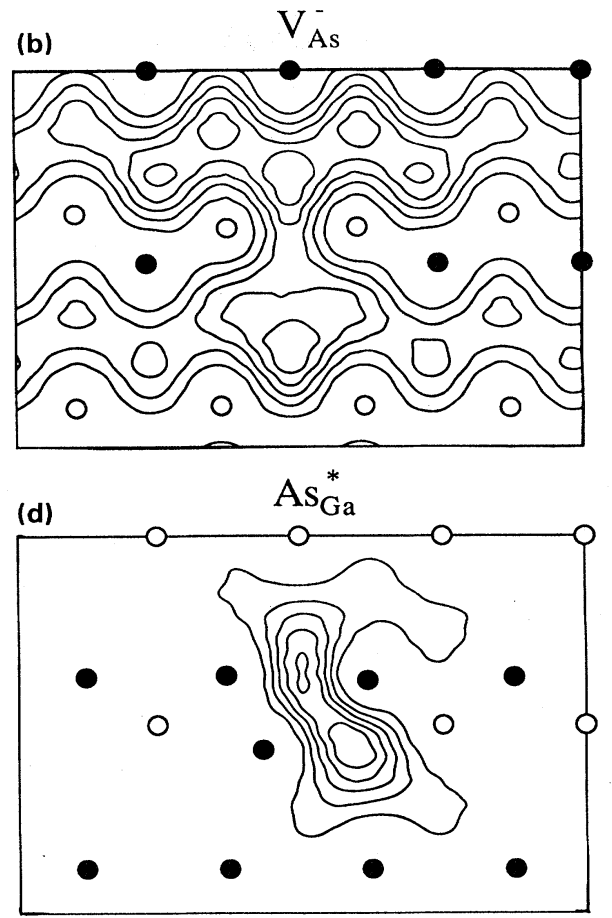

FIG. 36. Positron densities for different vacancy-type defects in GaAs: (a) neutral As vacancy, (b) singly negative As vacancy, (c) Ga vacancy, and (d) As antisite in the metastable relaxed state. The results correspond to the positron effective mass of 1.5 times the free-positron mass. A region of the (110) plane is limited by the boundaries of the supercell. The contour spacing is one-sixth of the maximum value. The solid circles are the As atoms and the open ones are the $\mathrm{Ga}$ atoms (from Laasonen et al., 1991). the $\mathbf{k}=0$ point.

The combination of positron lifetime or Dopplerbroadening measurements with the controlled optical excitation opens new possibilities for the study of defects in semiconductors. One example of this kind of work is the detection (Krause et al., 1990; Saarinen et al., 1994) of the vacancy-type defect in the metastable state of the EL2 defect in GaAs discussed above. A similar system is the DX center in AlGaAs compounds. According to a theoretical model (Chadi and Chang, 1988b), in the stable state of the DX center a donor atom, such as $\mathrm{Si}$, is in an interstitial site next to a $\mathbf{G a}$ vacancy. When the center is excited, the donor atom becomes substitutional. The existence of a $\mathrm{Ga}$ vacancy in the stable state and its disappearance after the optical ionization process have been seen recently in positron measurements (Mäkinen et al., 1993). Moreover, in a recent work, Saarinen et al. (1993) used the illumination in order to ionize As vacancies in semi-insulating GaAs to the negative charge state and observed the ionization process by positron lifetime measurements. The detailed description of the electronic and ionic structures as well as the trapped-positron states for the defect complexes studied by these new techniques is a challenge for future theoretical work.

\section{Positron states on solid surfaces}

\section{Properties of the positron surface state}

Nieminen and Puska (1983) used the superimposedatom method to describe the positron states on metal sur- faces. The image potential in the vacuum was joined to the LDA potential in the metal, using either the smooth or the corrugated mirror models as described in Sec. III.C. The calculations (Nieminen and Puska, 1983; Pus$\mathrm{ka}$ and Nieminen, 1983b) reproduce the trends in the binding energy for the different low-index surfaces, and the effects on the positron state due to surface contamination by gas atoms can be predicted. According to the model, the trapping of positrons by surface vacancies is weak; there is no clear potential well parallel to the surface, and the positron is therefore relatively free in two dimensions also in the presence of surface vacancies in the outermost layer. If the cutoff of the annihilation rate in the mirror image region is used, the calculation gives, for Al surfaces, positron lifetimes of about 650 ps (Nieminen et al., 1984), whereas the lifetimes without cutoff are of the order of 400 ps. The first direct measurement of the positron lifetime for the surface states was by Lynn et al. (1984). It gives the value of $580 \mathrm{ps.} \mathrm{This} \mathrm{measure-}$ ment, however, should be contrasted with those for cavities in metals, where the long positron lifetimes are of the order of 500 ps (Nieminen et al., 1979; Eldrup and Jensen, 1987). This corresponds to the negative positronium ion limit of the annihilation rate. More recently, Steindl et al. (1992) measured the positron lifetime on several metal surfaces using pulsed positron beam. They found in all cases two lifetime components, a short one of $\sim 100$ ps and a longer one of $\sim 400 \mathrm{ps}$. When comparing the experimental and theoretical surface-state lifetimes, one should also bear in mind that the theoretical lifetime is quite sensitive to the position of the mirror plane (Brown 
et al., 1987). Lou et al. (1988) introduced a similar cutoff model for the annihilation rate, but they defined the place of the cutoff directly in terms of the electron density. An additional source of uncertainty is the possible impurity concentration at the surface.

Jensen and Walker (1988) applied their WDA theory in the jellium surface model. The homogeneous positive background charge occupies the half space below the surface plane. The background density was chosen to mimic A1. The unperturbed electron density calculated in the LDA (Lang and Kohn, 1970) was fitted to give the experimental electron work function (Brown et al., 1987) for A1. The potential sensed by a positron was also adjusted by a step potential at the surface so that the calculations could give the experimental positron work function. The positron potential and the corresponding wave function are shown in Fig. 37. The WDA theory is able to join smoothly the LDA region inside to the mirror image region outside the metal, and a positron surface state well localized perpendicular to the surface is obtained. However, the WDA gives an incorrect asymptotic image potential, decaying as $-1 / 3 z$. The binding energy, $2.66 \mathrm{eV}$, and the positron lifetime, 599 ps, agree well with the experimental findings (Mills, 1983; Lynn et al., 1984). The form of the screening cloud $\left(n_{-}^{0}(\mathbf{r})\left[g_{d}^{h}\left(\left|\mathbf{r}-\mathbf{r}^{\prime}\right| ; n_{-}^{*}, \lambda=1\right)-1\right]\right)$ for different positron-surface separations is shown in Fig. 38. In the metal the screening cloud is isotropic around the positron,

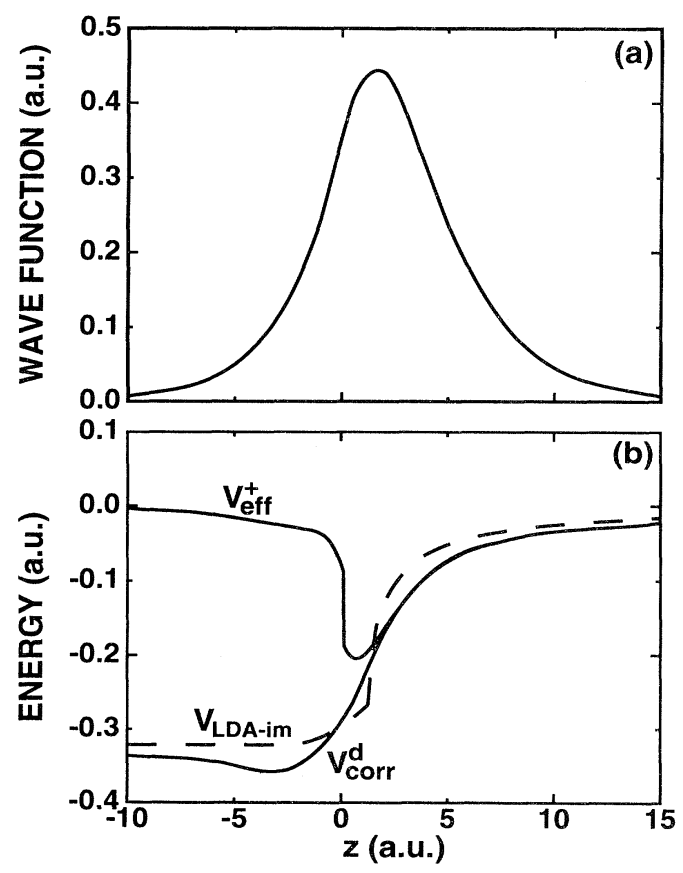

FIG. 37. Positron wave function and potential $V_{+}$for an $\mathrm{Al}$ surface in the jellium-edge model treated in the WDA by Jensen and Walker (1988). The correlation part $V_{\text {corr }}$ of the potential is also shown and compared with $V_{\text {LDA }}$, which was obtained by joining the LDA correlation potential to the $-1 / 4 z$ image potential. The jellium edge is at $z=0$ (from Jensen and Walker, 1988).

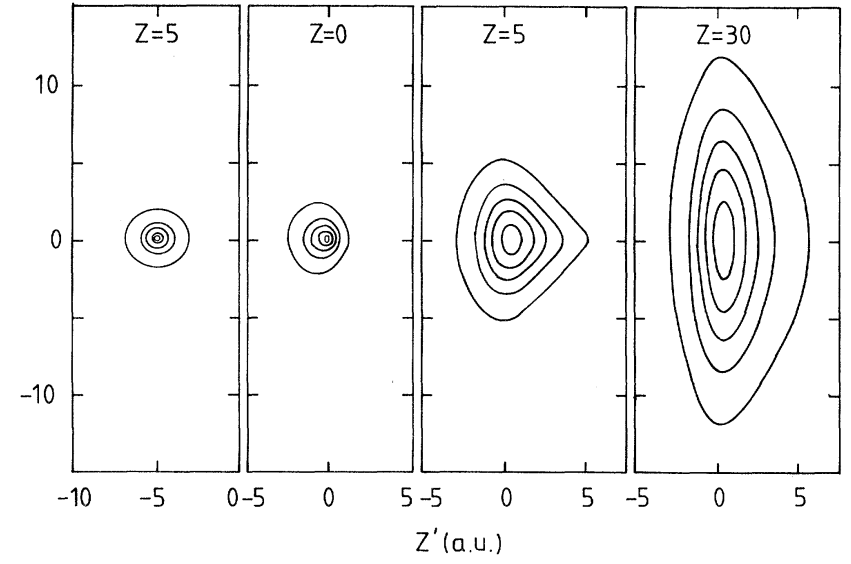

FIG. 38. Screening cloud for a positron located near the Al surface according to the jellium-edge WDA model by Jensen and Walker (1988). The different panels correspond to different positron locations. The jellium edge is at $z=0$ (from Jensen and Walker, 1988).

whereas for a positron far in the vacuum the screening cloud is left spread over a large region close to the jellium edge.

The positron state on metal surfaces has also been characterized by the 2D ACAR measurements. Figure 39(a) shows the measured (Cohen et al., 1987) momentum distribution as a contour plot with the axes along the momentum perpendicular and parallel to the surface. The distribution is nearly isotropic, which at first seems somewhat surprising. Namely, one would expect that the localization of the positron state perpendicular to the surface should weight the larger momentum components in the perpendicular directions, leaving the perpendicular distribution broader than the parallel one. This kind of anisotropic situation has indeed been found in the calculation by Brown et al. (1987), as shown in Fig. 39(b). Brown et al. used the mixed-density approximation (Sec.

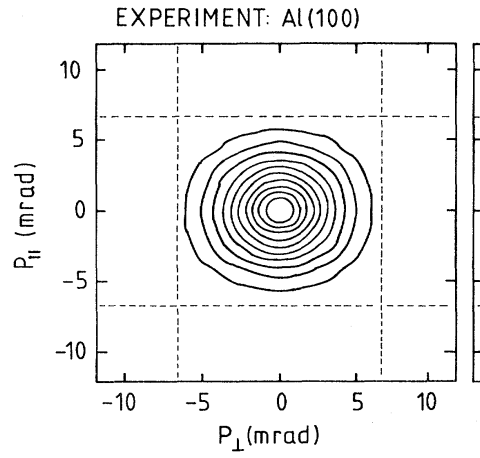

(a)

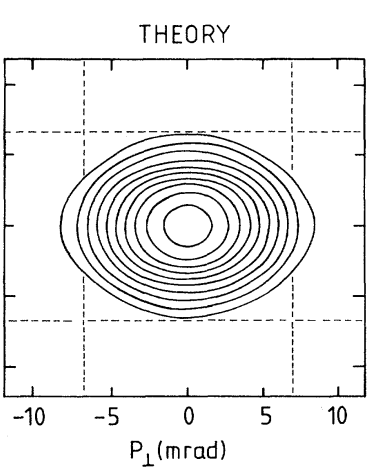

(b)
FIG. 39. Momentum distribution of the annihilating electronpositron pair for the positron state on an $\mathrm{Al}(100)$ surface: (a) experiment by Chen et al. (1987); (b) theory by Brown et al. (1987). 
III.E) with a cutoff in the local annihilation rate in the mirror image region (Sec. III.C). For the electron density, the jellium-edge model was used. The positron potential was equal to the negative of the positron work function inside the metal, but decreased smoothly, forming the potential well outside the surface, until it was replaced by the image potential. The IPM calculations by Brown et al. (1987) using the single-particle electron functions for the model potential give a more isotropic momentum distribution, but this model cannot correctly describe the positron lifetime. Brown et al. (1988) tried to remove the anisotropy by also considering the positron state localized at a vacancy on the surface in the superimposed-atom model. However, the resulting momentum distributions were still clearly anisotropic. Lou et al. (1988, 1989) have predicted a fairly isotropic momentum distribution for the electron-positron pairs annihilating on a metal surface. They used in the calculation the IPM, solved the one-particle electron wave functions for a simple model potential, and used a parametrized positron wave function. Lou et al. concluded that the most important ingredient in reproducing the experimental isotropic momentum distribution is the description of the electron distribution and work function. In their model the total electron density calculated from the wave functions shows the Friedel oscillations near the surface.

Rubaszek et al. (1985, 1991; see also Rubaszek et al., 1993) have applied the WDA and the jellium model to calculate the 2D ACAR spectra for positrons annihilating from the surface state. The WDA used differs slightly from the theory by Jensen and Walker (1988) explained in Sec. III.C. Rubaszek et al. (1993) show that the proper inclusion of positron-electron correlation effects in the calculation of the ACAR spectra is essential. They find that, in contrast to positron annihilation in bulk solids, the enhancement factor for the surface state is a decreasing function of the momentum. The resulting ACAR curves are quite isotropic and in fair agreement with experiments. Rubaszek et al. (1993) have also calculated the positron lifetimes for the surface states on several simple metals. In the case of $\mathrm{Al}$, they obtain a lifetime of 580-590 ps, in good agreement with the experimental one of 580 ps (Lynn et al., 1984).

\section{Positron-annihilation-induced Auger spectroscopy}

A positron trapped in the surface state on the solid has a finite probability of annihilating with a core electron of a surface atom. The created core hole can be filled by an electron from outer atomic shells, and the liberated energy may result in the ejection of an electron, the kinetic energy of which is measured. This process is exploited in the positron-annihilation-induced Auger spectroscopy (PAES) introduced by Weiss et al. (1988). The advantages of PAES over electron-, ion-, or x-ray-induced Auger spectroscopies are the lack of secondary electrons at the energy range of the emitted Auger electron (low background), the lack of damage caused by the exciting radiation, and the high surface sensitivity. The surface sensitivity is due to the fact that the core annihilation takes place with positrons that are trapped in the surface state and see in practice only the topmost atomic layer. In comparison, high-energy electron-excited Auger spectroscopy contains information from several atomic layers.

The central quantity for PAES is the annihilation probability with specified core electrons and the ensuing probability of the Auger process. Jensen and Weiss (1990) calculated these probabilities using the superimposed-atom method and corrugated-mirror model for the image potential. According to these calculations, which were confirmed by experiments (Mehl et al., 1990), the Auger-emission fractions are 1-5\% of the annihilations on the surface. Jensen and Weiss (1990) also showed that the probabilities are very sensitive to any adsorbate coverage, enabling the use of PAES in quantitative surface chemical analysis. Moreover, the probabilities depend on the position of the adsorbate atoms, e.g., whether they reside at a certain distance above the surface or whether they penetrate into a subsurface position. In this way PAES can also be used for structural studies of surfaces.

\section{Exotic systems}

During the last few years positron annihilation methods have been used in the characterization of novel materials soon after their invention. These cases cover the high-temperature superconductors and solid $\mathrm{C}_{60}$, the new phase of carbon. In the research of these new materials, theoretical methods have played a vital role. This is certainly true of electronic structure calculations in general. The theoretical analyses of positron states and annihilation characteristics have also been essential in the interpretation of the experimental data.

\section{High- $T_{c}$ superconductors}

The ability of the 2D ACAR measurements to give the electron momentum density with high precision has motivated the positron studies of high-temperature superconductors. The detailed momentum densities are useful in differentiating between different theoretical models for superconductivity. In the case of $\mathrm{YBa}_{2} \mathrm{Cu}_{3} \mathrm{O}_{7-\delta}(\delta=0, \ldots, 1)$, for example, self-consistent electronic structure calculations (Singh et al., 1990; Bansil et al., 1991; Barbiellini, Genoud, Henry, et al., 1991) show that there exists a Fermi surface. However, experimental confirmation of the Fermi-liquid nature through the observation of the Fermi surface by the 2D ACAR measurements has been a very difficult task. Only the very recent measurements show traces of the Fermi surface Haghigli et al., 1991a, 1991b; Smedskjaer et al., 1991, 1992; Barbiellini et al., 1993). The difficulty lies in 


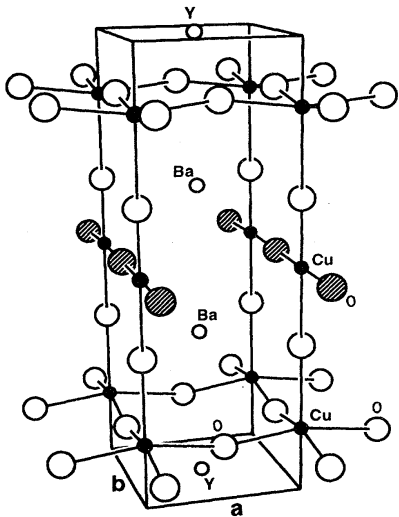

(a)

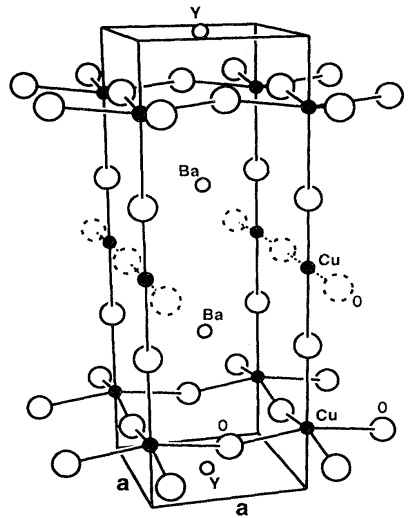

(b)
FIG. 40. Lattice structure of (a) $\mathrm{YBa}_{2} \mathrm{Cu}_{3} \mathrm{O}_{7}$ and (b) $\mathrm{YBa}_{2} \mathrm{Cu}_{3} \mathrm{O}_{6}$. The $\mathrm{Cu}-\mathrm{O}$ planes are near the top and bottom of the unit cell, and the $\mathrm{Cu}-\mathrm{O}$ chains are in the middle of the unit cell.

the fact that the Fermi-surface-related signal is only a very small fraction of the total spectrum. Moreover, it is very difficult to prepare high-quality single crystals in which positrons are not trapped by imperfections but really sample the bulk properties.

Most of the positron-state calculations have been performed for the $\mathrm{YBa}_{2} \mathrm{Cu}_{3} \mathrm{O}_{7-\delta}$ compound. The lattice structure of $\mathrm{YBa}_{2} \mathrm{Cu}_{3} \mathrm{O}_{7}$ is shown in Fig. 40(a). The lattice has orthorhombic symmetry. When $\delta>0.5$, the lattice is tetragonal, as shown in Fig. 40(b). The positron wave function for $\mathrm{YBa}_{2} \mathrm{Cu}_{3} \mathrm{O}_{7}$, calculated by McMullen et al. (1991) using the superimposed-atom method, is shown in Fig. 41. The positron density is highest between the Cu-O chains. This position is not shown in the figure. However, also on the plane shown, the maximum is close to the $\mathrm{C}-\mathrm{O}$ chain on the left-hand border of the figure, whereas, close to the C-O planes on the right-hand side of the figure, the positron density is remarkably smaller. The positron distribution determines which features of the electronic structure (which parts of the Fermi surface) might be seen in the 2D ACAR measurements.

The measured 2D ACAR data for $\mathrm{YBa}_{2} \mathrm{Cu}_{3} \mathrm{O}_{7-\delta}$ have been analyzed by Barbiellini, Genoud, Henry, et al. (1991) in order bo see the Fermi-surface-induced features. In the analysis only the anisotropic part of the data is used because the trapped positrons are expected to yield an isotropic contribution. When the full data are folded to the first Brillouin zone by the method of Lock, Crisp, and West (1973), the occupation of the electron states in the $\mathbf{k}$ space (the LCW theorem) can be given, provided the effects of the modulation of the positron wave function and enhancement in annihilation have been neglected. The filled bands produce in this analysis a constant contribution and can therefore be subtracted. Figure 42 shows the $\mathrm{LCW}$-folded distributions for $\mathrm{YBa}_{2} \mathrm{Cu}_{3} \mathrm{O}_{7-\delta}$ measured in the metallic (a) and insulator phases. The distributions can also be obtained by electron structure calculations without [(c) and (d)] and with [(e) and (f)] the effects due to positron distribution. The calculated distributions for the metallic state show clear Fermi-surfacerelated structures, sharp kinks on the surface related to the sharp change from occupied states to unoccupied ones. The experimental distributions do not, however, show similarly clear structures.

Very recently, Barbiellini et al. (1993) reported on an analysis that shows clear differences between the conducting and insulating $\mathrm{YBa}_{2} \mathrm{Cu}_{3} \mathrm{O}_{7-\delta}$ samples. Their results for the conducting and insulating systems are given in Figs. 43 and 44, respectively. The Fermi-surfacerelated feature in Fig. 43 is the ridge along the $y$ direction at $x=12.6 \mathrm{mrad}$. This ridge corresponds to the second umklapp process, for which the Fermi-surface signal is

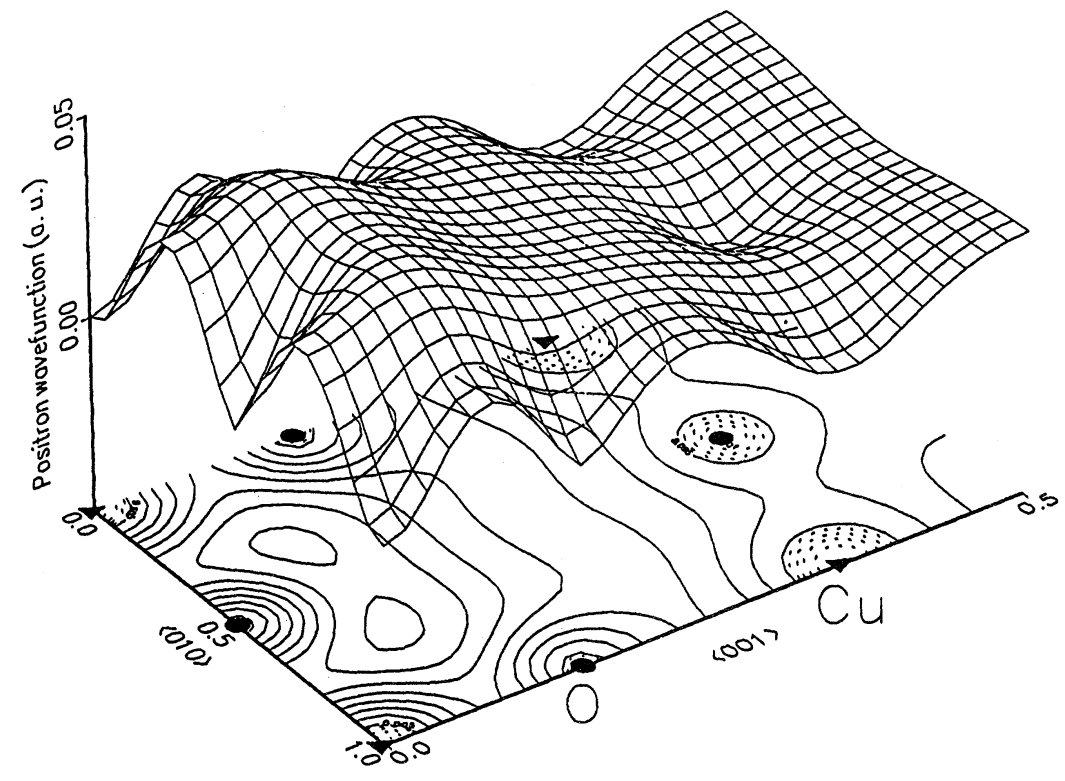

FIG. 41. Positron wave function for $\mathrm{YBa}_{2} \mathrm{Cu}_{3} \mathrm{O}_{7}$. The contours are in steps of 0.0001 (dashed lines) from 0.0001 to 0.0004 , and in steps of 0.005 (solid lines) from 0.005 to $0.04 e^{+} a_{0}^{-3 / 2}$. The normalization is one $e^{+}$ per unit cell (from McMullen et al., 1991). 


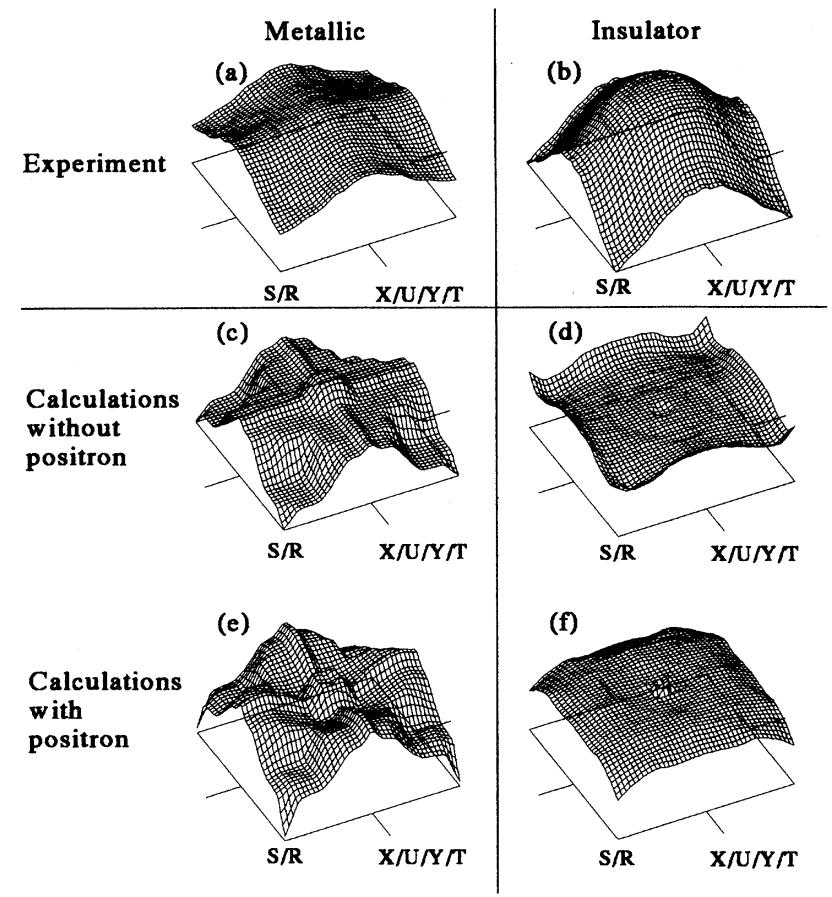

FIG. 42. Experimental $[(a)$ and (b) $]$ and calculated $[(c)-(f)]$ LCW-folded distributions for $\mathrm{YBa}_{2} \mathrm{Cu}_{3} \mathrm{O}_{7-\delta}$ (from Barbiellini, Genoud, Henry, et al., 1991).

most prominent (Haghigli et al., 1991a, 1991b). For the insulating system in Fig. 44, the ridge is absent. The theoretical results shown in Figs. 43 and 44 confirm the experimental findings.

The difficulties in seeing Fermi-surface-related features in 2D ACAR spectra have been traced to the poor quality of the samples with respect to positron states: vacancies, twin boundaries, dislocations, and grain boundaries can trap positrons and thereby change the environment and the properties of annihilation from that for the perfect bulk. The positron lifetime measurements can characterize the quality of the samples. In many cases the interpretation of the lifetime results can be based on theoretical calculations.

Jensen et al. (1989) calculated the positron states and lifetimes for $\mathrm{YBa}_{2} \mathrm{Cu}_{3} \mathrm{O}_{7-\delta}$ with $\delta=0,0.5$, and 1 using the superimposed-atom method. The positron density favors, especially in the case of $\delta=1$, the regions near the $\mathrm{Cu}-\mathrm{O}$ chains (see Fig. 41). The positron lifetime is shown in Fig. 45 as a function of the oxygen concentration and is compared with a few experimental results. The theoretical values are systematically below the experimental ones, but the results all show the same decreasing trend when the oxygen concentration increases. The inset of Fig. 45 shows that the positron affinity [the sign convention here is opposite to that in Eq. (114); i.e., a more positive affinity means stronger binding of a positron to the substance in question] decreases strongly in the same direction. This means that in samples with nonuniform stoichiometry, positrons have a strong ten- dency to localize in the oxygen-deficient zones.

According to superimposed-atom calculations (Jensen et al., 1989; McMullen et al., 1991), single oxygen vacancies in copper oxide superconductors are at best only weak traps for positrons. The binding energies are low, and the positron lifetime for oxygen vacancies exceeds only slightly the positron bulk lifetime. This kind of weak trapping, associated with detrapping, may lead to temperature dependence of lifetime spectra at low temperatures. The metal vacancies are essentially stronger traps for positrons than the oxygen vacancies. The positron lifetimes are then also substantially longer than the bulk lifetime. The picture arising from the calculations of the bulk and defect states is that the measured lifetime data reflect the competition between localization in oxygen-deficient regions and the trapping to metal vacancies. In the samples there will be, depending on the quality of the preparation, a varying but usually large number of weak traps, which lead to temperature dependences at low temperatures where they can compete as positron traps. For example, the twin boundaries from weak traps which are described in a model by McMullen et al. (1991) as oxygen vacancy-oxygen impurity pairs.

The positron-state calculations have also been performed for other copper oxide superconductors (Singh et al., 1989; Sundar et al., 1990; McMullen et al., 1991). In all materials the positron density in the delocalized state is highly nonuniform (cf. the assumptions of the LCW theorem), and in some systems the positron state may overlap more in the $\mathrm{Cu}-\mathrm{O}$ planes than in other systems. Examples of this kind of system are $\mathrm{Hg}_{2-x} \mathrm{Ba}_{x} \mathrm{CuO}_{4}$ (Singh, 1993) and $\mathrm{Nd}_{2-x} \mathrm{Ce}_{x} \mathrm{CuO}_{4}$ (Blandin et al., 1992). According to McMullen et al. (1991), in $\mathrm{La}_{2-x} \mathrm{Sr}_{x} \mathrm{CuO}_{4}$ the vacancies are only weak positron traps, and therefore the material should be a good candidate for 2D ACAR studies.

Thus far we have not discussed the effects of the superconducting state on positron annihilation. If the superconductor is BCS-like, the effects are expected to be small. The annihilation rate depends on the ThomasFermi screening wave vector $q_{\mathrm{TF}}$ as $\lambda \sim q_{\mathrm{TF}}^{d}$, where $d$ is the dimensionality of the system. Using the BCS theory, we see that the enhancement factor is proportional to the ratio between the superconducting gap $\Delta$ and the Fermi energy. This ratio is vanishingly small for regular superconductors, and the transition to the superconducting state does not affect the positron lifetime. The effects of the superconducting transition have been sought through ACAR measurements, but no evidence has been found (Barbiellini, Genoud, Henry, et al., 1991). Besides the BCS theory, other theories for high-temperature superconductivity have been considered in the context of the positron response (McMullen, 1990; Chakraborty, 1991). The results are, however, not conclusive.

\section{Fullerenes and related materials}

The innovation by Krätschmer et al. (1990) in producing the very stable $\mathrm{C}_{60}$ molecules in macroscopic quanti- 
ties has inspired a large number of experimental and theoretical studies in a short time span. In addition, positron lifetime measurements (Hasegawa et al., 1992; Jean et al., 1992; Schaefer et al., 1992a, 1992b) have been performed in order to characterize the new bulk material.
In a $\mathrm{C}_{60}$ molecule, 60 carbon atoms make a soccerball cage with the atoms at vertices of 20 hexagons and 12 pentagons. The bond lengths inside the molecule are between $1.40 \AA$ and $1.45 \AA$, similar to the bond length in graphite. At room temperature the $\mathrm{C}_{60}$ molecules form a

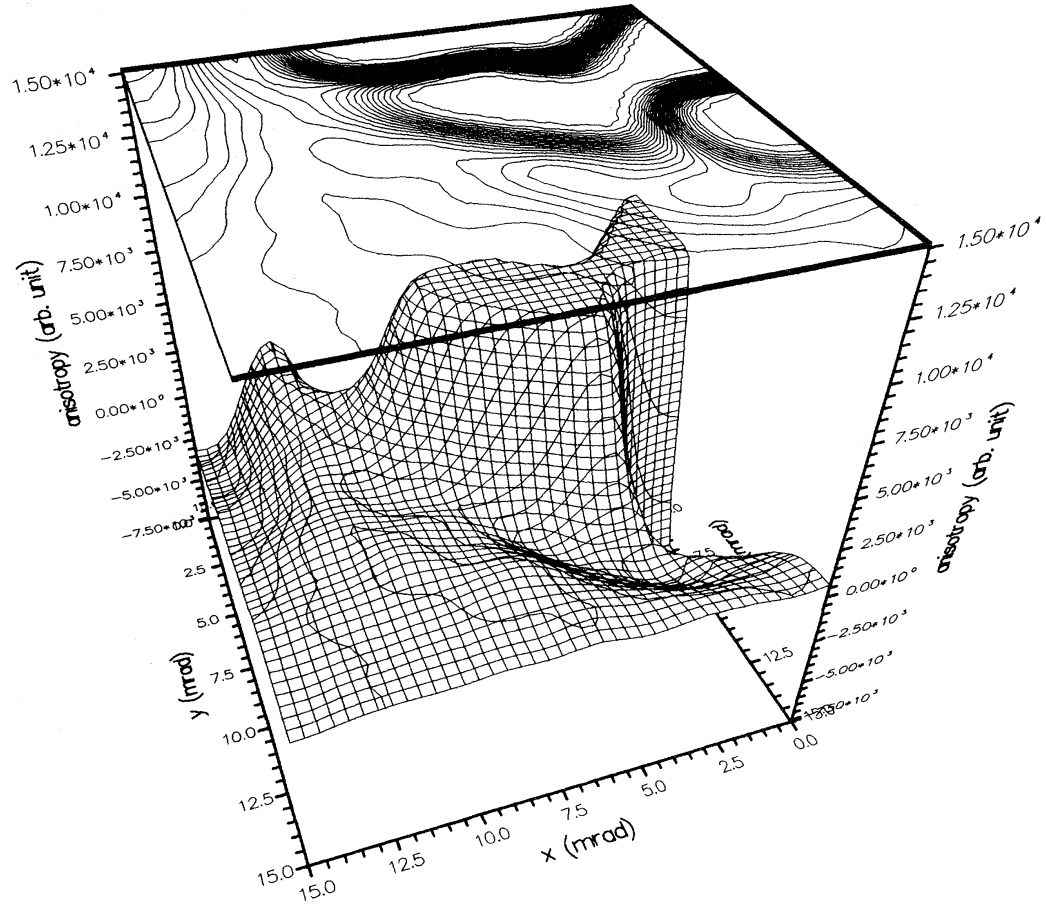

(a)

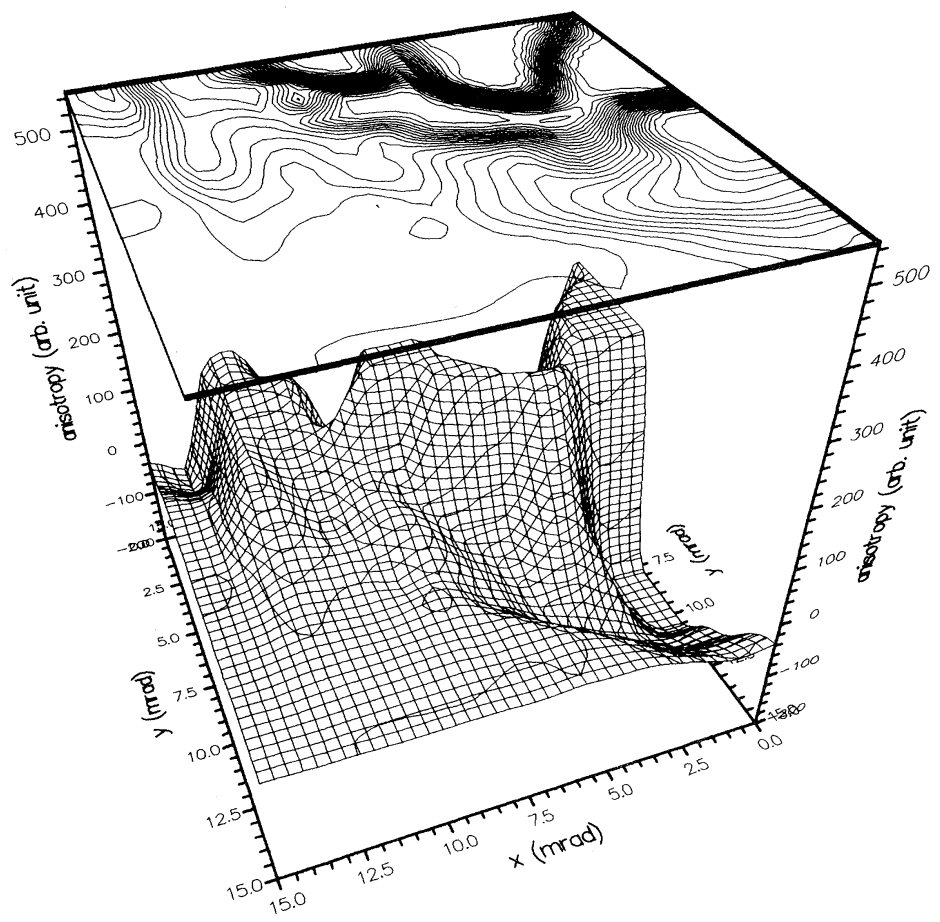

(b)
FIG. 43. Part of the 2D ACAR anisotropy for $\mathrm{YBa}_{2} \mathrm{Cu}_{3} \mathrm{O}_{7-\delta}$ : (a) measurement at $300 \mathrm{~K}$ on a sample with $T_{c}=86 \mathrm{~K}$; (b) band-structure calculation, orthorhombic structure, $\delta=0$ (from Barbiellini et al., 1993). 
fcc lattice with the lattice constant of $14.2 \AA$. The intermolecular bonds are weak van der Waals interactions, and the distance between the nearest atoms belonging to neighboring $\mathrm{C}_{60}$ molecules is rather large, 3.1 $\AA$. Figure 46 illustrates the structure of a fullerene.

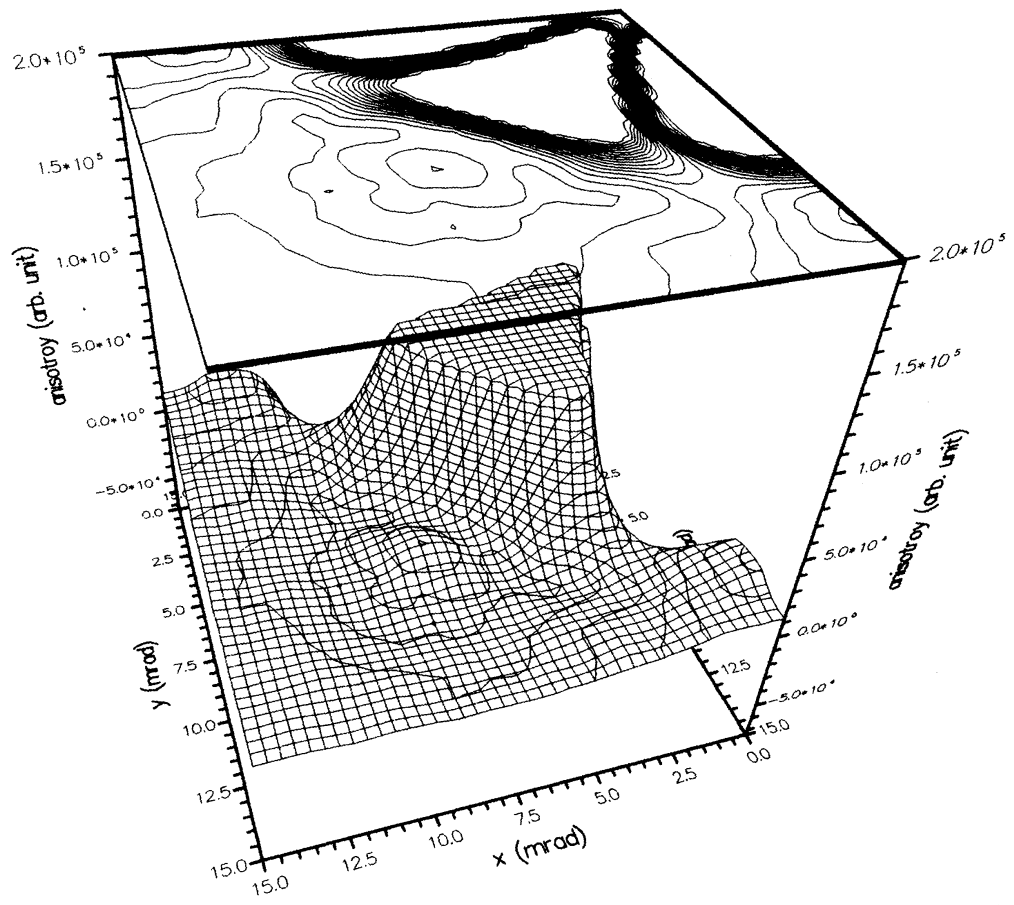

(a)

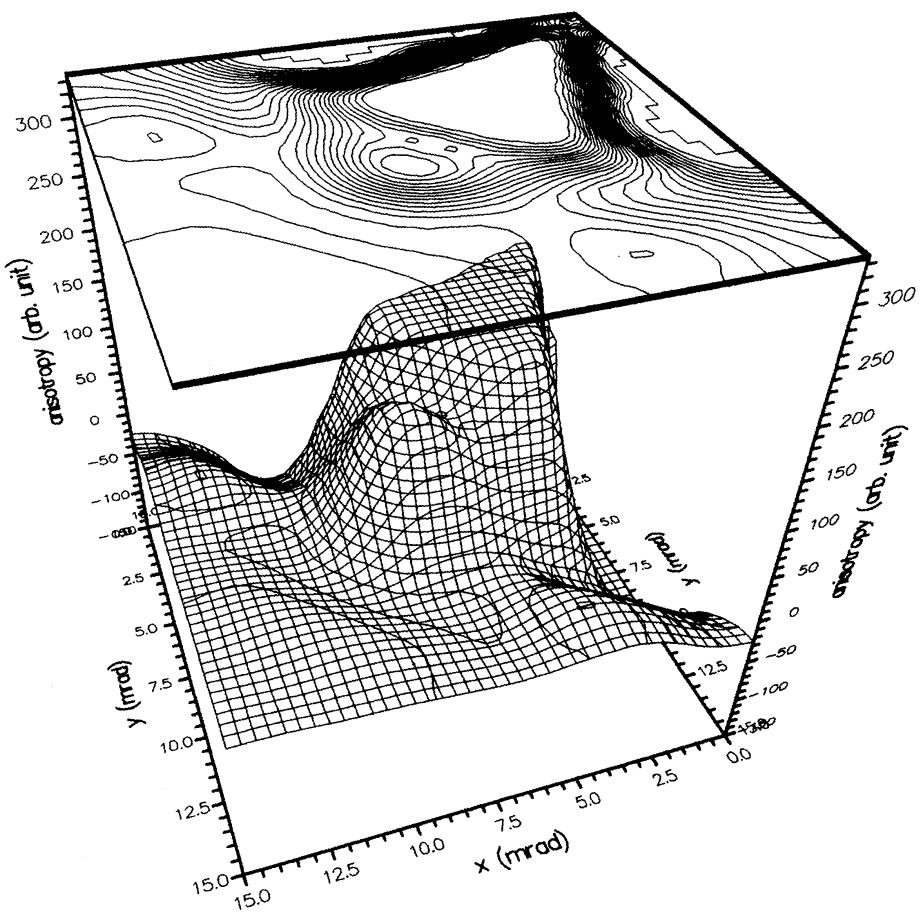

(b)
The first question concerning the interpretation of the positron lifetime data is how the positron is distributed in the fullerene material. This is of particular interest because the positron lifetime measurements show only one lifetime component of the order of 400 ps (Hasegawa
FIG. 44. Part of the 2D ACAR anisotropy for $\mathrm{YBa}_{2} \mathrm{Cu}_{3} \mathrm{O}_{6+8}$ : (a) measurement at $39 \mathrm{~K}$ on an insulating sample; (b) band-structure calculation, tetragonal structure, $\delta=0$ (from Barbiellini et al., 1993). 


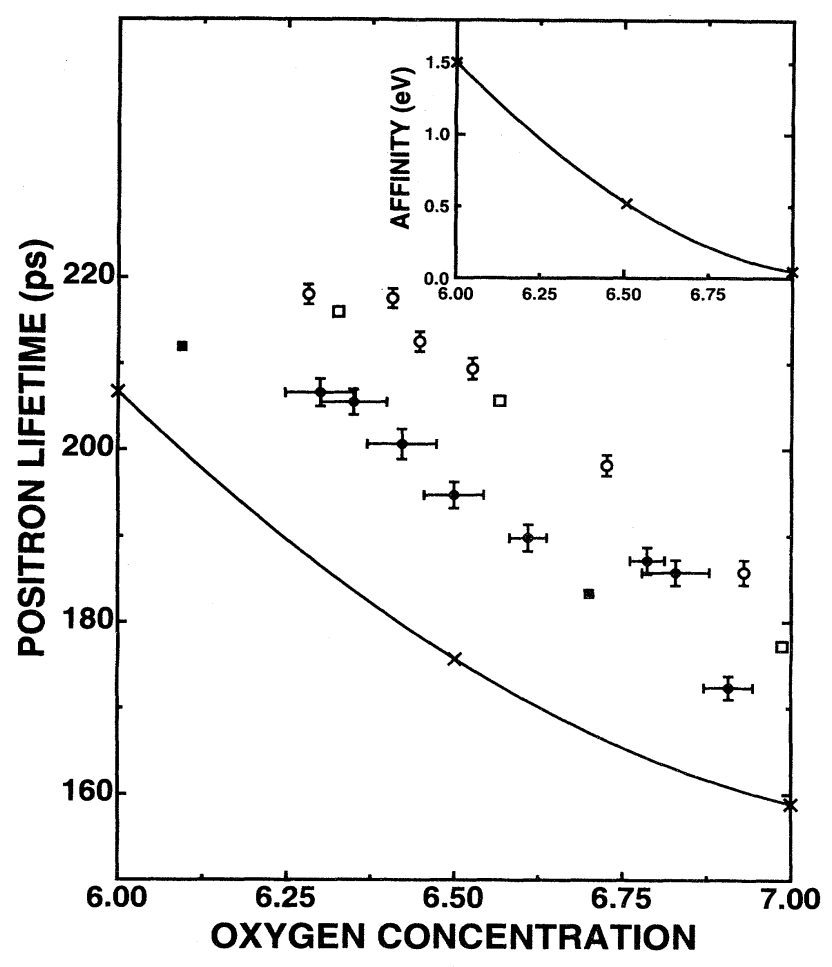

FIG. 45. Positron lifetime and affinity (with respect to the $\delta=0$ system, in the inset) for $\mathrm{YBa}_{2} \mathrm{Cu}_{3} \mathrm{O}_{7-\delta}$. The solid curves denote the theoretical results by Jensen et al. (1989); the markers denote experimental results: open circles, Smedskjaer et al. (1988); solid circles, Nyberg et al. (1989); open squares, Tang et al. (1990); solid squares, Barbiellini, Genoud, Henry, et al. (1991).

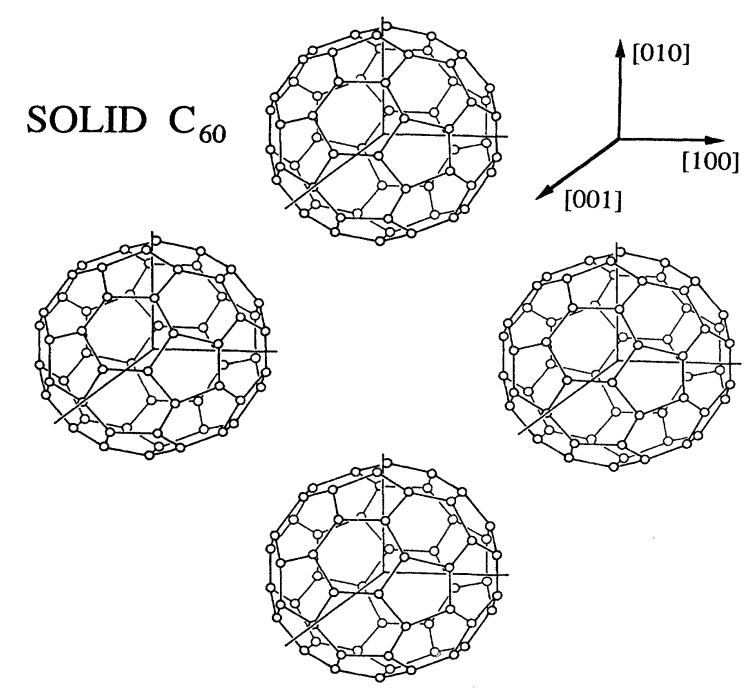

FIG. 46. Structure of a fullerene. Four $\mathrm{C}_{60}$ molecules belonging to the (001) plane of the fcc lattice are shown. The carbon atoms are at the vertices of hexagons and pentagons forming the surface of the molecules. The interatomic and intermolecular lengths are in the same scale, demonstrating the relatively large open volumes around the octahedral sites between the $C_{60}$ molecules. et al., 1992; Jean et al., 1992; Schaefer et al., 1992a, 1992b). Puska and Nieminen (1992b) calculated the positron state and lifetime in fullerene using the atomic superposition method. Similar calculations with similar results were also performed by Ishibashi et al. (1992), Jean et al. (1992), and Lou et al. (1992). The positron wave function (Puska and Nieminen, 1992b), shown in Fig. $1(d)$, is distributed throughout the interstitial regions between the balls, the maximum density being at the octahedral sites. The corresponding calculated positron life-

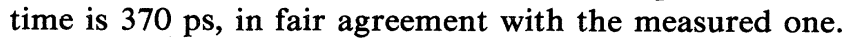
For comparison, the positron lifetimes calculated with the same method for the two more common phases of carbon, i.e., diamond and graphite, are 92 and 192 ps, respectively. Furthermore, these theoretical estimations are somewhat shorter than the measured lifetimes of 110 and $212 \mathrm{ps,}$, respectively (Schaefer et al., 1992a, 1992b). The calculations thus clearly show that the positron samples the interstitial regions in the fullerene.

Puska and Nieminen (1992b) also showed that the addition of a small concentration of dopants or interstitial impurities does not affect the positron lifetime. This is because the positron is in a highly delocalized state in fullerene and can therefore find unoccupied interstitial sites. Only when all the interstitial sites are occupied is the positron density shifted strongly toward the centers of the $\mathrm{C}_{60}$ molecules and the positron lifetime decreases. This was predicted, for example, for the superconductive $\mathrm{K}_{3} \mathrm{C}_{60}$ compound, in which all the octahedral and tetrahedral sites of the fcc lattice were occupied by potassium atoms.

\section{FINAL REMARKS}

Positron studies of condensed matter constitute an active area of research. In particular, positron-based techniques are indispensable for characterization of defects in metals and semiconductors. Positron lifetime spectroscopy is a highly sensitive technique for fingerprinting defects. Momentum density measurements, especially the 2D ACAR technique, give detailed information of the annihilating electrons. The advent of variable-energy positron beams has opened several interesting avenues in surface science. Defect profiling can be based on Doppler-broadening and Ps emission studies. Among the most interesting new methods are the positronannihilation-induced Auger spectroscopy (PAES; Weiss et al., 1988), high-resolution low-energy positron diffraction (LEPD; Horsky et al., 1992), positron microscopy (Brandes et al., 1988; Van House and Rich, 1988) and beam-based lifetime techniques (Schödlbauer et al., 1988).

We have summarized the basic physics of positron interaction with condensed matter. We have sketched the theoretical outline for the relevant processes and discussed the techniques applicable for quantitative calculations. Reliable calculations are required not only for un- 
derstanding the trends and qualitative features, but also for interpreting and analyzing the experimental results. Many of the experimental positron techniques, although unique and exceedingly accurate, give convoluted and indirect information and need strong input from theory to pin down the key parameters.

\section{ACKNOWLEDGMENTS}

We have benefited from discussions and collaboration with numerous friends and colleagues. In particular, we are grateful to Matti Alatalo, Oleg Boev, Catherine Corbel, Pekka Hautojärvi, Kjeld Jensen, Yuan Kong, Kari Laasonen, Yongming Lou, Matti Manninen, Jari Mäkinen, Kimmo Saarinen, Seppo Valkealahti, Riikka Virkkunen, and Alison Walker.

\section{REFERENCES}

In this list of references, ICPA denotes the International Conferences on Positron Annihilation held in 1985,1988 , and 1991. The proceedings of these conferences are identified by year (ICPA85, ICPA88, and ICPA91), and full information on them is given in the I's under ICPA. Similarly, SLO90 and SLO92 refer to Proceedings of International Workshops on Slow Positron Beams. Full information on them is given in the S's under SLO.

Alatalo, M., M. J. Puska, and R. M. Nieminen, 1993, J. Phys. Condens. Matter 5, L307.

Amarendra, G., B. Viswanathan, A. Bharathi, and K. P. Gopinathan, 1992, Phys. Rev. B 45, 10231.

Andersen, O. K., 1975, Phys. Rev. B 12, 3060.

Andersen, O. K., O. Jepsen, and D. Glötzel, 1985, in Highlights of Condensed-Matter Theory, edited by F. Bassani, F. Fumi, and M. P. Tosi (North-Holland, Amsterdam), p. 59.

Andersen, O. K., O. Jepsen, and M. Šob, 1987, in Electronic Band Structure and Its Applications, edited by M. Yussouff (Springer-Verlag, Heidelberg), p. 1.

Antonelli, A., and J. Bernholc, 1989, Phys. Rev. B 40, 10643.

Arponen, J., P. Hautojärvi, R. Nieminen, and E. Pajanne, 1973, J. Phys. F 3, 2092.

Arponen, J., and E. Pajanne, 1979a, Ann. Phys. (N.Y.) 121, 343.

Arponen, J., and E. Pajanne, 1979b, J. Phys. F 9, 2359.

Arponen, J., and E. Pajanne, 1985, in ICPA85, p. 21.

Asoka-Kumar, P., and K. G. Lynn, 1990, Appl. Phys. Lett. 57, 1634.

Baker, J. A., N. B. Chilton, and P. G. Coleman, 1991, Appl. Phys. Lett. 59, 164.

Baker, J. A., N. B. Chilton, K. O. Jensen, A. B. Walker, and P.

G. Coleman, 1991a, J. Phys. Condens. Matter 3, 4109.

Baker, J. A., N. B. Chilton, K. O. Jensen, A. B. Walker, and P. G. Coleman, 1991b, Appl. Phys. Lett. 59, 2962.

Bansil, A., P. E. Mijnarends, and L. Smedskjaer, 1991, Phys. Rev. B 43, 3669.

Baraff, G. A., E. O. Kane, and M. Schlüter, 1980, Phys. Rev. B 21, 5662.

Baraff, G. A., and M. Schlüter, 1985, Phys. Rev. Lett. 55, 1327. Barbiellini, B., M. Gauthier, L. Hoffmann, T. Jarlborg, A. A.
Manuel, S. Massidda, M. Peter, W. Sadowski, A. Shukla, and E. Walker, 1993, Physica C 209, 75.

Barbiellini, B., P. Genoud, J. Y. Henry, L. Hoffmann, T. Jarlborg, A. A. Manuel, S. Massidda, M. Peter, W. Sadowski, H. J. Scheel, A. Shukla, A. K. Singh, and E. Walker, 1991, Phys. Rev. B 43, 7810.

Barbiellini, B., P. Genoud, and T. Jarlborg, 1991, J. Phys. Condens. Matter 3, 7631.

Bardeen, J., and W. Shockley, 1950, Phys. Rev. 80, 72.

Bentzon, M. D., and J. H. Evans, 1990, J. Phys. Condens. Matter 2, 10165.

Bergersen, B., and T. McMullen, 1977, Solid State Commun. 24, 421.

Bergersen, B., E. Pajanne, P. Kubica, M. J. Stott, and C. H. Hodges, 1974, Solid State Commun. 15, 1377.

Bertolaccini, M., A. Bisi, G. Gambarini, and L. Zappa, 1971, J. Phys. C 4, 734.

Bethe, H. A., and J. Ashkin, 1953, in Experimental Nuclear Physics, edited by E. Segré (Wiley, New York), p. 278.

Bharathi, A., and B. Chakraborty, 1988, J. Phys. F 18, 363.

Bharathi, A., C. S. Sundar, W. Y. Ching, Y. C. Jean, P. H. Hor, Y. Y. Xue, and C. W. Chu, 1990, Phys. Rev. B 42, 10199.

Blandin, P., S. Massidda, B. Barbiellini, T. Jarlborg, P. Lerch, A. A. Manuel, L. Hoffmann, M. Gauthier, W. Sadowski, E. Walker, M. Peter, J. Yu, and A. J. Freeman, 1992, Phys. Rev. B 46, 390.

Blatt, J. M., and V. F. Weisskopf, 1952, Theoretical Nuclear Physics (Wiley, New York).

Boev, O. V., M. J. Puska, and R. M. Nieminen, 1987, Phys. Rev. B 36, 7786.

Bonderup, E., J. U. Andersen, and D. N. Lowy, 1979, Phys. Rev. B 20, 883.

Boroński, E., and R. M. Nieminen, 1986, Phys. Rev. B 34, 3820. Bourgoin, J., and M. Lannoo, 1983, Point Defects in Semiconductors II, Springer Series in Solid-State Sciences Vol. 35 (Springer-Verlag, Heidelberg).

Brandes, G. R., K. F. Canter, and A. P. Mills, Jr., 1988, Phys. Rev. Lett. 61, 492.

Brandt, W., 1967, in Positron Annihilation, edited by A. T. Stewart and L. O. Roellig (Academic, New York), p. 176.

Brandt, W., 1974, Appl. Phys. 5, 1.

Brandt, W., and N. R. Arista, 1979, Phys. Rev. A 19, 2317.

Brandt, W., and A. Dupasquier, 1983, Eds., Positron Solid-State Physics, Proceedings of the International School of Physics, Enrico Fermi, 1981, Course LXXXIII (North-Holland, Amsterdam).

Brandt, W., and R. Paulin, 1977, Phys. Rev. B 15, 2511.

Brauer, G., M. Šob, and M. J. Puska, 1992, in ICPA91, p. 611. Brenig, W., and R. Russ, 1992, Surf. Sci. 278, 397.

Britton, D., 1991, J. Phys. Condens. Matter 3, 681.

Britton, D. T., P. A. Huttunen, J. Mäkinen, E. Soininen, and A. Vehanen, 1989, Phys. Rev. Lett. 62, 2413.

Brown, A. P., K. O. Jensen, and A. B. Walker, 1988, J. Phys. F $18,1141$.

Brown, A. P., A. B. Walker, and R. N. West, 1987, J. Phys. F 17, 2691.

Car, R., and M. Parrinello, 1985, Phys. Rev. Lett. 55, 2471.

Carslaw, H. S., and J. C. Jaeger, 1959, Conduction of Heat in Solids (Clarendon, Oxford), p. 246.

Ceperley, D. M., and B. J. Adler, 1980, Phys. Rev. Lett. 45, 566. Chakraborty, B., and R. W. Siegel, 1983, Phys. Rev. B 27, 4535. Chakraborty, B., 1991, Phys. Rev. B 43, 378.

Chadi, D. J., and K. J. Chang, 1988a, Phys. Rev. Lett. 60, 2187. Chadi, D. J., and K. J. Chang, 1988b, Phys. Rev. Lett. 61, 873. 
Chen, D. M., S. Berko, K. F. Canter, K. G. Lynn, A. P. Mills, Jr., L. O. Roellig, P. Sferlazzo, M. Weinert, and R. N. West, 1987, Phys. Rev. Lett. 58, 921.

Chetty, N., K. W. Jacobsen, and J. K. Nørskov, 1991, J. Phys. C 3, 5437.

Coleman, P. G., L. Albrecht, K. O. Jensen, and A. B. Walker, 1992, J. Phys. Condens. Matter 4, 10311.

Corbel, C., F. Pierre, P. Hautojärvi, K. Saarinen, and P. Moser, 1990, Phys. Rev. B 41, 10632.

Corbel, C., F. Pierre, K. Saarinen, P. Hautojärvi, and P. Moser, 1992, Phys. Rev. B 45, 3386.

Corbel, C., M. Stucky, P. Hautojärvi, K. Saarinen, and P. Moser, 1988, Phys. Rev. B 38, 8192.

Dabrowski, J., and M. Scheffler, 1989, Phys. Rev. B 40, 10391.

Daniuk, S., G. Kontrym-Sznajd, J. Majsnerowski, M. Šob, and H. Stachowiak, 1989, J. Phys. Condens. Matter 1, 6321.

Daniuk, S., G. Kontrym-Sznajd, A. Rubaszek, H. Stachowiak, J. Mayers, P. A. Walters, and R. N. West, 1987, J. Phys. F 17, 1365.

Daniuk, S., M. Šob, and A. Rubaszek, 1991, Phys. Rev. B 43, 2580.

Darken, L. S., 1992, Phys. Rev. Lett. 69, 2839.

Delley, B., and D. E. Ellis, 1982, J. Chem. Phys. 76, 1949.

Drittler, B., M. Weinert, R. Zeller, and P. H. Dederichs, 1991, Solid State Commun. 79, 31.

Dunn, G. M., K. O. Jensen, and A. B. Walker, 1991, J. Phys. Condens. Matter 3, 2049.

Dunn, G. M., P. Rice-Evans, and J. H. Evans, 1990, J. Phys. Condens. Matter 2, 10529.

Dupasquier, A., 1992, Ed., Positron Spectroscopy of Solids, Proceedings of the International School of Physics, Enrico Fermi, 1993, Course CXXV (unpublished).

Dupasquier, A., R. Romero, and A. Somoza, 1993, Phys. Rev. B 48, 9235 .

Eldrup, M., and K. O. Jensen, 1987, Phys. Status Solidi A 102, 145.

Ellis, D. E., G. A. Benesh, and E. Byrom, 1979, Phys. Rev. B 20, 1198.

Ellis, D. E., and G. S. Painter, 1970, Phys. Rev. B 2, 2887.

Farjam, M., and H. B. Shore, 1987, Phys. Rev. B 36, 5089.

Finnis, M. W., 1990, J. Phys. Condens. Matter 2, 331.

Forster, D., 1975, Hydrodynamic Fluctuations, Broken Symmetry, and Correlation Functions (Benjamin, New York).

Foulkes, W. M., and R. Haydock, 1989, Phys. Rev. B 39, 12520.

Ghosh, V. J., K. G. Lynn, and D. O. Welch, 1993, in Positron Spectroscopy of Solids, Proceedings of the International School of Physics, Enrico Fermi, 1993, Course CXXV, edited by A. Dupasquier (unpublished).

Ghosh, V. J., D. O. Welch, and K. G. Lynn, 1992, in SLO92 (unpublished).

Gidley, D. W., and W. E. Frieze, 1988, Phys. Rev. Lett. 60, 1193.

Gleazon, G. Z., I. D. Taylor, and D. L. Tabern, 1951, Nucleon-

ics 8, 12.

Gryziński, M., 1965, Phys. Rev. 138, A305 (Part I), A322 (Part II), and A336.

Gullikson, E. M., and A. P. Mills, Jr., 1986, Phys. Rev. Lett. 57, 376.

Gullikson, E. M., A. P. Mills, Jr., and E. G. McRae, 1988, Phys. Rev. B 37, 588.

Gunnarsson, O., M. Jonson, and B. I. Lundqvist, 1979, Phys. Rev. B 20, 3136.

Haghigli, H., J. H. Kaiser, S. Rayner, R. N. West, J. Z. Liu, R.
Shelton, R. H. Howell, F. Solal, P. Sterne, and M. J. Fluss, 1991a, Phys. Rev. Lett. 67, 382.

Haghigli, H., J. H. Kaiser, S. Rayner, R. N. West, J. Z. Liu, R. Shelton, R. H. Howell, F. Solal, P. Sterne, and M. J. Fluss, 1991b, J. Phys. Chem. Solids 52, 382.

Häkkinen, H., S. Mäkinen, and M. Manninen, 1989, Europhys. Lett. 9, 809.

Hansen, H. E., R. M. Nieminen, and M. J. Puska, 1984, J. Phys. F 14, 1299

Hansen, H. E., R. Talja, H. Rajainmäki, H. K. Nielsen, B. Nielsen, and R. M. Nieminen, 1985, Appl. Phys. A 36, 81.

Harris, J., 1985, Phys. Rev. B.31, 1770.

Hasegawa, M., M. Kajino, H. Kuwahara, E. Kuramoto, M. Takanaka, and S. Yamaguchi, 1992, in ICPA91, p. 1041.

Hautojärvi, P., 1979, Ed., Positrons in Solids, Topics in Current Physics No. 12 (Springer-Verlag, Berlin).

Hautojärvi, P., J. Heiniö, M. Manninen, and R. M. Nieminen, 1977, Philos. Mag. 35, 973.

Hautojärvi, P., H. Huomo, M. J. Puska, and A. Vehanen, 1985, Phys. Rev. B 32, 4326.

Hautojärvi, P., P. Moser, M. Stucky, C. Corbel, and F. Plazaola, 1986, Appl. Phys. Lett. 48, 809.

Hehenkamp, Th., W. Berger, J. E. Kluin, Ch. Lüdecke, and J. Wolff, 1992, Phys. Rev. B 45, 1988.

Hodges, C. H., 1970, Phys. Rev. Lett. 25, 284.

Hohenberg, P., and W. Kohn, 1964, Phys. Rev. 136, B864.

Horsky, T. N., G. R. Brandes, K. F. Canter, C. B. Duke, A. Paton, D. L. Lessor, and A. P. Mills, Jr., 1992, Phys. Rev. B 46, 7011.

Van House, J., and A. Rich, 1988, Phys. Rev. Lett. 61, 488.

Howell, R. H., I. J. Rosenberg, M. J. Fluss, R. E. Goldberg, and R. B. Laughlin, 1987, Phys. Rev. B 35, 5303.

Howell, R. H., I. J. Rosenberg, P. Meyer, and M. J. Fluss, 1987, Phys. Rev. B 35, 4555.

Huomo, H., A. Vehanen, M. D. Bentzon, and P. Hautojärvi, 1987, Phys. Rev. B 35, 8252.

Huttunen, P. A., A. Vehanen, and R. M. Nieminen, 1989, Phys. Rev. B 40, 11923.

Hyodo, T., T. McMullen, and A. T. Stewart, 1984, Can. J. Phys. 62, 297.

Hyodo, T., T. McMullen, and A. T. Stewart, 1986, Phys. Rev. B 33, 3050.

ICPA85, Positron Annihilation, Proceedings of the 7th International Conference on Positron Annihilation, New Delhi, 1985, edited by P. C. Jain, R. M. Singru, and K. P. Gopinathan (World Scientific, Singapore, 1985).

ICPA88, Positron Annihilation, Proceedings of the 8th International Conference on Positron Annihilation, Gent, 1988, edited by L. Dorikens-Vanpraet, M. Dorikens, and D. Segers (World Scientific, Singapore, 1989).

ICPA91, Positron Annihilation, Proceedings of the 9th International Conference on Positron Annihilation, Szombathely, 1991, edited by Zs. Kajcsos and Cs. Szeles, Materials Science Forum 105-110 (Trans Tech Publications, Aedermannsdorf, 1992).

Ishibashi, S., N. Terada, M. Tokumoto, N. Kinoshita, and H. Ihara, 1992, J. Phys. Condens. Matter 4, L169.

Ishii, A., 1992, Ed., Positrons at Metallic Surfaces, Solid State Phenomena 28/29 (Trans Tech Publications, Aedermannsdorf).

Jacobsen, K. W., J. K. Nørskov, and M. J. Puska, 1987, Phys. Rev. B 35, 7423.

Jansen, R. W., 1990, Phys. Rev. B 41, 7666.

Jansen, R. W., and O. F. Sankey, 1989, Phys. Rev. B 39, 3192. 
Jarlborg, T., and A. K. Singh, 1987, Phys. Rev. B 36, 4660.

Jean, Y. C., X. Lu, Y. Lou, A. Bharathi, C. S. Sundar, and Y. Lyu, 1992, Phys. Rev. B 45, 12126.

Jensen, K. O., 1989, J. Phys. Condens. Matter 1, 10595.

Jensen, K. O., M. Eldrup, N. J. Pedersen, and J. H. Evans, 1988, J. Phys. F 18, 1703.

Jensen, K. O., M. Eldrup, B. Singh, and M. Victoria, 1988, J. Phys. F 18, 1069.

Jensen, K. O., and R. M. Nieminen, 1987a, Phys. Rev. B 35, 2087.

Jensen, K. O., and R. M. Nieminen, 1987b, Phys. Rev. B 36, 8219.

Jensen, K. O., R. M. Nieminen, and M. J. Puska, 1989, J. Phys. Condens. Matter 1, 3727.

Jensen, K. O., and A. B. Walker, 1988, J. Phys. F 18, L277.

Jensen, K. O., and A. B. Walker, 1990, J. Phys. Condens. Matter 2, 9757.

Jensen, K. O., and A. B. Walker, 1993, Surf. Sci. 292, 83.

Jensen, K. O., and A. Weiss, 1990, Phys. Rev. B 41, 3928.

Jones, R. O., and O. Gunnarsson, 1989, Rev. Mod. Phys. 61, 689.

Jorch, H. H., K. G. Lynn, and T. McMullen, 1984, Phys. Rev. B 30, 93.

Kahana, S., 1960, Phys. Rev. 117, 123.

Kahana, S., 1963, Phys. Rev. 129, 1622.

Kimball, G. E., and G. H. Shortley, 1934, Phys. Rev. 45, 815.

Kluin, J. E., and Th. Hehenkamp, 1991, Phys. Rev. B 44, 11597.

Kohn, W., and N. Rostoker, 1954, Phys. Rev. 94, 111.

Kohn, W., and L. J. Sham, 1965, Phys. Rev. 140, A1133.

Kong, Y. R., M. Nieminen, P. A. Huttunen, A. Vehanen, and J.

Mäkinen, 1990, in SLO90, p. 91.

Korringa, J., 1947, Physica 13, 392.

Krätschmer, W., L. D. Lamb, K. Fostiropoulos, and D. R. Huffman, 1990, Nature (London) 347, 354.

Krause, R., K. Saarinen, P. Hautojärvi, A. Polity, G. Gärtner, and C. Corbel, 1990, Phys. Rev. Lett. 65, 3329.

Kubica, P., B. T. A. McKee, A. T. Stewart, and M. J. Stott, 1975, Phys. Rev. B 11, 11.

Kubica, P., and A. T. Stewart, 1983, Can. J. Phys. 61, 971.

Kubica, P., and M. J. Stott, 1974, J. Phys. F 4, 1969.

Laasonen, K., M. Alatalo, M. J. Puska, and R. M. Nieminen, 1991, J. Phys. Condens. Matter 3, 7217.

Laasonen, K., R. M. Nieminen, and M. J. Puska, 1992, Phys. Rev. B 45, 4122.

Lang, N. D., and W. Kohn, 1970, Phys. Rev. B 1, 4555.

Lantto, L. J., 1987, Phys. Rev. B 36, 5160.

Lax, M., 1960, Phys. Rev. 119, 1502.

Lee-Whiting, G. E., 1955, Phys. Rev. 97, 1557.

Linderoth, S., H. Rajainmäki, and R. M. Nieminen, 1987, Phys. Rev. B 35, 5524.

Lock, D. G., V. H. C. Crisp, and R. N. West, 1973, J. Phys. F 3, 561.

Lock, D. G., and R. N. West, 1975, Appl. Phys. 6, 249.

Lou, Y., B. Gu, J. Zhu, C. Lee, and J. Xiong, 1988, Phys. Rev. B 38, 9490.

Lou, Y., B. Gu, J. Zhu, C. Lee, and J. Xiong, 1989, J. Phys. Condens. Matter 1, 2977.

Lou, Y., X. Lu, W. Y. Ching, Y.-Y. Xu, M.-Z. Huang, Y. C. Jean, R. L. Meng, P. H. Hor, and C. W. Chu, 1992, Phys. Rev. B 46, 2644.

Loucks, T. L., 1966, Phys. Rev. 144, 504.

Lundqvist, S., and N. H. March, 1983, Eds., Theory of the Inhomogeneous Electron Gas (Plenum, New York).
Lynn, K. G., W. E. Frieze, and P. J. Schultz, 1984, Phys. Rev. Lett. 52, 1137.

Lynn, K. G., T. McKay, and B. Nielsen, 1987, Phys. Rev. B 36, 7107.

Lynn, K. G., and B. Nielsen, 1987, Phys. Rev. Lett. 58, 81.

Makhov, A. F., 1961, Sov. Phys. Solid State 2, 1934, 1942, 1945.

Mäkinen, J., C. Corbel, P. Hautojärvi, and D. Mathiot, 1991, Phys. Rev. B 43, 12114.

Mäkinen, J., C. Corbel, P. Hautojärvi, P. Moser, and F. Pierre, 1989, Phys. Rev. B 39, 10162.

Mäkinen, J., C. Corbel, P. Hautojärvi, A. Vehanen, and D. Mathiot, 1990, Phys. Rev. B 42, 1750.

Mäkinen, J., P. Hautojärvi, and C. Corbel, 1992, J. Phys. Condens. Matter 4, 5137.

Mäkinen, J., T. Laine, K. Saarinen, P. Hautojärvi, C. Corbel, V. M. Airaksinen, and P. Gipart, 1993, Phys. Rev. Lett. 71, 3154.

Mäkinen, J., S. Palko, J. Martikainen, and P. Hautojärvi, 1992, J. Phys. Condens. Matter 4, L503.

Mäkinen, S., and M. J. Puska, 1989, Phys. Rev. B 40, 12523.

Mäkinen, S., H. Rajainmäki, and S. Linderoth, 1990, Phys. Rev. B 42, 11166.

Manninen, M., and R. M. Nieminen, 1981, Appl. Phys. A 26, 93.

Manninen, M., J. K. Nørskov, M. J. Puska, and C. Umrigar, 1984, Phys. Rev. B 29, 2314.

Martin, T., R. Bruinsma, and P. M. Platzman, 1991, Phys. Rev. B 43, 6466.

Massoumi, G. R., N. Hozhabri, K. O. Jensen, W. N. Lennard, M. S. Lorenzo, P. J. Schultz, and A. B. Walker, 1992, Phys. Rev. Lett. 68, 3873.

Massoumi, G. R., N. Hozhabri, W. N. Lennard, and P. J. Schultz, 1991, Phys. Rev. B 44, 3486.

Massoumi, G. R., W. N. Lennard, P. J. Schultz, A. B. Walker, and K. O. Jensen, 1993, Phys. Rev. B 47, 11007.

Mattheiss, L. F., 1964, Phys. Rev. 133, A1399.

McMullen, T., 1977, J. Phys. F 7, 2041.

McMullen, T., 1978, J. Phys. F 8, 87.

McMullen, T., 1985, in ICPA85, p. 657.

McMullen, T., 1990, Phys. Rev. B 41, 877.

McMullen, T., P. Jena, S. N. Khanna, Y. Li, and K. O. Jensen, 1991, Phys. Rev. B 43, 10422.

McMullen, T., and M. J. Stott, 1986, Phys. Rev. B 34, 8985.

Mehl, D., A. R. Köymen, K. O. Jensen, F. Gotwald, and A. Weiss, 1990, Phys. Rev. B 41, 799.

Methfessel, M., C. O. Rodriguez, and O. K. Andersen, 1989, Phys. Rev. B 40, 2009.

Mijnarends, P. E., and R. M. Singru, 1979, Phys. Rev. B 19, 6038.

Mikeska, H. J., 1967, Phys. Lett. A 24, 402.

Mills, A. P., Jr., in Positron Solid-State Physics, Proceedings of the International School of Physics, Enrico Fermi, 1981, Course LXXXIII, edited by W. Brandt and A. Dupasquier (North-Holland, Amsterdam), p. 432.

Mills, A. P., Jr., and W. S. Crane, 1985, Phys. Rev. A 31, 593.

Mills, A. P., Jr., E. D. Shaw, M. Leventhal, P. M. Platzman, R.

J. Chichester, D. M. Zuckerman, T. Martin, R. Bruinsma, and R. R. Lee, 1991, Phys. Rev. Lett. 66, 735.

Mills, A. P., Jr., and J. R. Wilson, 1982, Phys. Rev. B 26, 490.

Mott, N. F., and H. S. W. Massey, 1987, The Theory of Atomic Collisions, 3rd ed. (Oxford University Press, Oxford), p. 57.

Neilson, D., R. M. Nieminen, and J. Szymański, 1986, Phys.

Rev. B 33, 1567.

Nielsen, B., K. G. Lynn, and Y.-C. Chen, 1986, Phys. Rev. Lett. 
57, 1789.

Nielsen, B., A. van Veen, L. M. Caspers, H. Filius, H. E. Hansen, and K. Petersen, 1982, in Positron Annihilation, Proceedings of the VI International Conference on Positron Annihilation, Arlington, Texas, 1982, edited by P. G. Coleman, S. C. Sharma, and L. M. Diana (North-Holland, Amsterdam), p. 438.

Nieminen, R. M., 1975, J. Phys. C 8, 2077.

Nieminen, R. M., 1983, in Positron Solid State Physics, Proceedings of the International School of Physics Enrico Fermi, 1981, Course LXXXIII, edited by W. Brandt and A. Dupasquier (North-Holland, Amsterdam), p. 359.

Nieminen, R. M., 1989, in ICPA88, p. 60.

Nieminen, R. M., E. Boroński, and L. Lantto, 1985, Phys. Rev. B 32, 1377.

Nieminen, R. M., and J. Laakkonen, 1979, Appl. Phys. 20, 181.

Nieminen, R. M., J. Laakkonen, P. Hautojärvi, and A. Vehanen, 1979, Phys. Rev. B 19, 1397.

Nieminen, R. M., and M. Manninen, 1979, in Positrons in Solids, Topics in Current Physics No. 12, edited by $P$. Hautojärvi (Springer-Verlag, Berlin), p. 145.

Nieminen, R. M., and J. Oliva, 1980, Phys. Rev. B 22, 2226.

Nieminen, R. M., and M. J. Puska, 1983, Phys. Rev. Lett. 50, 281.

Nieminen, R. M., M. J. Puska, and M. Manninen, 1984, Phys.

Rev. Lett. 53, 1298.

Nieminen, R. M., I. Välimaa, M. Manninen, and P. Hautojärvi, 1980, Phys. Rev. A 21, 1677.

Nørskov, J. K., 1982, Phys. Rev. B 26, 2875.

Nyberg, P., P. Hautojärvi, and K. Härkönen, 1989, unpublished.

Oliva, J., 1980, Phys. Rev. B 21, 4909.

Ore, A., 1949, Naturvidenskap Rekke No. 9, Univ. Bergen Årbok.

Pantelides, S. T., 1986, Ed., Deep Centers in Semiconductors (Gordon and Breach, New York).

Paulin, R., 1979, in Positron Annihilation, Proceedings of the 5th International Conference on Positron Annihilation, Lake Yamanaka, Japan, 1979, edited by R. R. Hasiguti and K. Fujiwara (Japan Institute Met., Tokyo, 1982), p. 601.

Penn, D. R., 1987, Phys. Rev. B 35, 482.

Perdew, J. P., and Y. Wang, 1992, Phys. Rev. B 45, 13244.

Perdew, J. P., and A. Zunger, 1981, Phys. Rev. B 23, 5048.

Perkins, A., and J. P. Carbotte, 1970, Phys. Rev. B 1, 101.

Platzman, P. M., and N. Tzoar, 1986, Phys. Rev. B 33, 5900.

Polatoglou, H. M., and M. Methfessel, 1990, Phys. Rev. B 41, 5898.

Puska, M. J., 1989, J. Phys. Condens. Matter 1, 7347.

Puska, M. J., 1991a, J. Phys. Condens. Matter 3, 3455.

Puska, M. J., 1991b, in ICPA91, p. 419.

Puska, M. J., 1992, unpublished.

Puska, M. J., and C. Corbel, 1988, Phys. Rev. B 38, 9874.

Puska, M. J., C. Corbel, and R. M. Nieminen, 1990, Phys. Rev. B 41, 9980.

Puska, M. J., O. Jepsen, O. Gunnarsson, and R. M. Nieminen, 1986, Phys. Rev. B 34, 2695.

Puska, M. J., T. Korhonen, M. Šob, and G. Brauer, 1994, Phys. Rev. B (in press).

Puska, M. J., P. Lanki, and R. M. Nieminen, 1989, J. Phys. Condens. Matter 1, 6081.

Puska, M. J., S. Mäkinen, M. Manninen, and R. M. Nieminen, 1989, Phys. Rev. B 39, 7666.

Puska, M. J., and M. Manninen, 1987, J. Phys. F 17, 2235.

Puska, M. J., and R. M. Nieminen, 1982, J. Phys. F 12, L123.
Puska, M. J., and R. M. Nieminen, 1983a, J. Phys. F 13, 333. Puska, M. J., and R. M. Nieminen, 1983b, Phys. Scr. T4, 79. Puska, M. J., and R. M. Nieminen, 1984, Phys. Rev. B 39, 5382. Puska, M. J., and R. M. Nieminen, 1992a, Phys. Rev. B 46, 1278.

Puska, M. J., and R. M. Nieminen, 1992b, J. Phys. Condens. Matter 4, L149.

Puska, M. J., R. M. Nieminen, and M. Manninen, 1981, Phys. Rev. B 24, 3037.

Rajainmäki, H., S. Linderoth, H. E. Hansen, and R. M. Nieminen, 1988, J. Phys. F 18, 1109.

Ridley, B. K., 1988, Quantum Processes in Semiconductors (Clarendon, Oxford).

Ritley, K. A., K. G. Lynn, V. J. Ghosh, D. O. Welch, and M. McKeown, 1993, J. Appl. Phys. 74, 3479.

Ritley, K. A., M. McKeown, and K. G. Lynn, 1990, in SLO90, p. 3.

Robertson, I. J., M. C. Payne, and V. Heine, 1991, J. Phys. C 3, 8351.

Rosen, A., D. E. Ellis, H. Adachi, and F. W. Averill, 1976, J. Chem. Phys. 65, 3629.

Rubaszek, A., 1989, J. Phys. Condens. Matter 1, 2141.

Rubaszek, A., 1991, Phys. Rev. B 44, 10857.

Rubaszek, A., A. Kiejna, and S. Daniuk, 1993, J. Phys. Condens. Matter 5, 8195.

Rytsölä, K., K. Rantapuska, and P. Hautojärvi, 1984, J. Phys. B 17, 299.

Saarinen, K., C. Corbel, P. Hautojärvi, P. Lanki, F. Pierre, and D. Vignaud, 1990, J. Phys. Condens. Matter 2, 2453.

Saarinen, K., P. Hautojärvi, P. Lanki, and C. Corbel, 1991, Phys. Rev. B 44, 10585.

Saarinen, K., S. Kuisma, P. Hautojärvi, C. Corbel, and C. LeBerre, 1993, Phys. Rev. Lett. 70, 2794.

Saarinen, K., S. Kuisma, P. Hautojärvi, C. Corbel, and C. LeBerre, 1994, Phys. Rev. B 49, 8005.

Saito, M., A. Oshiyama, and S. Tanigawa, 1991, Phys. Rev. B 44, 10601.

Samara, G. A., 1988, Phys. Rev. B 37, 8523.

Samara, G. A., 1989, Phys. Rev. B 39, 12764.

Schaefer, H. E., M. Forster, R. Würschum, W. Krätschmer, K.

Fostiropoulos, and D. R. Huffman, 1992a, in ICPA91, p. 815.

Schaefer, H. E., M. Forster, R. Würschum, W. Krätschmer, and D. R. Huffman, 1992b, Phys. Rev. B 45, 12164.

Scheffler, M., J. P. Vigneron, and G. B. Bachelet, 1985, Phys. Rev. B 31, 6541.

Schödlbauer, D., P. Sperr, G. Kögel, and W. Triftshäuser, 1988, Nucl. Instrum. Methods B 34, 258.

Schrader, D. M., 1979, Phys. Rev. B 20, 918.

Schultz, P. J., and K. G. Lynn, 1988, Rev. Mod. Phys. 60, 701.

Schultz, P. J., E. Tandberg, B. Nielsen, T. E. Jackman, M. W. Denhoff, and G. C. Aers, 1988, Phys. Rev. Lett. 61, 187.

Seeger, A., F. Banhart, and W. Bauer, 1989, in ICPA88, p. 275.

Singh, D. J., 1993, Physica C 212, 228.

Singh, D., W. E. Picket, R. E. Cohen, H. Krakauer, and S. Berko, 1989, Phys. Rev. B 39, 9667.

Singh, D., W. E. Pickett, E. C. von Stetten, and S. Berko, 1990, Phys. Rev. B 42, 2696.

Skriver, H. L., 1984, The LMTO Method (Springer, New York).

Skriver, H. L., and N. M. Rosengaard, 1992, Phys. Rev. B 45, 9410.

SLO90, 1990, Positron Beams for Solids and Surfaces, AIP Conference Proceedings 218 , edited by P. J. Schultz, G. R. Massoumi, and P. J. Simpson (American Institute of Physics, New York). 
SLO92, 1992, Positron Beams for Solids and Surfaces, edited by A. Weiss, E. Ottewitte, and R. M. Nieminen (unpublished).

Smedskjaer, L. C., A. Bansil, U. Welp, Y. Fang, and K. G. Bailey, 1991, J. Phys. Chem. Solids 52, 1541.

Smedskjaer, L. C., A. Bansil, U. Welp, Y. Fang, and K. G. Bailey, 1992, Physica C 192, 259.

Smedskjaer, L. C., M. Manninen, and M. J. Fluss, 1980, J. Phys. F 10, 2237.

Smedskjaer, L. C., B. W. Veal, D. L. Legnini, A. P. Paulikas, and L. J. Nowicki, 1988, Phys. Rev. B 37, 2330.

SOFTWARE, 1993. Some computer codes of positron physics are available through public-domain files. To obtain information, send e-mail to cmp-soft @ sci.funet.fi.

Soininen, E., D. Beyer, P. Hautojärvi, and J. Mäkinen, 1992, Phys. Rev. B 46, 12394.

Soininen, E., H. Huomo, P. A. Huttunen, J. Mäkinen, P. Hautojärvi, and A. Vehanen, 1990, Phys. Rev. B 41, 6227.

Sorman, H., 1991, Phys. Rev. B 43, 8841.

Sorman, H., and M. Šob, 1990, Phys. Rev. B 41, 10529.

Steindl, R., G. Kögel, P. Sperr, P. Willutzki, D. T. Britton, and W. Triftshäuser, 1992, in ICPA91, p. 1455.

Sterne, P. A., and J. H. Kaiser, 1991, Phys. Rev. B 43, 13892.

Stott, M. J., and P. Kubica, 1975, Phys. Rev. B 11, 1.

Stott, M. J., and R. N. West, 1978, J. Phys. F 8, 635.

Stott, M. J., and E. Zaremba, 1982, Can. J. Phys. 60, 1145.

Sundar, C. S., A. Bharathi, W. Y. Ching, Y. C. Jean, P. H. Hor, R. L. Meng, Z. J. Huang, and C. W. Chu, 1990, Phys. Rev. B 42, 2193.

Tang, C. O., B. R. Li, and A. Chen, 1990, Phys. Rev. B 42, 8078.

Trumpy, G., and M. D. Bentzon, 1992, J. Phys. Condens. Matter 4, 419.

Tuomisaari, M., K. Rytsölä, R. M. Nieminen, and P. Hautojärvi, 1986, J. Phys. B 19, 2667.
Umrigar, C., and D. E. Ellis, 1980, Phys. Rev. B 21, 852.

Valkealahti, S., and R. M. Nieminen, 1983, Appl. Phys. A 32, 95.

Valkealahti, S., and R. M. Nieminen, 1984, Appl. Phys. A 35, 51.

Van House, van Veen, A., H. Schut, J. de Vries, R. A. Hakvoort, and M. R. IJpma, 1990, in SLO90, p. 171.

Vehanen, A., P. Hautojärvi, J. Johansson, and J. Yli-Kauppila, 1982, Phys. Rev. B 25, 762.

Vehanen, A., K. Saarinen, P. Hautojärvi, and H. Huomo, 1987, Phys. Rev. B 35, 4606.

Walker, A. B., K. O. Jensen, J. Szymański, and D. Neilson, 1992, Phys. Rev. B 46, 1687.

Wang, C. Z., C. T. Chang, and K. M. Ho, 1991, Phys. Rev. Lett. 66, 189.

Wang, X.-G., and H. Zhang, 1990, J. Phys. Condens. Matter 2, 7275.

Weinert, M., and R. E. Watson, 1984, Phys. Rev. B 29, 3001.

Weiss, A., R. Mayer, M. Jibaly, C. Lei, D. Mehl, and K. G. Lynn, 1988, Phys. Rev. Lett. 61, 2245.

West, R., 1973, Adv. Phys. 22, 263.

West, R., 1979, in Positrons in Solids, Topics in Current Physics No. 12, edited by P. Hautojärvi (Springer-Verlag, Berlin), p. 89.

Wilson, R. J., 1983, Phys. Rev. B 27, 6974.

Wilson, R. J., and A. P. Mills, Jr., 1983, Surf. Sci. 128, 70.

Wilson, W. D., C. L. Bisson, and M. I. Baskes, 1981, Phys. Rev. B 24, 5616.

Woll, E. J., and J. P. Carbotte, 1967, Phys. Rev. 164, 985.

Xu, H., 1990, Phys. Rev. B 42, 11295.

Xu, H., and U. Lindefelt, 1990, Phys. Rev. B 41, 5979.

Zhang, S. B., and J. E. Northrup, 1991, Phys. Rev. Lett. 67, 2339. 


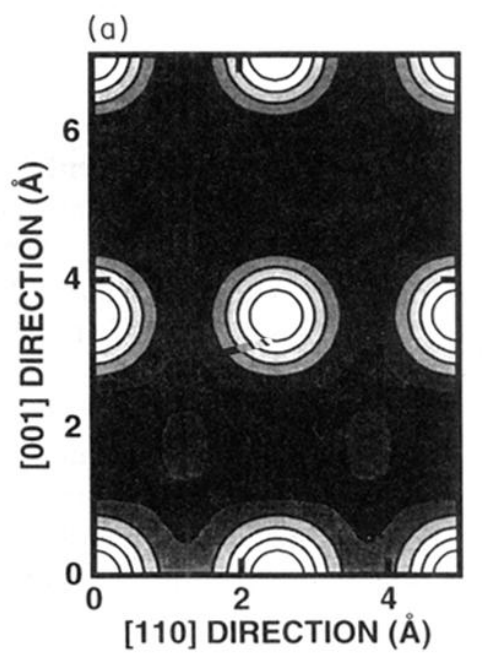

(c) GRAPHITE

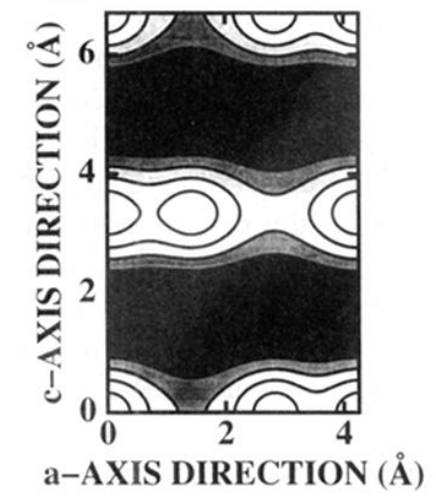

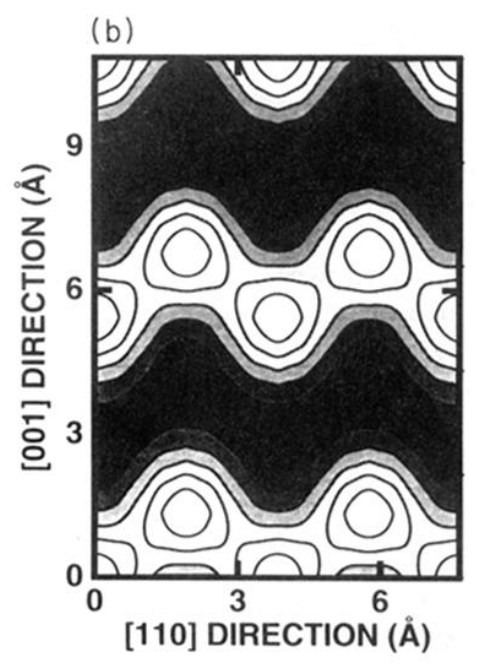

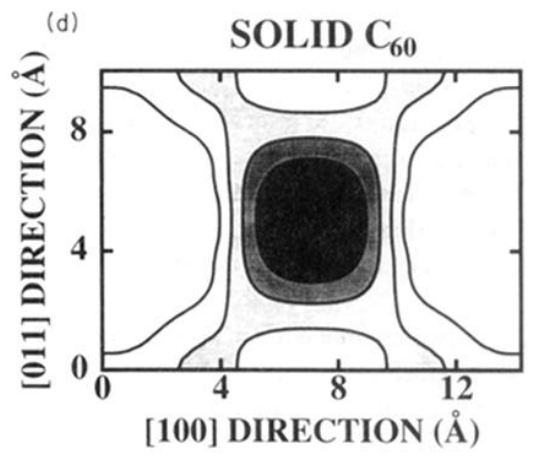

FIG. 1. Positron wave functions in perfect solids: (a) fcc Ni; (b) Si with diamond structure; (c) graphite; (d) solid $\mathrm{C}_{60}$. The calculations were performed using the superimposed-atom method (Puska and Nieminen, 1983a). The contour spacing is one-sixth of the maximum value. The wave function is vanishingly small inside the ion-core regions and inside the $\mathrm{C}_{60}$ molecules, which show in white in the figure. The darker shading indicates larger values of the wave function. 

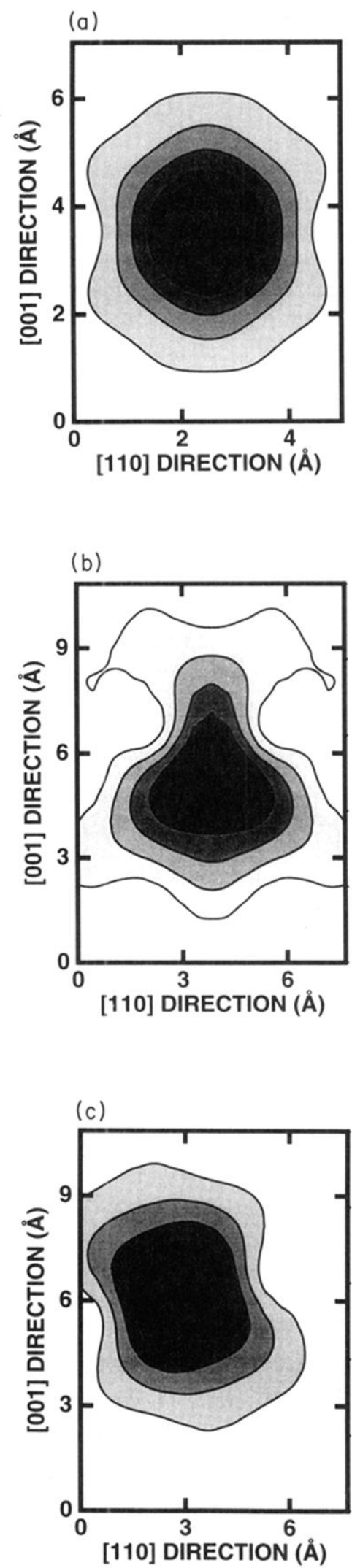

FIG. 2. Positron wave functions for vacancy-type defects in solids: (a) vacancy in fcc $\mathrm{Ni}$; (b) vacancy in $\mathrm{Si}$ with diamond structure; (c) divacancy in $\mathrm{Si}$. The calculations were performed using the superimposed-atom method (Puska and Nieminen, 1983a). See the caption of Fig. 1. The figure planes correspond to those for the perfect crystals in Figs. 1(a) and 1(b). 